\title{
Design, Synthesis, and Biological Evaluation of Phosphinopeptides against Trypanosoma cruzi Targeting Trypanothione Biosynthesis
}

\author{
Esteban L. Ravaschino, ${ }^{\dagger}$ Roberto Docampo, ${ }^{\ddagger}$ and Juan B. Rodriguez ${ }^{\dagger}, *$ \\ Departamento de Química Orgánica, Facultad de Ciencias Exactas y Naturales, Universidad de Buenos Aires, Pabellón 2, Ciudad \\ Universitaria, C1428EHA, Buenos Aires, Argentina, and Center for Global and Emerging Diseases and Department of Cellular Biology, \\ University of Georgia, Athens, Georgia, 30602
}

Received September 16, 2005

As a part of our project aimed at the search for new safe chemotherapeutic and chemoprophylactic agents against American trypanosomiasis (Chagas's disease), a series of phosphinopeptides structurally related to glutathione was designed, synthesized, and evaluated as antiproliferative agents against the parasite responsible for this disease, the hemoflagellated protozoan Trypanosoma cruzi. The rationale for the synthesis of these compounds was supported on the basis that the presence of the phosphinic acid moiety would mimic the tetrahedral transition state of trypanothione synthase (TryS), a typical C:N ligase, and the molecular target of these drugs. Of the designed compounds, $\mathbf{5 3}$ and $\mathbf{5 4}$ were potent growth inhibitors against the clinically more relevant form of $T$. cruzi (amastigotes) growing in myoblasts. The efficacy for these drugs was comparable to that exhibited by the well-known antiparasitic agent WC-9. The simple phosphinopeptide structure found as a pharmacophore in the present study constitutes a starting point for the development of straightforward optimized drugs.

\section{Introduction}

Prospects in antileishmanial and antitrypanosomal drug research have changed substantially since the discovery of trypanothione 1. ${ }^{1}$ The uniqueness of this metabolite and its biosynthetic pathway in parasites of the order Kinetoplastida confer to the involved enzymes of its biosynthesis a great usefulness as a molecular target. Current chemotherapy is based on empirically discovered drugs that interact with parasitic metabolic pathways that have their corresponding counterparts in mammals; therefore, the efficacy of these drugs is sustained by the ability of the host to balance metabolic deficiencies with alternate pathways. In addition, trypanothione is essential for parasite survival for two reasons: (a) its crucial role in regulating the cellular redox equilibrium, (b) pathogenic trypanosomes do not have an alternate mechanism to protect against the oxidative stress. ${ }^{2}$ Trypanothione $\left(N^{1}, N^{8}\right.$-bis(glutathionyl)spermidine) is the bisconjugated product between the tripeptide glutathione ( 2 , $\mathrm{GSH})$ and the polyamine spermidine $(\mathbf{3}, \mathrm{Spd})$. The cysteine residues present in the trypanothione moiety are involved in the interconversion between its oxidized form $\left[\mathbf{1 a}, \mathrm{T}(\mathrm{S})_{2}\right]$ and reduced form $\left[\mathbf{1 b}, \mathrm{T}(\mathrm{SH})_{2}\right]$ by cleaving and restoring an intramolecular disulfide bridge. Both structures have a +1 net charge at physiologic $\mathrm{pH}$ (Scheme 1$){ }^{3}$

The enzyme responsible for the reduction of trypanothione was isolated and named trypanothione reductase. ${ }^{4-7}$ This enzyme, which is highly specific for trypanothione, does not recognize glutathione as a substrate.

Trypanosomatids, as other aerobic organisms, are exposed to highly reactive oxygen species such as $\mathrm{O}_{2}{ }^{-}, \mathrm{H}_{2} \mathrm{O}_{2}$, and $\mathrm{HO}$. $\mathrm{HO} \bullet$, and $\mathrm{RO} \bullet$ radicals can cause lethal damage if irreversibly react with DNA or cellular membrane lipids. ${ }^{8}$ To avoid this problem, the defense system maintains low levels of $\mathrm{H}_{2} \mathrm{O}_{2}$ and

* To whom correspondence should be addressed. E-mail: jbr@qo.fcen.uba.ar.

† Universidad de Buenos Aires.

$\doteqdot$ University of Georgia.
Scheme 1. Oxidized and Reduced Form of Trypanothione (compounds $\mathbf{1 a}$ and $\mathbf{1 b}$, respectively)

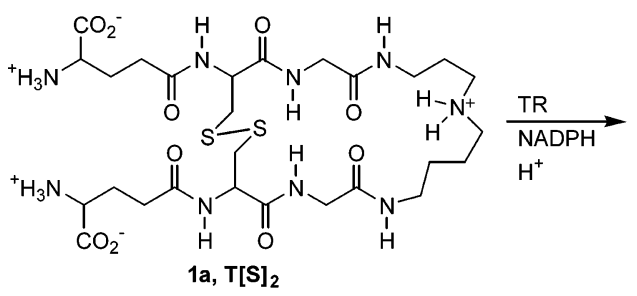

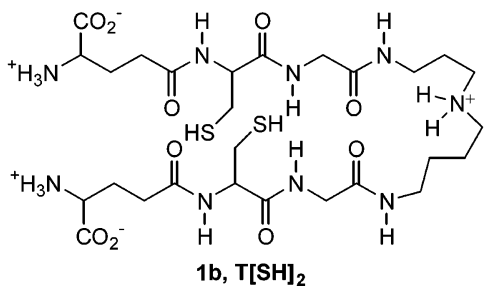

$\mathrm{O}_{2}{ }^{-}$. Superoxide dismutases (SOD) have been identified in $T$. cruzi,${ }^{9}$ Leishmania donovani, ${ }^{10}$ and C. fasciculata. Glutathione reductase activity is not found in trypanosomes, but a highly efficient trypanothione reductase activity is present instead. Glutathione $S$-transferases are found in more developed organisms, while typanothione $S$-transferase activity is present in several parasites such as $C$. fasciculata, L. major, L. infantum, L. tarentolae, and T. brucei, associated with the 1B eukaryotic elongation factor (eEF1B). ${ }^{11}$ The eEF1B has been expressed, cloned, and purified in L. major ${ }^{12}$ catalyzing conjugation between a variety of electrophiles with $\mathbf{1}$ and glutathionylspermidine (4) but not with 2 . Once again, this finding suggests that trypanothione plays the same role in parasites that glutathione does in mammals.

The last two steps of trypanothione biosynthesis are the most relevant ones in terms of molecular targets, because they involve enzymes that have no counterpart in mammals. These flavoenzymes are ATP-dependent $\mathrm{C}: \mathrm{N}$ ligases, which require $\mathrm{Mg}^{2+}$ as cofactor, catalyze the conjugation of $\mathbf{3}$ with two molecules of $\mathbf{2}$ 
Scheme 2. Two Possibilities for Trypanothione Biosynthesis

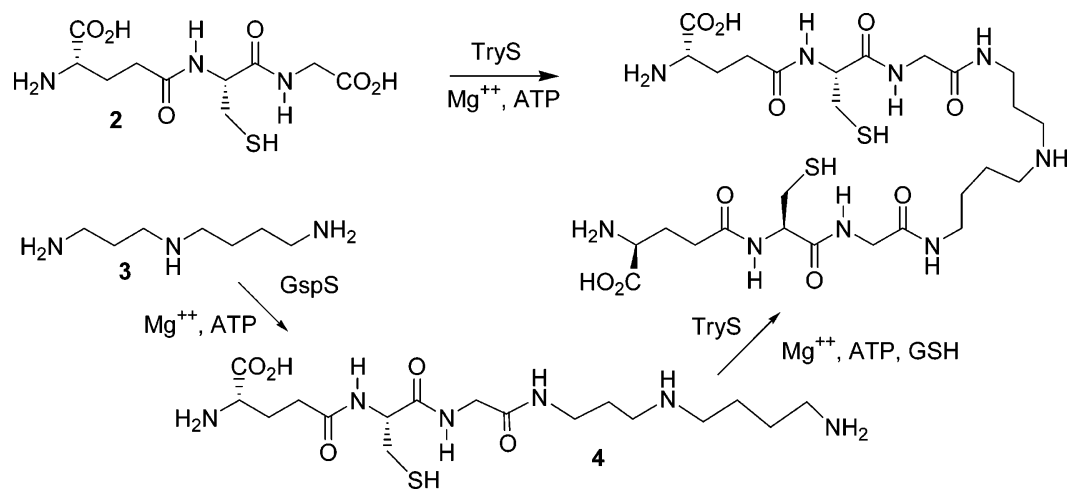

to yield 1. At the present time is not completely clear how this conjugation undergoes in all parasites of the order Kinetoplastida. Two possibilities are being investigated: one comprises two distinct enzymes (GspS and TryS) that catalyze sequentially the conjugation of $\mathbf{2}$ with $\mathbf{3}$ to form $\mathbf{4}$, and the reaction of this resulting product with another molecule of $\mathbf{2}$; the other one involves only one enzyme (TryS), which is able to catalyze biosynthesis of $\mathbf{1}$ directly from $\mathbf{2}$ and $\mathbf{3}$ (Scheme 2). ${ }^{13-26}$

Prospects in searching molecular targets began to change substantially when $T$. cruzi TryS was cloned and expressed in E. coli. ${ }^{27}$ This protein was not only able to synthesize trypanothione from glutathionylspermidine and glutathione but also from spermidine and glutathione. At this point, no gene encoding a GspS could be identified in $T$. cruzi, so it was postulated that in this parasite a single enzyme catalyzed formation of trypanothione. ${ }^{27}$ The dual catalytic function of T.cruzi TryS, also found in $T$. brucei brucei, ${ }^{28}$ led to investigate C. fasciculata TryS catalytic properties. The enzyme was cloned and expressed in E. coli as an active protein that catalyzed trypanothione formation starting either from glutathionylspermidine and GSH or from spermidine and GSH. ${ }^{29}$ In addition, Leishmania major TryS was cloned and expressed in E. coli. ${ }^{30}$ On the other hand, a pseudogene that encodes GspS (GSPS) was identified in this parasite. This last finding suggests that an evolutionary link occurs to resolve the observed divergence between $C$. fasciculata and Trypanosoma spp. ${ }^{30}$ It seems reasonable to hypothesize that an ancestral GSPS with a narrow substrate specificity may have undergone duplication and divergence into two independent genes (GSPS and TRYS). This is the case of the substrate-specific enzymes (GspS and TryS) found in C. fasciculata. Trypanosoma genome had only retained TRYS; therefore, TryS evolved into a broad-specificity enzyme superseding the need for two enzymes in trypanothione biosynthesis. ${ }^{30,31}$

\section{Rationale}

As trypanosomatids possess a strict requirement for trypanothione for survival and growth that cannot be replaced by glutathione present in the mammalian host, depletion of this metabolite will lead either to cell death or to growth impairment. Bearing in mind that a rather similar mechanism of cell protection is present in several pathogenic trypanosomes such as $T$. cruzi, T. brucei rhodesiense, and T. brucei gambiense, and Leishmania spp., this approach can be useful for the treatment of different trypanosomiases and leishmaniasis. Since this metabolic pathway is absent in the host, it is possible to envisage that a highly selective inhibition of the enzymatic activity either of TryS or GspS would not have any toxic effect against mammalian cells. The crystal structure of TryR is now available,${ }^{32-34}$ while the corresponding X-ray structure for GspS and TryS have not been resolved yet. The lack of information of the three-dimensional structure of the target proteins have circumscribed the search of inhibitors of the enzymatic activity against these enzymes to the classical chemical structure/ biological activity relationship (SAR). Therefore, the design of inhibitors of the enzymatic activity for the mentioned ligases should be based on isosteric replacements on the substrate or on analogues of the transition state. The inhibition of the enzymatic activity of these enzymes has been studied and some data dealing with inhibitors of GSS activity are available. For example, it has been found that analogues of glutathione in which isoleucine replaced the cysteine residue to form $\mathrm{L}-\gamma$-GluL-Ile-Gly (5) or L- $\gamma$-Glu-L-Ile-L-Ala (6) exhibited noncompetitive inhibition with $K_{\mathrm{i}}$ in the low millimolar range. ${ }^{35}$ Phosphonates and phosphinates are well-known inhibitors of C:N-ligases. ${ }^{36,37}$ These findings have led to the design of phosphorus-containing drugs that behave as inhibitors of the enzymatic activity of GspS. For example, 7 exhibits linear noncompetitive inhibition with a $K_{\mathrm{i}}$ value of $60 \mu \mathrm{M}$ against $\mathrm{GspS}$ from C. fasciculata. $^{38}$ Moreover, when compounds structurally related to 7 are coupled with spermidine, slow tight-binding inhibitors of GspS were obtained. ${ }^{39,40}$ For instance, 8 exhibits a $K_{\mathrm{i}}$ value of $3.2 \mu \mathrm{M}$ for binding to free enzyme (GspS from Escherichia coli) and 7.8 $\mathrm{nM}$ for binding to enzyme-substrate complex $\mathrm{E} \cdot \mathrm{I}^{*}$, while 9 was much less potent than 7 showing inhibition constants of 6 $\mu \mathrm{M}$ and $14 \mu \mathrm{M}$ for binding free enzyme and $\mathrm{E} \cdot \mathrm{I}^{*}$, respectively. ${ }^{39,40}$ These compounds would act as inhibitors of the transition state at the active site of GspS.
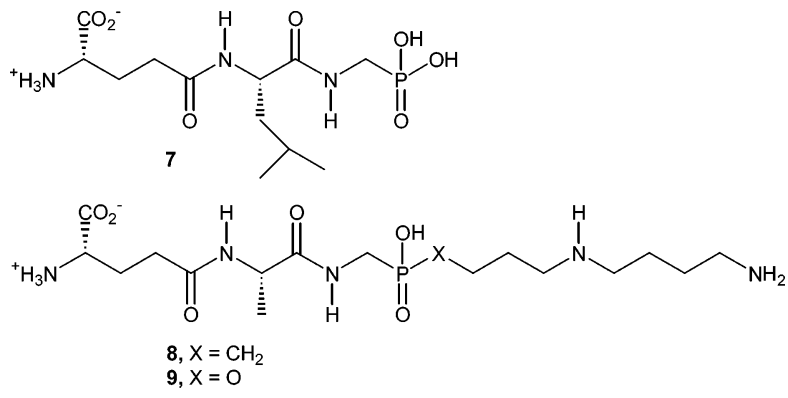

We have described a new series of analogues of glutathione (L- $\gamma$-Glu-L-Leu-Gly), where the glycine carboxylic acid group has been substituted by other acidic groups such as tetrazole, hydroxamic acid, acylsulfonamide, and boronic acid. Among this new family of compounds, the boronic acid derivative $\mathbf{1 0}$ is an effective inhibitor of GspS with a $K_{\mathrm{i}}$ value of $81 \mu \mathrm{M}$ and an $\mathrm{IC}_{50}$ value of $17 \mu \mathrm{M} .^{41}$ In addition, substitution of the glycine part on the same tripeptide leads to good inhibitors, specifically when the glycine moiety is replaced by diaminopropionic acid 
Scheme $3^{a}$

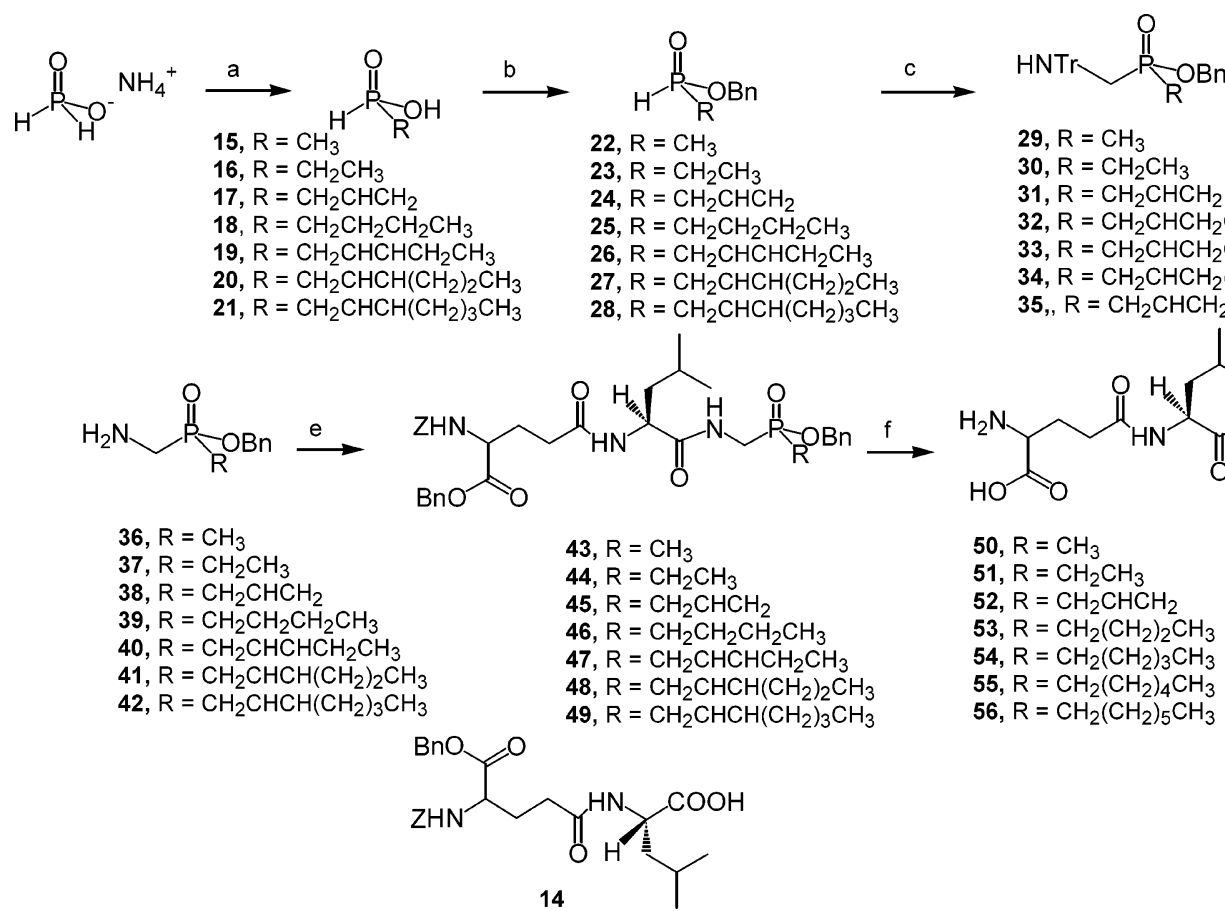

${ }^{a}$ Reagents and conditions: (a) (i) $\mathrm{H}_{2} \mathrm{PO}_{2}-\mathrm{NH}_{4}{ }^{+}$, HMDS, $110{ }^{\circ} \mathrm{C}, 2 \mathrm{~h}$, (ii) $\mathrm{RI}, \mathrm{CH}_{2} \mathrm{Cl}_{2}, \mathrm{rt}, 16 \mathrm{~h}$; (b) $\mathrm{BnOH}, \mathrm{DCC}, \mathrm{DMAP}, \mathrm{THF}, \mathrm{rt}, 16 \mathrm{~h}$; (c) $\mathrm{TrN}=\mathrm{CH}_{2}$, $\mathrm{BF}_{3} \cdot \mathrm{Et}_{2} \mathrm{O}, \mathrm{MePh}, \mathrm{rt}, 3 \mathrm{~d}$; (d) TFA (2.5\%), $\mathrm{CH}_{2} \mathrm{Cl}_{2}, \mathrm{rt}, 30 \mathrm{~min}$; (e) (i) $N$-hydroxysuccinimide, EDC $\cdot \mathrm{HCl}, \mathrm{DMF}, \mathrm{rt}, 16 \mathrm{~h}$, (ii) $\mathrm{Et} 3 \mathrm{~N}, \mathrm{DMF}, \mathrm{rt}, 16 \mathrm{~h}$; (f) $\mathrm{H}_{2}, \mathrm{Pd} / \mathrm{C}$, $\mathrm{MeOH}, 1 \mathrm{~atm}, \mathrm{rt}, 1 \mathrm{~h}$.

to form 11. This compound was a potent inhibitor of GspS activity with $K_{\mathrm{i}}=7.2 \mu \mathrm{M} .{ }^{42}$<smiles>[2H]N(CB(O)O)C(=O)[C@@H](CC(C)C)N([2H])C(=O)CC[C@H](N)C(=O)O</smiles>

In addition, D'Silva et al. ${ }^{43,44}$ have reported the biological activity of glutathione derivatives targeting trypanothione metabolism, where the protected tripeptide $\mathbf{1 2}$ arises as main member of this class of gluthation analogues. This compound is moderately potent against $T$. brucei $\left(\mathrm{ED}_{50}=1.9 \mu \mathrm{M}\right)$ but exhibits very weak biological activity against $T$. cruzi. $^{43,44}$ The above results encouraged us to prepare analogues assuming that the optimum glutathione tripeptide derivative should be $\mathrm{L}-\gamma$ Glu-L-Leu-Gly. Unexpectedly, $N$-alkyl amide derivatives of gluthatione of general formula $\mathbf{1 3}$ are devoid of activity against T. cruzi growth. ${ }^{45}$ The lack of biological action of $\mathbf{1 3}$ might be attributable to the amidase activity of the target enzyme. This enzyme, despite being unique for trypanosomatids with a specific function of catalyzing the first of the two ultimate steps of trypanothione biosynthesis, would act as a bifunctional synthetase/amidase. ${ }^{46,47}$ The loss of the $\mathrm{N}$-alkylamine moiety would be responsible for the marginal activity observed. A similar approach for glutathione biosynthesis inhibition was depicted employing buthionine sulfoxime as substrate analogue. ${ }^{48}$

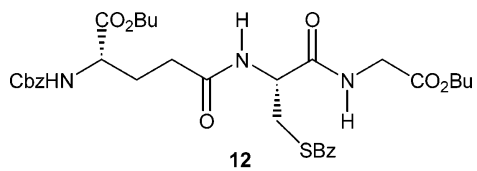<smiles>[R]NC(=O)CNC(=O)[C@H](CC(C)C)NC(=O)CC[C@H](N)C(=O)O</smiles>

\section{Results and Discussion}

Compound 8 and 9 represent interesting examples of slow, tight-binding inhibitors of the enzymatic activity of GspS. These compounds act by mimicking the transition state for amide bond formation of this $\mathrm{C}: \mathrm{N}$ ligase. However, any attempt to optimize the biological action of these compounds would be impractical from the synthetic point of view. In fact, the chemical synthesis of these compounds, or structurally related analogues, requires many synthetic steps due to the complexity of their structures. In the search for a simpler pharmacophore than $\mathbf{8}$, keeping this structure as lead drug, a series of phosphinopeptide analogues 50-56 was prepared. The peptidic moiety was modified by replacing the L-cysteine residue by L-leucine on the basis that this replacement converts an enzyme substrate into an inhibitor of the enzymatic activity of the same enzyme. ${ }^{38}$ The phosphinate unit was retained, while the polyamine residue was changed to an $n$-alkyl group. The preparation of the designed compounds was carried out starting from ammonium hypophosphite, which on reaction with hexamethyldisilazane at $120{ }^{\circ} \mathrm{C}$ for $2 \mathrm{~h},{ }^{49}$ followed by alkylation with the appropriate alkyl iodide, and further esterification with benzylic alcohol in the presence of dicyclohexylurea and 4-(dimethylamino)pyridine was converted into the corresponding benzyl alkylphosphinate (compounds 22-28) (Scheme 3). The P-alkylation reaction occurred smoothly if the alkylating agent had up to four carbon atoms. The 
Table 1. Effect of Phosphinopeptides against Trypanosoma cruzi (amastigotes and epimastigotes) for Compounds 50-56

\begin{tabular}{clc}
\hline compd & $\begin{array}{c}\text { amastigotes: } \\
\mathrm{IC}_{50}(\mu \mathrm{M})^{a}\end{array}$ & $\begin{array}{c}\text { epimastigotes: } \\
\mathrm{IC}_{50}(\mu \mathrm{M})^{a}\end{array}$ \\
\hline $\mathbf{5 0}$ & $>50$ & $>50$ \\
$\mathbf{5 1}$ & $>50$ & $>50$ \\
$\mathbf{5 2}$ & $>50$ & $>50$ \\
$\mathbf{5 3}$ & 9.8 & $>50$ \\
$\mathbf{5 4}$ & 14.2 & $>50$ \\
$\mathbf{5 5}$ & $>50(45 \%)^{b}$ & $>50$ \\
$\mathbf{5 6}$ & $>50(31 \%)^{b}$ & $>50$ \\
$\mathbf{5 7}$ & 2.0 &
\end{tabular}

${ }^{a}$ Values are means of three experiments. ${ }^{b}$ Maximum inhibition values obtained at the indicated concentrations are given in parentheses.

preparation of alkyl derivatives of more than four carbon atoms required more reactive electrophiles such as 2-alkenyl iodides, which were reduced to the corresponding saturated $n$-alkyl derivatives, together with protecting groups, removed by catalytic hydrogenation during the last synthetic step. The resulting $O$-benzyl alkylphosphinates treated with methylene tritylamine and boron trifluoride etherate in toluene afforded the respective $O$-benzyl alkyl [( $N$-tritylamine $)$ methyl]phosphinate (compounds 29-35). Removal of the trityl protecting group yielded the corresponding $O$-benzyl alkyl(aminomethylmethyl)phosphinates (compounds 36-42) that was conjugated with dipeptide $\mathbf{1 4}$ to give the immediate precursor of the title compounds (43-49). Protecting group cleavage by catalytic hydrogenation afforded the desired compounds $(\mathbf{5 0 - 5 6 )}$ that were evaluated against the clinically more relevant form of the parasite (the intracellular amastigotes) and the dividing noninfective epimastigote forms of the parasite. Of the designed compounds, $\mathbf{5 3}$ and $\mathbf{5 4}$ were potent inhibitors against intracellular $T$. cruzi proliferation possessing $\mathrm{IC}_{50}$ values of $9.8 \mu \mathrm{M}$ and $14.2 \mu \mathrm{M}$, respectively. The inhibitory efficacy of these compounds was comparable to the potency exhibited by 4-phenoxyphenoxyethyl thiocyanate 57 (WC-9), ${ }^{50-53}$ a wellknown antiparasitic agent, which was used as a positive control. Derivatives with short aliphatic chains were not active against amastigotes, while $\mathbf{5 5}$ and $\mathbf{5 6}$ were moderately potent against this form of the parasite. All of these compounds were devoid of activity against the noninfective epimastigote forms of the parasite at concentrations up to $50 \mu \mathrm{M}$. The inhibition data are illustrated in Table 1. This particular behavior has also been observed in other antiparasitic agents with a marked different mode of action such as bisphosphonates, which are very potent growth inhibitors of the amastigote forms of $T$. cruzi but have very weak action toward epimastigotes. ${ }^{54,55}$

These compounds, at concentrations up to $50 \mu \mathrm{M}$, exhibited no toxicity to the host cells, as assessed by phase contrast microscopy observation of detachment, vacuolation, and rounding of the cells. In conclusion, we found a simple pharmacophore structure that can be straightfowardly prepared from inexpensive starting materials involving few synthetic steps. This simple pharmacophore prompts new approaches in the field of tropical diseases chemotherapy. Compound $\mathbf{5 3}$ is an encouraging model of an effective antiparasitic agent, which presents potential prospects as a pharmacophore to be used for further structure optimization. It has two well-defined parts: (a) a peptidic moiety, and (b) an aliphatic unit. Both of these parts of the molecule can be easily modified. Metabolic stability, cell penetration, bioavailavity, etc., are common problems associated with peptide analogues. Therefore, the design of peptidomimetics that retain the pharmacophoric unit would provide metabolically stable analogues with similar antiparatic activities. Efforts in these aspects are currently being pursued in our laboratory.

\section{Experimental Section}

The glassware used in air and/or moisture sensitive reactions was flame-dried and carried out under a dry argon atmosphere. Unless otherwise noted, chemicals were commercially available and used without further purification. Solvents were distilled before use. Tetrahydrofuran and toluene were distilled from sodium/benzophenone ketyl. Anhydrous $N, N$-dimethylformamide was used as supplied from Aldrich.

Nuclear magnetic resonance spectra were recorded using a Bruker AC-200 MHz or a Bruker AM-500 MHz spectrometers. Chemical shifts are reported in parts per million $(\delta)$ relative to tetramethylsilane. Coupling constants are reported in Hertz. ${ }^{13} \mathrm{C}$ NMR spectra were fully decoupled. Splitting patterns are designated as s, singlet; d, doublet; t, triplet; q, quartet.

High-resolution mass spectra was conducted using a Micromass Q-Tof Ultima apparatus, which is a hybrid quadrupole time-offlight mass spectrometer with MS/MS capability.

Melting points were determined using a Fisher-Johns apparatus and are uncorrected. IR spectra were recorded using a Nicolet Magna 550 spectrometer.

Column chromatography was performed with E. Merck silica gel (Kieselgel 60, 230-400 mesh). Analytical thin-layer chromatography was performed employing $0.2 \mathrm{~mm}$ coated commercial silica gel plates (E. Merck, DC-Aluminum sheets, Kieselgel 60 $\mathrm{F}_{254}$ ).

General Procedure for the One-Pot Preparation of Phosphinic Acid Benzyl Ester. A mixture of ammonium phosphinate $(1.00 \mathrm{~g}, 12.04 \mathrm{mmol})$ and hexamethyldisilazane $(2.53 \mathrm{~mL}, 12.04$ mmol) under an argon atmosphere was stirred at $110{ }^{\circ} \mathrm{C}$ for $2 \mathrm{~h}$. The reaction mixture was cooled at $0{ }^{\circ} \mathrm{C}$ and anhydrous methylene chloride $(12 \mathrm{~mL})$ added, followed by addition of the corresponding alkyl halide (12.04 mmol). The mixture was allowed to reach room temperature and stirred overnight. A yellow precipitate appeared, and the mixture was filtered through Celite under nitrogen. Anhydrous benzyl alcohol $(2.46 \mathrm{~mL}, 24.08 \mathrm{mmol})$ was added to the filtrate and the solvent removed at room temperature under reduced pressure. Pressure was restored with argon. Anhydrous tetrahydrofuran $(20 \mathrm{~mL})$ was added followed by addition of an additional equivalent of benzyl alcohol $(1.23 \mathrm{~mL}, 12.04 \mathrm{mmol})$, 4-(dimethylamino)pyridine $(100 \mathrm{mg})$, and dicyclohexylcarbodiimide (3.23 g, $15.65 \mathrm{mmol})$ at $0{ }^{\circ} \mathrm{C}$. The reaction mixture was stirred at room-temperature overnight. White dicyclohexylurea precipitated, and the suspension was filtered through a glass frit filter. The solvent was evaporated and the residue purified by column chromatography (silica gel) employing mixtures of $\mathrm{CH}_{2} \mathrm{Cl}_{2}$-methanol ranging from 100:0 to 97:3. The oil obtained was redissolved in a minimum of a mixture of hexanes-EtAcO (1:1) and filtered through Celite to eliminate the remaining dicyclohexylurea. The solvent was evaporated, and the purified compound was immediately used in next step reaction before hydrolysis could take place. Reaction yields were based on ammonium phosphinate.

Methylphosphinic Acid Benzyl Ester (22). Colorless oil, 46\% yield; ${ }^{1} \mathrm{H}$ NMR $\left(500.13 \mathrm{MHz}, \mathrm{CD}_{3} \mathrm{OD}\right) \delta 1.47\left(\mathrm{dd}, J_{\mathrm{P}-\mathrm{H}}=15.3\right.$ $\left.\mathrm{Hz}, J_{\mathrm{H}-\mathrm{H}}=2.1 \mathrm{~Hz}, 3 \mathrm{H}, \mathrm{PCH} \mathrm{H}_{3}\right), 4.59\left(2 \mathrm{H}, \mathrm{OCH}_{2} \mathrm{Ph}\right), 7.13\left(\mathrm{~d}, J_{\mathrm{P}-\mathrm{H}}\right.$ $=546.8 \mathrm{~Hz}, \mathrm{PH}), 7.21-7.25(\mathrm{~m}, 1 \mathrm{H}, \mathrm{Ph}) ; 7.28-7.36(\mathrm{~m}, 4 \mathrm{H}$, $\mathrm{Ph}) .{ }^{13} \mathrm{C}$ NMR $\left(125.77 \mathrm{MHz}, \mathrm{CD}_{3} \mathrm{OD}\right) \delta 17.0(\mathrm{~d}, J=93.3 \mathrm{~Hz}$, $\left.\mathrm{PCH}_{3}\right), 65.2\left(\mathrm{OCH}_{2} \mathrm{Ph}\right), 128.0(\mathrm{Ph}), 128.2(\mathrm{Ph}), 129.3(\mathrm{Ph}), 142.7$ $(\mathrm{Ph}) ;{ }^{31} \mathrm{P}$ NMR $\left(202.45 \mathrm{MHz}, \mathrm{CD}_{3} \mathrm{OD}\right) \delta 30.1$.

Ethylphosphinic Acid Benzyl Ester (23). Colorless oil, 32\% yield; ${ }^{1} \mathrm{H} \mathrm{NMR}\left(200 \mathrm{MHz}, \mathrm{CDCl}_{3}\right) \delta 1.16\left(\mathrm{~d}, J_{\mathrm{P}-\mathrm{H}}=21.4 \mathrm{~Hz}\right.$, $\left.J_{\mathrm{H}-\mathrm{H}}=7.7 \mathrm{~Hz}, 3 \mathrm{H}, \mathrm{CH}_{3}\right), 1.7-1.95\left(\mathrm{~m}, 2 \mathrm{H}, \mathrm{PCH}_{2} \mathrm{CH}_{3}\right), 5.08(\mathrm{dd}$, $\left.J_{\mathrm{P}-\mathrm{H}}=24.7 \mathrm{~Hz}, J_{\mathrm{H}-\mathrm{H}}=11.7 \mathrm{~Hz}, 1 \mathrm{H}, \mathrm{PC} H_{\mathrm{a}} \mathrm{Ph}\right), 5.12\left(\mathrm{dd}, J_{\mathrm{P}-\mathrm{H}}=\right.$ $\left.23.0 \mathrm{~Hz}, J_{\mathrm{H}-\mathrm{H}}=11.7 \mathrm{~Hz}, 1 \mathrm{H}, \mathrm{PCH} H_{\mathrm{a}} \mathrm{Ph}\right), 7.09\left(\mathrm{dt}, J_{\mathrm{P}-\mathrm{H}}=530.0\right.$ $\left.\mathrm{Hz}, J_{\mathrm{H}-\mathrm{H}}=1.8 \mathrm{~Hz}, 1 \mathrm{H}, H \mathrm{P}\right), 7.27-7.50(\mathrm{~m}, 5 \mathrm{H}, \mathrm{Ph})$.

Allylphosphinic Acid Benzyl Ester (24). Colorless oil, 43\% yield; ${ }^{1} \mathrm{H}$ NMR $\left(200 \mathrm{MHz}, \mathrm{CDCl}_{3}\right) \delta 2.63-2.67\left(\mathrm{~m}, 2 \mathrm{H}, \mathrm{PCH}_{\mathrm{a}-}\right.$ 
$\left.\mathrm{CHCH}_{2}\right), 2.67-2.70\left(\mathrm{~m}, 2 \mathrm{H}, \mathrm{PCH}_{\mathrm{b}} \mathrm{CHCH}_{2}\right), 5.06\left(\mathrm{dd}, J_{\mathrm{H}-\mathrm{H}}=11.8\right.$ $\left.\mathrm{Hz}, J_{\mathrm{P}-\mathrm{H}}=8.7 \mathrm{~Hz}\right), 5.15\left(\mathrm{dd}, J_{\mathrm{H}-\mathrm{H}}=11.8 \mathrm{~Hz}, J_{\mathrm{P}-\mathrm{H}}=10.1 \mathrm{~Hz}\right)$, 5.23 (dddt, $1 \mathrm{H},{ }^{3} J_{\mathrm{H}-\mathrm{H}}=17.1 \mathrm{~Hz}, J_{\mathrm{P}-\mathrm{H}}=6.1 \mathrm{~Hz},{ }^{2} J_{\mathrm{H}-\mathrm{H}}=1.3 \mathrm{~Hz}$, $\left.{ }^{3} J_{\mathrm{H}-\mathrm{H}}=1.4 \mathrm{~Hz}, \mathrm{PCH}_{2} \mathrm{CHCH}_{\mathrm{a}}\right), 5.27$ (dddt, $1 \mathrm{H},{ }^{3} J_{\mathrm{H}-\mathrm{H}}=10.2 \mathrm{~Hz}$, $J_{\mathrm{P}-\mathrm{H}}=5.4 \mathrm{~Hz},{ }^{2} J_{\mathrm{H}-\mathrm{H}}=1.3 \mathrm{~Hz},{ }^{3} J_{\mathrm{H}-\mathrm{H}}=1.1 \mathrm{~Hz}, \mathrm{PCH}_{2} \mathrm{CHCH}_{\mathrm{b}}$ ), $5.73\left(\mathrm{dddt},{ }^{3} J_{\mathrm{H}-\mathrm{H}, \text { trans }}=17.1 \mathrm{~Hz},{ }^{3} J_{\mathrm{H}-\mathrm{H} \text {, cis }}=10.1 \mathrm{~Hz}, J_{\mathrm{P}-\mathrm{H}}=6.9\right.$ $\mathrm{Hz},{ }^{3} J_{\mathrm{H}-\mathrm{H}}=7.5 \mathrm{~Hz}, 1 \mathrm{H}, \mathrm{PCH}_{2} \mathrm{CHCH}_{2}$ ), 7.04 (ddd, $J_{\mathrm{P}-\mathrm{H}}=546.8$ $\left.\mathrm{Hz}, J_{\mathrm{H}-\mathrm{H}}=2.2 \mathrm{~Hz}, J_{\mathrm{H}-\mathrm{H}}=1.8 \mathrm{~Hz}, 1 \mathrm{H}, H \mathrm{P}\right), 7.24-7.31(\mathrm{~m}, 1 \mathrm{H}$, $\mathrm{Ph}), 7.31-7.41(\mathrm{~m}, 4 \mathrm{H}, \mathrm{Ph}) ;{ }^{13} \mathrm{C}$ NMR $\left(125.77 \mathrm{MHz}, \mathrm{CDCl}_{3}\right) \delta$ $35.3\left(\mathrm{~d}, J=90.7 \mathrm{~Hz}, \mathrm{PCH}_{2} \mathrm{CHCH}_{2}\right), 65.1\left(\mathrm{OCH}_{2} \mathrm{Ph}\right), 122.3(\mathrm{~d}, J$ $\left.=14.2 \mathrm{~Hz}, \mathrm{PCH}_{2} \mathrm{CHCH}_{2}\right), 125.6\left(\mathrm{~d}, J=9.3 \mathrm{~Hz}, \mathrm{PCH}_{2} \mathrm{CHCH}_{2}\right)$, $127.0(\mathrm{Ph}), 127.5(\mathrm{Ph}), 128.5(\mathrm{Ph}) ;{ }^{31} \mathrm{P} \mathrm{NMR}\left(202.45 \mathrm{MHz}, \mathrm{CDCl}_{3}\right)$ $\delta 34.5$.

1-Butylphosphinic Acid Benzyl Ester (25). Colorless oil, 15\% yield; ${ }^{1} \mathrm{H}$ NMR $\left(500.13 \mathrm{MHz}, \mathrm{CDCl}_{3}\right) \delta 0.91(\mathrm{t}, 1 \mathrm{H}, J=7.2 \mathrm{~Hz}$, $\left.\mathrm{CH}_{3}\right), 1.41$ (dist. sext., $\left.2 \mathrm{H}, J=7.4 \mathrm{~Hz}, \mathrm{P}\left(\mathrm{CH}_{2}\right)_{2} \mathrm{CH}_{2} \mathrm{CH}_{3}\right), 1.53-$ $1.62\left(\mathrm{~m}, 2 \mathrm{H}, \mathrm{PCH}_{2} \mathrm{CH}_{2} \mathrm{CH}_{2} \mathrm{CH}_{3}\right), 1.75-1.83\left(\mathrm{~m}, 2 \mathrm{H}, \mathrm{PCH}_{2}\left(\mathrm{CH}_{2}\right)_{2}-\right.$ $\left.\mathrm{CH}_{3}\right), 5.03\left(\mathrm{dd}, 1 \mathrm{H}, J_{\mathrm{H}-\mathrm{H}}=11.9 \mathrm{~Hz}, J_{\mathrm{P}-\mathrm{H}}=8.4 \mathrm{~Hz}, \mathrm{PCH} H_{\mathrm{a}} \mathrm{Ph}\right)$, $5.14\left(\mathrm{dd}, 1 \mathrm{H}, J_{\mathrm{H}-\mathrm{H}}=11.8 \mathrm{~Hz}, J_{\mathrm{P}-\mathrm{H}}=10.1 \mathrm{~Hz}, \mathrm{PCH} H_{\mathrm{b}} \mathrm{Ph}\right), 7.32-$ $7.41(\mathrm{~m}, 5 \mathrm{H}, \mathrm{Ph}), 7.65\left(\mathrm{dt}, 1 \mathrm{H}, J_{\mathrm{P}-\mathrm{H}}=530.0 \mathrm{~Hz}, J_{\mathrm{H}-\mathrm{H}}=2.0 \mathrm{~Hz}\right.$, $H \mathrm{P}) ;{ }^{13} \mathrm{C} \mathrm{NMR}\left(125.77 \mathrm{MHz}, \mathrm{CDCl}_{3}\right) \delta 13.5\left(\mathrm{PCH}_{2}\left(\mathrm{CH}_{2}\right)_{2} \mathrm{CH}_{3}\right)$, 22.7 (d, $J=2.5 \mathrm{~Hz}, \mathrm{PCH}_{2} \mathrm{CH}_{2} \mathrm{CH}_{2} \mathrm{CH}_{3}$ ), 23.5 (d, $J=16.1 \mathrm{~Hz}$, $\left.\mathrm{PCH}_{2} \mathrm{CH}_{2} \mathrm{CH}_{2} \mathrm{CH}_{3}\right), 28.5$ (d, $\left.J=92.4 \mathrm{~Hz}, \mathrm{PCH}_{2}\left(\mathrm{CH}_{2}\right)_{2} \mathrm{CH}_{3}\right), 67.5$ $\left(\mathrm{d}, J=6.8 \mathrm{~Hz}, \mathrm{OCH}_{2} \mathrm{Ph}\right), 128.1(\mathrm{Ph}), 128.6(\mathrm{Ph}), 128.7(\mathrm{Ph}), 135.8$ $(\mathrm{d}, J=5.9 \mathrm{~Hz}) ;{ }^{31} \mathrm{P}$ NMR $\left(202.45 \mathrm{MHz}, \mathrm{CDCl}_{3}\right) \delta 39.2$.

2-Pentenylphosphinic Acid Benzyl Ester (26). Colorless oil, $45 \%$ yield; ${ }^{1} \mathrm{H} \mathrm{NMR}\left(200 \mathrm{MHz}, \mathrm{CDCl}_{3}\right) \delta 0.97(\mathrm{t}, J=7.5 \mathrm{~Hz}, 3 \mathrm{H}$, $\left.\mathrm{PCH}_{2} \mathrm{CHCHCH}_{2} \mathrm{CH}_{3}\right), 2.01-2.11\left(\mathrm{~m}, 2 \mathrm{H}, \mathrm{PCH}_{2} \mathrm{CHCHCH}_{2} \mathrm{CH}_{3}\right.$ ), $2.50-2.63\left(\mathrm{~m}, 2 \mathrm{H}, \mathrm{PCH}_{2} \mathrm{CHCHCH}_{2} \mathrm{CH}_{3}\right), 5.04\left(\mathrm{dd}, J_{\mathrm{H}-\mathrm{H}}=11.9\right.$ $\left.\mathrm{Hz}, J_{\mathrm{P}-\mathrm{H}}=8.7 \mathrm{~Hz}, 1 \mathrm{H}, \mathrm{OCH}_{\mathrm{a}} \mathrm{Ph}\right), 5.14\left(\mathrm{dd}, 1 \mathrm{H}, J_{\mathrm{H}-\mathrm{H}}=11.9\right.$ $\left.\mathrm{Hz}, J_{\mathrm{P}-\mathrm{H}}=10.1 \mathrm{~Hz}, \mathrm{OCH}_{\mathrm{b}} \mathrm{Ph}\right), 5.27-5.39\left(\mathrm{~m}, 1 \mathrm{H}, \mathrm{PCH}_{2} \mathrm{CH}-\right.$ $\mathrm{CHCH}_{2} \mathrm{CH}_{3}$ ), 5.62-5.72 (m, $1 \mathrm{H}, \mathrm{PCH}_{2} \mathrm{CHCHCH}_{2} \mathrm{CH}_{3}$ ), 6.99 (ddd, $\left.J_{\mathrm{P}-\mathrm{H}}=544.5 \mathrm{~Hz}, J_{\mathrm{H}-\mathrm{H}}=2.4 \mathrm{~Hz}, J_{\mathrm{H}-\mathrm{H}}=1.8 \mathrm{~Hz}, 1 \mathrm{H}, \mathrm{HP}\right), 7.31-$ $7.40(\mathrm{~m}, 5 \mathrm{H}, \mathrm{Ph})$.

2-Hexenylphosphinic Acid Benzyl Ester (27). Colorless oil, $45 \%$ yield; ${ }^{1} \mathrm{H}$ NMR $\left(200 \mathrm{MHz}, \mathrm{CDCl}_{3}\right) \delta 0.96(\mathrm{t}, J=7.7,3 \mathrm{H}$, $\left.\mathrm{PCH}_{2} \mathrm{CHCH}\left(\mathrm{CH}_{2}\right)_{2} \mathrm{CH}_{3}\right), 1.37$ (dist. sext, $J=7.3 \mathrm{~Hz}, 2 \mathrm{H}, \mathrm{PCH}_{2}-$ $\left.\mathrm{CHCHCH}_{2} \mathrm{CH}_{2} \mathrm{CH}_{3}\right), 1.92-2.09\left(\mathrm{~m}, 2 \mathrm{H}, \mathrm{PCH}_{2} \mathrm{CHCHCH}_{2} \mathrm{CH}_{2} \mathrm{CH}_{3}\right)$, $2.51-2.58\left(\mathrm{~m}, 1 \mathrm{H}, \mathrm{PCH} \mathrm{H}_{\mathrm{a}} \mathrm{CHCH}\left(\mathrm{CH}_{2}\right)_{2} \mathrm{CH}_{3}\right), 2.60-2.67(\mathrm{~m}, 1 \mathrm{H}$, $\left.\mathrm{PCH} H_{\mathrm{b}} \mathrm{CHCH}\left(\mathrm{CH}_{2}\right)_{2} \mathrm{CH}_{3}\right), 5.03\left(\mathrm{dd}, 1 \mathrm{H}, J_{\mathrm{P}-\mathrm{H}}=8.9 \mathrm{~Hz}, J_{\mathrm{H}-\mathrm{H}}=\right.$ $\left.12.0 \mathrm{~Hz}, \mathrm{OCH} H_{\mathrm{a}} \mathrm{Ph}\right), 5.14\left(\mathrm{dd}, 1 \mathrm{H}, J_{\mathrm{P}-\mathrm{H}}=10.2 \mathrm{~Hz}, J_{\mathrm{H}-\mathrm{H}}=12.0\right.$ $\left.\mathrm{Hz}, \mathrm{OCH} H_{\mathrm{b}} \mathrm{Ph}\right), 5.21-5.41\left(\mathrm{~m}, 1 \mathrm{H}, \mathrm{PCH}_{2} \mathrm{CHCH}\left(\mathrm{CH}_{2}\right)_{2} \mathrm{CH}_{3}\right), 5.51-$ $5.75\left(\mathrm{~m}, 1 \mathrm{H}, \mathrm{PCH}_{2} \mathrm{CHCH}\left(\mathrm{CH}_{2}\right)_{2} \mathrm{CH}_{3}\right), 6.98\left(\mathrm{dt}, 1 \mathrm{H}, J_{\mathrm{P}-\mathrm{H}}=543.7\right.$ $\left.\mathrm{Hz}, J_{\mathrm{H}-\mathrm{H}}=2.1 \mathrm{~Hz}, H \mathrm{P}\right), 7.29-7.44$ (m, 5H, Ph).

2-Heptenylphosphinic Acid Benzyl Ester (28). Colorless oil, $55 \%$ yield; ${ }^{1} \mathrm{H}$ NMR $\left(200 \mathrm{MHz}, \mathrm{CDCl}_{3}\right) \delta 0.88(\mathrm{t}, J=6.9 \mathrm{~Hz}, 3 \mathrm{H}$, $\left.\mathrm{PCH}_{2} \mathrm{CHCH}\left(\mathrm{CH}_{2}\right)_{3} \mathrm{CH}_{3}\right), 1.17-1.38\left(\mathrm{~m}, 4 \mathrm{H}, \mathrm{PCH}_{2} \mathrm{CHCHCH}_{2}-\right.$ $\left.\left(\mathrm{CH}_{2}\right)_{2} \mathrm{CH}_{3}\right), 1.94-2.08\left(\mathrm{~m}, 2 \mathrm{H}, \mathrm{PCH}_{2} \mathrm{CHCHCH}_{2} \mathrm{CH}_{2} \mathrm{CH}_{2} \mathrm{CH}_{3}\right)$, $2.47-2.58\left(\mathrm{~m}, 1 \mathrm{H}, \mathrm{PCH} \mathrm{H}_{\mathrm{a}} \mathrm{CHCH}\left(\mathrm{CH}_{2}\right)_{3} \mathrm{CH}_{3}\right), 2.60-2.68(\mathrm{~m}, 1 \mathrm{H}$, $\left.\mathrm{PCH} H_{\mathrm{b}} \mathrm{CHCH}\left(\mathrm{CH}_{2}\right)_{3} \mathrm{CH}_{3}\right), 5.04\left(\mathrm{dd}, J_{\mathrm{H}-\mathrm{H}}=12.0 \mathrm{~Hz}, J_{\mathrm{P}-\mathrm{H}}=10.0\right.$ $\left.\mathrm{Hz}, 1 \mathrm{H}, \mathrm{OCH} H_{\mathrm{a}} \mathrm{Ph}\right), 5.15\left(\mathrm{dd}, J_{\mathrm{H}-\mathrm{H}}=12.0 \mathrm{~Hz}, J_{\mathrm{P}-\mathrm{H}}=10.0 \mathrm{~Hz}\right.$, $\left.1 \mathrm{H}, \mathrm{OCH} H_{\mathrm{b}} \mathrm{Ph}\right), 5.19-5.43\left(\mathrm{~m}, 1 \mathrm{H}, \mathrm{PCH}_{2} \mathrm{CHCH}\left(\mathrm{CH}_{2}\right)_{3} \mathrm{CH}_{3}\right), 5.57-$ $5.78\left(\mathrm{~m}, 1 \mathrm{H}, \mathrm{PCH}_{2} \mathrm{CHCH}\left(\mathrm{CH}_{2}\right)_{3} \mathrm{CH}_{3}\right), 6.99\left(\mathrm{dt}, J_{\mathrm{P}-\mathrm{H}}=543.5 \mathrm{~Hz}\right.$, $\left.J_{\mathrm{H}-\mathrm{H}}=2.1 \mathrm{~Hz}, 1 \mathrm{H}, H \mathrm{P}\right), 7.28-7.40(\mathrm{~m}, 5 \mathrm{H}, \mathrm{Ph})$.

General Procedure for the Preparation of $O$-Benzyl Alkyl [( $N$-Tritylamine $)$ methyl $]$ phosphinates. To a solution of methylene tritylamine $(726 \mathrm{mg}, 2.68 \mathrm{mmol})$ in anhydrous toluene $(20 \mathrm{~mL})$ cooled at $0{ }^{\circ} \mathrm{C}$ was added phosphinic acid benzyl ester $(2.30 \mathrm{mmol})$ under argon atmosphere. A $5 \% \mathrm{v} / \mathrm{v}$ solution of boron trifluoride etherate in toluene $(5 \mathrm{~mL})$ was added dropwise, and the reaction mixture was allowed to reach room temperature. The mixture was stirred for $72 \mathrm{~h}$. The solvent was evaporated, and the residue was purified by column chromatography (silica gel) employing a mixture of hexane-AcOEt (4:1) as eluant to afford the pure product.

$O$-Benzyl Methyl[( $N$-tritylamino)methyl $]$ phosphinate (29). Colorless oil, $46 \%$ yield; ${ }^{1} \mathrm{H}$ NMR (500.13 MHz, $\left.\mathrm{CD}_{3} \mathrm{OD}\right) \delta 1.63$ $\left(\mathrm{d}, J=14.0 \mathrm{~Hz}, 3 \mathrm{H}, \mathrm{CH}_{3}\right), 2.30\left(\mathrm{dd}, J_{\mathrm{H}-\mathrm{H}}=14.6, J_{\mathrm{P}-\mathrm{H}}=10.2\right.$ $\left.\mathrm{Hz}, 1 \mathrm{H}, \mathrm{PC} H_{\mathrm{a}} \mathrm{NH}\right), 2.38\left(\mathrm{dd}, J_{\mathrm{H}-\mathrm{H}}=14.6 \mathrm{~Hz}, J_{\mathrm{P}-\mathrm{H}}=11.6 \mathrm{~Hz}\right.$, $\left.1 \mathrm{H}, \mathrm{PCH}_{\mathrm{b}} \mathrm{NH}\right), 5.01\left(\mathrm{~d}, J_{\mathrm{P}-\mathrm{H}}=9.1 \mathrm{~Hz}, 2 \mathrm{H}, \mathrm{OCH}_{2} \mathrm{Ph}\right), 7.06-7.52$ $(\mathrm{m}, 20 \mathrm{H}, \mathrm{Ph}) ;{ }^{13} \mathrm{C}$ NMR $\left(125.77 \mathrm{MHz}, \mathrm{CD}_{3} \mathrm{OD}\right) \delta 12.9(\mathrm{~d}, J=$ 93.2 Hz, $\left.\mathrm{PCH}_{3}\right), 43.9\left(\mathrm{~d}, J=107.7 \mathrm{~Hz}, \mathrm{PCH}_{2} \mathrm{NH}\right), 67.6$ (d, $J=$ $\left.5.9 \mathrm{~Hz}, \mathrm{OCH}_{2} \mathrm{Ph}\right), 72.7\left(\mathrm{~d}, J=17.0 \mathrm{~Hz}, \mathrm{CPh}_{3}\right), 127.7(\mathrm{Ph}), 128.9$ $(\mathrm{Ph}), 129.0(\mathrm{Ph}), 129.6(\mathrm{Ph}), 129.7(\mathrm{Ph}), 129.8(\mathrm{Ph}), 137.9(\mathrm{~d}, J=$ $5.1 \mathrm{~Hz}, \mathrm{Ph}), 146.2(\mathrm{Ph}) ;{ }^{31} \mathrm{P}$ NMR $\left(202.45 \mathrm{MHz}, \mathrm{CD}_{3} \mathrm{OD}\right) \delta$ 56.6.

$O$-Benzyl Ethyl[ $(N$-tritylamino)methyl]phosphinate (30). Colorless oil, $52 \%$ yield; ${ }^{1} \mathrm{H}$ NMR $\left(500.13 \mathrm{MHz}, \mathrm{CDCl}_{3}\right) \delta 1.18$ (dt, $\left.J_{\mathrm{P}-\mathrm{H}}=18.1 \mathrm{~Hz}, J_{\mathrm{H}-\mathrm{H}}=7.7 \mathrm{~Hz}, 3 \mathrm{H}, \mathrm{CH}_{3}\right), 1.65-2.13(\mathrm{bs}, 1 \mathrm{H}$, $\mathrm{NH}), 1.94\left(\mathrm{dq}, 2 \mathrm{H}, J_{\mathrm{H}-\mathrm{P}}=13.9 \mathrm{~Hz}, J_{\mathrm{H}-\mathrm{H}}=7.7 \mathrm{~Hz}, \mathrm{PCH}_{2} \mathrm{CH}_{3}\right)$, $2.43\left(\mathrm{dd}, J_{\mathrm{H}-\mathrm{H}}=14.1 \mathrm{~Hz}, J_{\mathrm{H}-\mathrm{P}}=10.0 \mathrm{~Hz}, 1 \mathrm{H}, \mathrm{PCH} H_{\mathrm{a}} \mathrm{NH}\right), 2.53$ $\left(\mathrm{dd}, J_{\mathrm{H}-\mathrm{H}}=14.1 \mathrm{~Hz}, J_{\mathrm{H}-\mathrm{P}}=12.1 \mathrm{~Hz}, 1 \mathrm{H}, \mathrm{PCH} H_{\mathrm{b}} \mathrm{NH}\right), 4.98(\mathrm{dd}$, $\left.J_{\mathrm{H}-\mathrm{H}}=12.1 \mathrm{~Hz}, J_{\mathrm{P}-\mathrm{H}}=7.7 \mathrm{~Hz}, 1 \mathrm{H}, \mathrm{OCH}_{\mathrm{a}} \mathrm{Ph}\right), 5.04\left(\mathrm{dd}, J_{\mathrm{H}-\mathrm{H}}=\right.$ $\left.12.1 \mathrm{~Hz}, J_{\mathrm{P}-\mathrm{H}}=8.1 \mathrm{~Hz}, 1 \mathrm{H}, \mathrm{OCH}_{\mathrm{b}} \mathrm{Ph}\right), 7.15-7.43(\mathrm{~m}, 20 \mathrm{H}, \mathrm{Ph})$; ${ }^{13} \mathrm{C}$ NMR $\left(125.77 \mathrm{MHz}, \mathrm{CDCl}_{3}\right) \delta 5.7\left(\mathrm{~d}, J=5.1 \mathrm{~Hz}, \mathrm{PCH}_{2} \mathrm{CH}_{3}\right)$, $20.4\left(\mathrm{~d}, J=93.3 \mathrm{~Hz}, \mathrm{PCH}_{2} \mathrm{CH}_{3}\right), 41.0\left(\mathrm{~d}, J=101.7 \mathrm{~Hz}, \mathrm{PCH}_{2-}\right.$ $\mathrm{NH}), 66.0\left(\mathrm{~d}, J=5.9 \mathrm{~Hz}, \mathrm{OCH}_{2} \mathrm{Ph}\right), 71.4\left(\mathrm{~d}, J=15.3 \mathrm{~Hz}, C \mathrm{Ph}_{3}\right)$, $126.6(\mathrm{Ph}), 127.7(\mathrm{Ph}), 128.0(\mathrm{Ph}), 128.5(\mathrm{Ph}), 128.6(\mathrm{Ph}), 136.8$ $(\mathrm{d}, J=5.9 \mathrm{~Hz}, \mathrm{Ph}), 144.7(\mathrm{Ph}) ;{ }^{31} \mathrm{P} \mathrm{NMR}\left(202.45 \mathrm{MHz}, \mathrm{CDCl}_{3}\right)$ $\delta$ 57.0.

$O$-Benzyl 2-Propenyl[( $N$-tritylamino)methyl]phosphinate (31). Colorless oil, $70 \%$ yield; ${ }^{1} \mathrm{H}$ NMR $\left(500.13 \mathrm{MHz}, \mathrm{CDCl}_{3}\right) \delta 2.05$ $\left(\mathrm{dt}, 1 \mathrm{H}, J_{\mathrm{H}-\mathrm{P}}=8.0 \mathrm{~Hz}, J_{\mathrm{H}-\mathrm{H}}=3.3 \mathrm{~Hz}, \mathrm{NH}\right), 2.49\left(\mathrm{ddd}, J_{\mathrm{H}-\mathrm{H}}=\right.$ $\left.14.4 \mathrm{~Hz}, J_{\mathrm{P}-\mathrm{H}}=10.0 \mathrm{~Hz}, J_{\mathrm{H}-\mathrm{H}}=7.5,1 \mathrm{H}, \mathrm{PCH} H_{\mathrm{a}} \mathrm{NH}\right), 2.56(\mathrm{ddd}$, $\left.J_{\mathrm{H}-\mathrm{H}}=14.2 \mathrm{~Hz}, J_{\mathrm{P}-\mathrm{H}}=12.0 \mathrm{~Hz}, J_{\mathrm{H}-\mathrm{H}}=8.7,1 \mathrm{H}, \mathrm{PCH} H_{\mathrm{b}} \mathrm{NH}\right)$, $2.82\left(\mathrm{dd}, J_{\mathrm{P}-\mathrm{H}}=17.2 \mathrm{~Hz}, J_{\mathrm{H}-\mathrm{H}}=7.4,2 \mathrm{H}, \mathrm{PCH}_{2} \mathrm{CHCH}_{2}\right), 5.01$ $\left(\mathrm{dd}, J_{\mathrm{H}-\mathrm{H}}=12.0, J_{\mathrm{P}-\mathrm{H}}=8.1 \mathrm{~Hz}, 1 \mathrm{H}, \mathrm{OCH}_{\mathrm{a}} \mathrm{Ph}\right), 5.05\left(\mathrm{dd}, J_{\mathrm{H}-\mathrm{H}}=\right.$ $\left.12.0, J_{\mathrm{P}-\mathrm{H}}=8.2 \mathrm{~Hz}, 1 \mathrm{H}, \mathrm{OCH}_{\mathrm{b}} \mathrm{Ph}\right), 5.18-5.26\left(\mathrm{~m}, 3 \mathrm{H}, \mathrm{PCH}_{2}-\right.$ $\left.\mathrm{CHCH}_{2}\right), 5.83\left(\mathrm{dddt},{ }^{3} J_{\mathrm{H}-\mathrm{H} \text {,trans }}=17.2 \mathrm{~Hz},{ }^{3} J_{\mathrm{H}-\mathrm{H}, \mathrm{cis}}=9.9 \mathrm{~Hz}, J_{\mathrm{P}-\mathrm{H}}\right.$ $\left.=5.9 \mathrm{~Hz},{ }^{3} J_{\mathrm{H}-\mathrm{H}}=7.3 \mathrm{~Hz}, 1 \mathrm{H}, \mathrm{PCH}_{2} \mathrm{CHCH}_{2}\right), 7.16-7.42(\mathrm{~m}, 2 \mathrm{H}$, $\mathrm{Ph}) ;{ }^{13} \mathrm{C}$ NMR $\left(125.77 \mathrm{MHz}, \mathrm{CDCl}_{3}\right) \delta 33.6\left(\mathrm{~d}, J=88.2 \mathrm{~Hz}, \mathrm{PCH}_{2^{-}}\right.$ $\left.\mathrm{CHCH}_{2}\right), 41.2\left(\mathrm{~d}, J=104.3 \mathrm{~Hz}, \mathrm{PCH}_{2} \mathrm{NH}\right), 66.3(\mathrm{~d}, J=6.8 \mathrm{~Hz}$, $\left.\mathrm{OCH}_{2} \mathrm{Ph}\right), 71.5\left(\mathrm{~d}, J=16.1 \mathrm{~Hz}, C \mathrm{Ph}_{3}\right), 120.4(\mathrm{~d}, J=12.7 \mathrm{~Hz}$, $\left.\mathrm{PCH}_{2} \mathrm{CHCH}_{2}\right), 126.6(\mathrm{Ph}), 127.2\left(\mathrm{~d}, J=8.5 \mathrm{~Hz}, \mathrm{PCH}_{2} \mathrm{CHCH}_{2}\right)$, $127.7(\mathrm{Ph}), 128.0(\mathrm{Ph}), 128.3(\mathrm{Ph}), 128.5(\mathrm{Ph}), 128.6(\mathrm{Ph}), 136.5$ $(\mathrm{d}, J=5.1 \mathrm{~Hz}, \mathrm{Ph}) ;{ }^{31} \mathrm{P}$ NMR $\left(202.45 \mathrm{MHz}, \mathrm{CDCl}_{3}\right) \delta 51.5$.

O-Benzyl 1-Butyl[( $N$-tritylamino)methyl]phosphinate (32). Colorless oil, $51 \%$ yield; ${ }^{1} \mathrm{H}$ NMR $\left(500.13 \mathrm{MHz}, \mathrm{CDClD}_{3}\right) \delta 0.92$ (t, $J=7.3 \mathrm{~Hz}, 3 \mathrm{H}, \mathrm{CH}_{3}$ ), $1.37-1.48$ (dist. sext, $J=7.3 \mathrm{~Hz}, 2 \mathrm{H}$, $\left.\mathrm{P}\left(\mathrm{CH}_{2}\right)_{2} \mathrm{CH}_{2} \mathrm{CH}_{3}\right), 1.51-1.64$ (m, 2H, $\left.\mathrm{PCH}_{2} \mathrm{CH}_{2} \mathrm{CH}_{2} \mathrm{CH}_{3}\right), 1.85-$ $2.09\left(\mathrm{~m}, 3 \mathrm{H}, \mathrm{PCH}_{2}\left(\mathrm{CH}_{2}\right)_{2} \mathrm{CH}_{3}\right), 2.42\left(\mathrm{dd}, J_{\mathrm{H}-\mathrm{H}}=14.2 \mathrm{~Hz}, J_{\mathrm{H}-\mathrm{P}}=\right.$ $\left.10.0 \mathrm{~Hz}, 1 \mathrm{H}, \mathrm{PCH} H_{\mathrm{a}} \mathrm{NH}\right), 2.53\left(\mathrm{dd}, J_{\mathrm{H}-\mathrm{H}}=14.2 \mathrm{~Hz}, J_{\mathrm{H}-\mathrm{P}}=12.1\right.$ $\left.\mathrm{Hz} 1 \mathrm{H}, \mathrm{PCH} H_{\mathrm{b}} \mathrm{NH}\right), 4.98\left(\mathrm{dd}, J_{\mathrm{H}-\mathrm{H}}=12.0 \mathrm{~Hz}, J_{\mathrm{H}-\mathrm{P}}=7.9 \mathrm{~Hz}\right.$, $\left.1 \mathrm{H}, \mathrm{OCH}_{\mathrm{a}} \mathrm{Ph}\right), 5.04\left(\mathrm{dd}, J_{\mathrm{H}-\mathrm{H}}=12.0 \mathrm{~Hz}, J_{\mathrm{H}-\mathrm{P}}=8.1 \mathrm{~Hz}, 1 \mathrm{H}, \mathrm{OCH}_{\mathrm{b}^{-}}\right.$ $\mathrm{Ph}), 7.12-7.47(\mathrm{~m}, 20 \mathrm{H}, \mathrm{Ph}) ;{ }^{13} \mathrm{C} \mathrm{NMR}\left(125.77 \mathrm{MHz}, \mathrm{CDCl}_{3}\right) \delta$

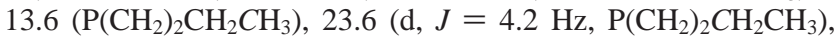
$23.9\left(\mathrm{~d}, J=16.1 \mathrm{~Hz}, \mathrm{PCH}_{2} \mathrm{CH}_{2} \mathrm{CH}_{2} \mathrm{CH}_{3}\right), 27.1(\mathrm{~d}, J=91.6 \mathrm{~Hz}$, $\left.\mathrm{PCH}_{2}\left(\mathrm{CH}_{2}\right)_{2} \mathrm{CH}_{3}\right), 41.4$ (d, $\left.J=100.1 \mathrm{~Hz}, \mathrm{PCH}_{2} \mathrm{NH}\right), 65.9$ (d, $J=$ $\left.5.9 \mathrm{~Hz}, \mathrm{OCH}_{2} \mathrm{Ph}\right), 71.4\left(\mathrm{~d}, J=15.3 \mathrm{~Hz}, C \mathrm{Ph}_{3}\right), 126.7(\mathrm{Ph}), 127.8$ $(\mathrm{Ph}), 128.1(\mathrm{Ph}), 128.4(\mathrm{Ph}), 128.6(\mathrm{Ph}) 128.7(\mathrm{Ph}), 136.8(\mathrm{~d}, J=$ 5.1, Ph), 144.8 (Ph); ${ }^{31} \mathrm{P}$ NMR (202.45 MHz, $\left.\mathrm{CDCl}_{3}\right) \delta 56.1$.

$O$-Benzyl 2-Pentenyl[( $N$-tritylamino)methyl $]$ phosphinate (33). Colorless oil, $47 \%$ yield; ${ }^{1} \mathrm{H}$ NMR $\left(500.13 \mathrm{MHz}, \mathrm{CDClD}_{3}\right) \delta 0.94$ $\left(\mathrm{t}, J=7.4 \mathrm{~Hz}, 3 \mathrm{H}, \mathrm{PCH}_{2} \mathrm{CHCHCH}_{2} \mathrm{CH}_{3}\right), 1.96-2.08\left(\mathrm{~m}, 3 \mathrm{H}, \mathrm{PCH}_{2}-\right.$ $\mathrm{CHCHCH}_{2} \mathrm{CH}_{3}, \mathrm{NH}$ ), 2.46 (dist. t, $J=11.9 \mathrm{~Hz}, 1 \mathrm{H}, \mathrm{PCH}_{\mathrm{a}} \mathrm{NH}$ ), 2.54 (dist. t, $\left.J=13.6 \mathrm{~Hz}, 1 \mathrm{H}, \mathrm{PC} H_{\mathrm{b}} \mathrm{NH}\right), 2.74\left(\mathrm{dd}, J_{\mathrm{P}-\mathrm{H}}=16.8\right.$ $\left.\mathrm{Hz}, J_{\mathrm{H}-\mathrm{H}}=7.3,2 \mathrm{H}, \mathrm{PCH}_{2} \mathrm{CHCHCH}_{2} \mathrm{CH}_{3}\right), 5.00\left(\mathrm{dd}, J_{\mathrm{H}-\mathrm{H}}=12.0\right.$ $\left.\mathrm{Hz}, J_{\mathrm{H}-\mathrm{P}}=7.9 \mathrm{~Hz}, 1 \mathrm{H}, \mathrm{OCH}_{\mathrm{a}} \mathrm{Ph}\right), 5.06\left(\mathrm{dd}, J_{\mathrm{H}-\mathrm{H}}=12.0 \mathrm{~Hz}\right.$, $\left.J_{\mathrm{H}-\mathrm{P}}=7.9 \mathrm{~Hz}, 1 \mathrm{H}, \mathrm{OCH}_{\mathrm{b}} \mathrm{Ph}\right), 5.36-5.46\left(\mathrm{~m}, 1 \mathrm{H}, \mathrm{PCH}_{2} \mathrm{CHCHCH}_{2^{-}}\right.$ $\left.\mathrm{CH}_{3}\right), 5.62-5.71\left(\mathrm{~m}, 1 \mathrm{H}, \mathrm{PCH}_{2} \mathrm{CHCHCH}_{2} \mathrm{CH}_{3}\right), 7.14-7.51(\mathrm{~m}$, $20 \mathrm{H}, \mathrm{Ph}) ;{ }^{13} \mathrm{C}$ NMR $\left(125.77 \mathrm{MHz}, \mathrm{CDCl}_{3}\right) \delta 13.7\left(\mathrm{PCH}_{2-}\right.$ $\mathrm{CHCHCH}_{2} \mathrm{CH}_{3}$ ), 25.7 (d, $J=2.5 \mathrm{~Hz}, \mathrm{PCH}_{2} \mathrm{CHCHCH}_{2} \mathrm{CH}_{3}$ ), 32.3 $\left(\mathrm{d}, J=89.0 \mathrm{~Hz}, \mathrm{PCH}_{2} \mathrm{CHCHCH}_{2} \mathrm{CH}_{3}\right), 41.0(\mathrm{~d}, J=103.4 \mathrm{~Hz}$, $\left.\mathrm{PCH}_{2} \mathrm{NH}\right), 66.1\left(\mathrm{~d}, J=6.8 \mathrm{~Hz}, \mathrm{OCH}_{2} \mathrm{Ph}\right), 71.4(\mathrm{~d}, J=16.1 \mathrm{~Hz}$, $\left.C \mathrm{Ph}_{3}\right), 117.2\left(\mathrm{~d}, J=9.3 \mathrm{~Hz}, \mathrm{PCH}_{2} \mathrm{CHCHCH}_{2} \mathrm{CH}_{3}\right), 126.6(\mathrm{Ph})$, $127.7(\mathrm{Ph}), 128.0(\mathrm{Ph}), 128.3(\mathrm{Ph}), 128.5(\mathrm{Ph}), 128.6(\mathrm{Ph}), 136.6$ $(\mathrm{d}, J=5.9 \mathrm{~Hz}, \mathrm{Ph}), 138.2\left(\mathrm{~d}, J=11.9 \mathrm{~Hz}, \mathrm{PCH}_{2} \mathrm{CHCHCH}_{2} \mathrm{CH}_{3}\right)$, $144.7(\mathrm{Ph}) ;{ }^{31} \mathrm{P}$ NMR $\left(202.45 \mathrm{MHz}, \mathrm{CDCl}_{3}\right) \delta 52.5$.

$\boldsymbol{O}$-Benzyl 2-Hexenyl[( $\boldsymbol{N}$-tritylamino)methyl $]$ phosphinate (34). Colorless oil, $74 \%$ yield; ${ }^{1} \mathrm{H}$ NMR $\left(500.13 \mathrm{MHz}, \mathrm{CDClD}_{3}\right) \delta 0.85$ (t, $J=7.4 \mathrm{~Hz}, \mathrm{PCH}_{2} \mathrm{CHCH}\left(\mathrm{CH}_{2}\right)_{2} \mathrm{CH}_{3}$ ), 1.32 (dist. sext, $J=7.3$ $\left.\mathrm{Hz}, 2 \mathrm{H}, \mathrm{PCH}_{2} \mathrm{CHCHCH}_{2} \mathrm{CH}_{2} \mathrm{CH}_{3}\right), 1.93-2.00\left(\mathrm{~m}, 2 \mathrm{H}, \mathrm{PCH}_{2-}\right.$ $\left.\mathrm{CHCHCH}_{2} \mathrm{CH}_{2} \mathrm{CH}_{3}\right), 2.00-2.08$ (bs, $\left.1 \mathrm{H}, \mathrm{NH}\right), 2.41-2.60(\mathrm{~m}, 2 \mathrm{H}$, $\left.\mathrm{PCH}_{2} \mathrm{NH}\right), 2.74\left(\mathrm{dd}, \mathrm{J}_{\mathrm{H}-\mathrm{H}}=7.3, J_{\mathrm{P}-\mathrm{H}}=17.1 \mathrm{~Hz}, 2 \mathrm{H}, \mathrm{PCH}_{2} \mathrm{CHCH}-\right.$ 
$\left.\left(\mathrm{CH}_{2}\right)_{2} \mathrm{CH}_{3}\right), 5.01\left(\mathrm{dd}, J_{\mathrm{H}-\mathrm{H}}=12.1 \mathrm{~Hz}, J_{\mathrm{P}-\mathrm{P}}=7.8 \mathrm{~Hz}, 1 \mathrm{H}, \mathrm{OCH} H_{\mathrm{a}-}\right.$ $\mathrm{Ph}), 5.06\left(\mathrm{dd}, J_{\mathrm{H}-\mathrm{H}}=12.2 \mathrm{~Hz}, J_{\mathrm{P}-\mathrm{P}}=7.8 \mathrm{~Hz}, 1 \mathrm{H}, \mathrm{OCH} H_{\mathrm{b}} \mathrm{Ph}\right)$, 5.37-5.46 (m, $\left.1 \mathrm{H}, \mathrm{PCH}_{2} \mathrm{CHCH}\left(\mathrm{CH}_{2}\right)_{2} \mathrm{CH}_{3}\right), 5.57-5.65(\mathrm{~m}, 1 \mathrm{H}$, $\left.\mathrm{PCH}_{2} \mathrm{CHCH}\left(\mathrm{CH}_{2}\right)_{2} \mathrm{CH}_{3}\right), 7.14-7.44(\mathrm{~m}, 20 \mathrm{H}) ;{ }^{13} \mathrm{C}$ NMR $(125.77$ $\left.\mathrm{MHz}, \mathrm{CDCl}_{3}\right) \delta 13.5\left(\mathrm{PCH}_{2} \mathrm{CHCH}\left(\mathrm{CH}_{2}\right)_{2} \mathrm{CH}_{3}\right), 22.1(\mathrm{~d}, J=3.4$ $\left.\mathrm{Hz}, \mathrm{PCH}_{2} \mathrm{CHCH}\left(\mathrm{CH}_{2}\right) \mathrm{CH}_{2} \mathrm{CH}_{3}\right), 32.2\left(\mathrm{~d}, J=89.0 \mathrm{~Hz}, \mathrm{PCH}_{2-}\right.$ $\left.\mathrm{CHCH}\left(\mathrm{CH}_{2}\right)_{2} \mathrm{CH}_{3}\right), 35.9$ (d, $J=1.7 \mathrm{~Hz}, \mathrm{PCH}_{2} \mathrm{CHCHCH}_{2} \mathrm{CH}_{2} \mathrm{CH}_{3}$ ), $40.9\left(\mathrm{~d}, J=103.4 \mathrm{~Hz}, \mathrm{PCH}_{2} \mathrm{NH}\right), 66.0\left(\mathrm{~d}, J=5.9 \mathrm{~Hz}, \mathrm{OCH}_{2} \mathrm{Ph}\right)$, $71.3\left(\mathrm{~d}, J=16.1 \mathrm{~Hz}, C \mathrm{Ph}_{3}\right), 118.1\left(\mathrm{~d}, J=9.3 \mathrm{~Hz}, \mathrm{PCH}_{2} C \mathrm{HCH}-\right.$ $\left.\left(\mathrm{CH}_{2}\right)_{2} \mathrm{CH}_{3}\right), 126.4(\mathrm{Ph}), 127.6(\mathrm{Ph}), 127.8(\mathrm{Ph}), 128.1(\mathrm{Ph}), 128.38$ $(\mathrm{Ph}), 128.43(\mathrm{Ph}), 136.4\left(\mathrm{~d}, J=12.7 \mathrm{~Hz}, \mathrm{PCH}_{2} \mathrm{CHCH}\left(\mathrm{CH}_{2}\right)_{2} \mathrm{CH}_{3}\right)$, 136.5 (d, $J=5.1 \mathrm{~Hz}, \mathrm{Ph}), 144.5(\mathrm{Ph}) ;{ }^{31} \mathrm{P} \mathrm{NMR}(202.45 \mathrm{MHz}$, $\left.\mathrm{CDCl}_{3}\right) \delta 52.4$.

$O$-Benzyl 2-Heptenyl[( $N$-tritylamino)methyl $]$ phosphinate (35). Colorless oil, $74 \%$ yield; ${ }^{1} \mathrm{H}$ NMR $\left(500.13 \mathrm{MHz}, \mathrm{CDClD}_{3}\right) \delta 0.86$ $\left(\mathrm{t}, J=7.1 \mathrm{~Hz}, \mathrm{PCH}_{2} \mathrm{CHCH}\left(\mathrm{CH}_{2}\right)_{3} \mathrm{CH}_{3}\right), 1.21-1.34\left(\mathrm{~m}, 4 \mathrm{H}, \mathrm{PCH}_{2}\right.$ $\left.\mathrm{CHCHCH}_{2}\left(\mathrm{CH}_{2}\right)_{2} \mathrm{CH}_{3}\right), 1.95-2.05\left(\mathrm{~m}, 3 \mathrm{H}, \mathrm{PCH}_{2} \mathrm{CHCHCH}_{2}\left(\mathrm{CH}_{2}\right)_{2}-\right.$ $\left.\mathrm{CH}_{3}, \mathrm{NH}\right), 2.45\left(\mathrm{dd}, J_{\mathrm{H}-\mathrm{H}}=13.4 \mathrm{~Hz}, J_{\mathrm{H}-\mathrm{P}}=10.5 \mathrm{~Hz}, 1 \mathrm{H}\right.$, $\mathrm{PCH}_{\mathrm{a}} \mathrm{NH}$ ), 2.54 (dist. $\left.\mathrm{t}, J_{\mathrm{H}-\mathrm{H}}=13.1 \mathrm{~Hz}, 1 \mathrm{H}, \mathrm{PCH}_{\mathrm{b}} \mathrm{NH}\right), 2.73(\mathrm{dd}$, $\left.J_{\mathrm{P}-\mathrm{H}}=16.9 \mathrm{~Hz}, J_{\mathrm{H}-\mathrm{H}}=7.3,2 \mathrm{H}, \mathrm{PCH}_{2} \mathrm{CHCH}\left(\mathrm{CH}_{2}\right)_{3} \mathrm{CH}_{3}\right), 5.01$ $\left(\mathrm{dd}, J_{\mathrm{H}-\mathrm{H}}=12.1 \mathrm{~Hz}, J_{\mathrm{P}-\mathrm{P}}=7.7 \mathrm{~Hz}, 1 \mathrm{H}, \mathrm{OCH}_{\mathrm{a}} \mathrm{Ph}\right), 5.06\left(\mathrm{dd}, J_{\mathrm{H}-\mathrm{H}}\right.$ $\left.=12.1 \mathrm{~Hz}, J_{\mathrm{P}-\mathrm{P}}=7.7 \mathrm{~Hz}, 1 \mathrm{H}, \mathrm{OCH}_{\mathrm{b}} \mathrm{Ph}\right), 5.35-5.45(\mathrm{~m}, 1 \mathrm{H}$, $\left.\mathrm{PCH}_{2} \mathrm{CHCH}\left(\mathrm{CH}_{2}\right)_{3} \mathrm{CH}_{3}\right), 5.56-5.65\left(\mathrm{~m}, 1 \mathrm{H}, \mathrm{PCH}_{2} \mathrm{CHCH}\left(\mathrm{CH}_{2}\right)_{3}\right.$ $\left.\mathrm{CH}_{3}\right), 7.16-7.43(\mathrm{~m}, 20 \mathrm{H}) ;{ }^{13} \mathrm{C} \mathrm{NMR}\left(125.77 \mathrm{MHz}, \mathrm{CDCl}_{3}\right) \delta 13.8$ $\left(\mathrm{PCH}_{2} \mathrm{CHCH}\left(\mathrm{CH}_{2}\right)_{2} \mathrm{CH}_{2} \mathrm{CH}_{3}\right), 22.1\left(\mathrm{PCH}_{2} \mathrm{CHCH}\left(\mathrm{CH}_{2}\right)_{2} \mathrm{CH}_{2} \mathrm{CH}_{3}\right)$, $31.1\left(\mathrm{~d}, J=2.5 \mathrm{~Hz}, \mathrm{PCH}_{2} \mathrm{CHCHCH}_{2} \mathrm{CH}_{2} \mathrm{CH}_{2} \mathrm{CH}_{3}\right), 32.26(\mathrm{~d}, J=$ $\left.88.2 \mathrm{~Hz}, \mathrm{PCH}_{2} \mathrm{CHCH}\left(\mathrm{CH}_{2}\right)_{3} \mathrm{CH}_{3}\right), 32.27$ (d, $\mathrm{J}=1.7 \mathrm{~Hz}, \mathrm{PCH}_{2}$ $\mathrm{CHCHCH}_{2} \mathrm{CH}_{2} \mathrm{CH}_{2} \mathrm{CH}_{3}$ ), 41.0 (d, $J=103.4 \mathrm{~Hz}, \mathrm{PCH}_{2} \mathrm{NH}$ ), 66.1 $\left(\mathrm{d}, J=6.8 \mathrm{~Hz}, \mathrm{OCH}_{2} \mathrm{Ph}\right), 71.4\left(\mathrm{~d}, J=15.3 \mathrm{~Hz}, C \mathrm{Ph}_{3}\right), 118.0$ (d, $\left.J=9.3 \mathrm{~Hz}, \mathrm{PCH}_{2} \mathrm{CHCH}\left(\mathrm{CH}_{2}\right)_{2} \mathrm{CH}_{2} \mathrm{CH}_{3}\right), 126.5(\mathrm{Ph}), 127.6(\mathrm{Ph})$, $127.9(\mathrm{Ph}), 128.2(\mathrm{Ph}), 128.47(\mathrm{Ph}), 128.51(\mathrm{Ph}), 136.6(\mathrm{~d}, J=$ $5.9 \mathrm{~Hz}, \mathrm{Ph}), 136.8$ (d, $\left.J=11.9 \mathrm{~Hz}, \mathrm{PCH}_{2} \mathrm{CHCH}\left(\mathrm{CH}_{2}\right)_{2} \mathrm{CH}_{2} \mathrm{CH}_{3}\right)$, $144.6(\mathrm{Ph}) ;{ }^{31} \mathrm{P}$ NMR $\left(202.45 \mathrm{MHz}, \mathrm{CDCl}_{3}\right) \delta 52.5$.

General Procedure for Trityl Cleavage. To a solution of $O$-benzylmethyl[ $(N$-tritylamine $)$ alkyl $]$ phosphinate $(0.68 \mathrm{mmol})$ in methylene chloride $(5 \mathrm{~mL})$ was added dropwise a $5 \% \mathrm{v} / \mathrm{v}$ solution of trifluoroacetic acid in methylene chloride $(5 \mathrm{~mL})$ at room temperature. The reaction was stirred for $30 \mathrm{~min}$. The solvent was evaporated, and the residue was redissolved in methanol $(10 \mathrm{~mL})$. Then, strong cationic resin Amberlite CG-400 (1.00 g) was added, and the mixture was stirred for $1 \mathrm{~h}$. The mixture was filtered off and the filtrate washed with methanol $(20 \mathrm{~mL})$. The solvent was evaporated, and the residue was purified by column chromatography (silica gel) eluting with AcOEt $\rightarrow$ AcOEt - methanol (4:1) to yield the corresponding free amine as an oil that had to be rapidly used in next step to prevent $N$-oxide formation.

$O$-Benzyl Methyl(aminomethylmethyl)phosphinate (36). Pale yellow oil. 64\% yield; ${ }^{1} \mathrm{H}$ NMR $\left(500.13 \mathrm{MHz}, \mathrm{CD}_{3} \mathrm{OD}\right) \delta 1.68(\mathrm{~d}$, $\left.J=14.8 \mathrm{~Hz}, 3 \mathrm{H}, \mathrm{PCH}_{3}\right), 3.36\left(\mathrm{~d}, J=9.8 \mathrm{~Hz}, 2 \mathrm{H}, \mathrm{NH}_{2} \mathrm{CH}_{2} \mathrm{P}\right)$, $5.12\left(\mathrm{dd}, J_{\mathrm{H}-\mathrm{H}}=11.7 \mathrm{~Hz}, J_{\mathrm{H}-\mathrm{P}}=9.5 \mathrm{~Hz}, 1 \mathrm{H}, \mathrm{OCH}_{\mathrm{a}} \mathrm{Ph}\right), 5.17$ $\left(\mathrm{dd}, J_{\mathrm{H}-\mathrm{H}}=11.7 \mathrm{~Hz}, J_{\mathrm{H}-\mathrm{P}}=8.8 \mathrm{~Hz}, 1 \mathrm{H}, \mathrm{OCH} H_{\mathrm{b}} \mathrm{Ph}\right), 7.28-7.49$ (m, 5H, Ph). ${ }^{13} \mathrm{C}$ NMR $\left(125.77 \mathrm{MHz}, \mathrm{CD}_{3} \mathrm{OD}\right) \delta 13.8(\mathrm{~d}, J=96.6$, $\left.C_{3}\right), 37.7\left(\mathrm{~d}, J=97.5 \mathrm{~Hz}, \mathrm{NHCH}_{2} \mathrm{P}\right), 68.4\left(\mathrm{~d}, J=6.8 \mathrm{~Hz}, \mathrm{OCH}_{2}-\right.$ $\mathrm{Ph}), 129.4(\mathrm{Ph}), 129.8(\mathrm{Ph}), 129.9(\mathrm{Ph}), 137.2(\mathrm{~d}, J=5.9 \mathrm{~Hz}, \mathrm{Ph})$. ${ }^{31} \mathrm{P}$ NMR (202.45 MHz, $\left.\mathrm{CD}_{3} \mathrm{OD}\right) \delta$ 47.6.

$O$-Benzyl Ethyl(aminomethyl)phosphinate (37). Pale yellow oil; ${ }^{1} \mathrm{H}$ NMR $\left(500.13 \mathrm{MHz}, \mathrm{CD}_{3} \mathrm{OD}\right) \delta 1.13\left(\mathrm{dt}, J_{\mathrm{H}-\mathrm{P}}=19.1 \mathrm{~Hz}\right.$, $\left.J_{\mathrm{H}-\mathrm{H}}=7.7 \mathrm{~Hz}, 3 \mathrm{H}, \mathrm{PCH}_{2} \mathrm{CH}_{3}\right), 1.94\left(\mathrm{dq}, J_{\mathrm{H}-\mathrm{P}}=15.2 \mathrm{~Hz}, J_{\mathrm{H}-\mathrm{H}}=\right.$ $\left.7.7 \mathrm{~Hz}, 2 \mathrm{H}, \mathrm{PCH}_{2} \mathrm{CH}_{3}\right), 3.27\left(\mathrm{~d}, J=8.8 \mathrm{~Hz}, 2 \mathrm{H}, \mathrm{NHCH}_{2} \mathrm{P}\right), 5.03$ $\left(\mathrm{dd}, J_{\mathrm{H}-\mathrm{H}}=11.8 \mathrm{~Hz}, J_{\mathrm{H}-\mathrm{P}}=8.5 \mathrm{~Hz}, 1 \mathrm{H}, \mathrm{OCH}_{\mathrm{a}} \mathrm{Ph}\right), 5.15(\mathrm{dd}$, $\left.J_{\mathrm{H}-\mathrm{H}}=11.8 \mathrm{~Hz}, J_{\mathrm{H}-\mathrm{P}}=10.1 \mathrm{~Hz}, 1 \mathrm{H}, \mathrm{OCH}_{\mathrm{b}} \mathrm{Ph}\right), 7.36-7.50(\mathrm{~m}$, $5 \mathrm{H}, \mathrm{Ph})$.

$O$-Benzyl 2-Propen(aminomethylmethyl)phosphinate (38). Pale yellow oil, $89 \%$ yield; ${ }^{1} \mathrm{H}$ NMR $\left(200 \mathrm{MHz}, \mathrm{CDCl}_{3}\right) \delta 2.67$ $\left(\mathrm{dd}, J_{\mathrm{H}-\mathrm{P}}=17.9 \mathrm{~Hz}, J_{\mathrm{H}-\mathrm{H}}=7.4 \mathrm{~Hz}, 3 \mathrm{H}, \mathrm{PCH}_{2} \mathrm{NH}_{2}\right), 2.96-3.14$ (m, $2 \mathrm{H}, \mathrm{PCH}_{2} \mathrm{CHCH}_{2}$ ), 3.14-3.44 (broad s, $\left.2 \mathrm{H}, \mathrm{NH}_{2}\right), 5.05$ (d, $J$ $\left.=8.5 \mathrm{~Hz}, 2 \mathrm{H}, \mathrm{OCH}_{2} \mathrm{Ph}\right), 5.09-5.25\left(\mathrm{~m}, 2 \mathrm{H}, \mathrm{PCH}_{2} \mathrm{CHCH}_{2}\right), 5.54-$ $5.87\left(\mathrm{~m}, 1 \mathrm{H}, \mathrm{PCH}_{2} \mathrm{CHCH}_{2}\right), 7.26-7.37(\mathrm{~m}, 5 \mathrm{H}, \mathrm{Ph})$.

O-Benzyl 1-Butyl(aminomethylmethyl)phosphinate (39). Pale yellow oil. 87\% yield; ${ }^{1} \mathrm{H}$ NMR $\left(500.13 \mathrm{MHz}, \mathrm{CDCl}_{3}\right) \delta 0.81(\mathrm{t}$, $\left.J=7.3 \mathrm{~Hz}, 3 \mathrm{H}, \mathrm{PCH}_{2}\left(\mathrm{CH}_{2}\right)_{2} \mathrm{CH}_{3}\right), 1.29$ (dist. sept., $J=7.1 \mathrm{~Hz}$,
2H, $\left.\mathrm{P}\left(\mathrm{CH}_{2}\right)_{2} \mathrm{CH}_{2} \mathrm{CH}_{3}\right)$ 1.36-1.50 (m, $\left.2 \mathrm{H}, \mathrm{PCH}_{2} \mathrm{CH}_{2} \mathrm{CH}_{2} \mathrm{CH}_{3}\right), 1.85$ $\left(\mathrm{dt}, J_{\mathrm{H}-\mathrm{P}}=15.4 \mathrm{~Hz}, J_{\mathrm{H}-\mathrm{H}}=8.2 \mathrm{~Hz}, 2 \mathrm{H}, \mathrm{PCH}_{2}\left(\mathrm{CH}_{2}\right)_{2} \mathrm{CH}_{3}\right), 3.21$ $\left(\mathrm{d}, J=8.0 \mathrm{~Hz}, 2 \mathrm{H}, \mathrm{NH}_{2}\right), 5.01\left(\mathrm{dd}, J_{\mathrm{H}-\mathrm{H}}=11.8 \mathrm{~Hz}, J_{\mathrm{H}-\mathrm{P}}=8.7\right.$ $\left.\mathrm{Hz}, 1 \mathrm{H}, \mathrm{OC} H_{\mathrm{a}} \mathrm{Ph}\right), 5.09\left(\mathrm{dd}, J_{\mathrm{H}-\mathrm{H}}=11.8 \mathrm{~Hz}, J_{\mathrm{H}-\mathrm{P}}=8.4 \mathrm{~Hz}, 1 \mathrm{H}\right.$, $\left.\mathrm{OCH}_{\mathrm{b}} \mathrm{Ph}\right) ;{ }^{13} \mathrm{C}$ NMR $\left(125.77 \mathrm{MHz}, \mathrm{CDCl}_{3}\right) \delta 13.2\left(\mathrm{PCH}_{2^{-}}\right.$ $\left.\left(\mathrm{CH}_{2}\right)_{2} \mathrm{CH}_{3}\right), 23.1$ (d, $\left.J=5.1 \mathrm{~Hz}, \mathrm{PCH}_{2} \mathrm{CH}_{2} \mathrm{CH}_{2} \mathrm{CH}_{3}\right), 23.5$ (d, $J=$ $\left.17.0 \mathrm{~Hz}, \mathrm{P}\left(\mathrm{CH}_{2}\right)_{2} \mathrm{CH}_{2} \mathrm{CH}_{3}\right), 27.6$ (d, $\left.J=95.8, \mathrm{PCH}_{2}\left(\mathrm{CH}_{2}\right)_{2} \mathrm{CH}_{3}\right)$, $35.7\left(\mathrm{~d}, J=91.6 \mathrm{~Hz}, \mathrm{NHCH}_{2} \mathrm{P}\right), 67.0\left(\mathrm{~d}, J=6.8 \mathrm{~Hz}, \mathrm{OCH}_{2} \mathrm{Ph}\right)$, $127.9(\mathrm{Ph}), 128.7(\mathrm{Ph}), 135.7$ (d, $J=5.9 \mathrm{~Hz}, \mathrm{Ph}) ;{ }^{31} \mathrm{P}$ NMR $(202.45$ $\left.\mathrm{MHz}, \mathrm{CDCl}_{3}\right) \delta 50.9$.

$O$-Benzyl 2-Penten(aminomethylmethyl)phosphinate (40). Pale yellow oil. $76 \%$ yield; ${ }^{1} \mathrm{H}$ NMR $\left(500.13 \mathrm{MHz}, \mathrm{CDCl}_{3}-\mathrm{CD}_{3} \mathrm{OD}\right.$, $4: 1) \delta 0.95\left(\mathrm{t}, J=7.5 \mathrm{~Hz}, 3 \mathrm{H}, \mathrm{PCH}_{2} \mathrm{CHCHCH}_{2} \mathrm{CH}_{3}\right.$ ), 2.02 (dist. sext, $\left.J=6.8 \mathrm{~Hz}, 2 \mathrm{H}, \mathrm{PCH}_{2} \mathrm{CHCHCH}_{2} \mathrm{CH}_{3}\right), 2.60-2.75(\mathrm{~m}, 2 \mathrm{H}$, $\mathrm{PCH}_{2} \mathrm{NH}_{2}$ ), $3.04-3.25\left(\mathrm{~m}, 2 \mathrm{H}, \mathrm{PCH}_{2} \mathrm{CHCHCH}_{2} \mathrm{CH}_{3}\right.$ ), 5.07 (dd, $\left.J_{\mathrm{H}-\mathrm{H}}=11.8 \mathrm{~Hz}, J_{\mathrm{H}-\mathrm{P}}=9.0 \mathrm{~Hz}, 2 \mathrm{H}, \mathrm{OCH}_{\mathrm{a}} \mathrm{Ph}\right), 5.11\left(\mathrm{dd}, J_{\mathrm{H}-\mathrm{H}}=\right.$ $\left.11.8 \mathrm{~Hz}, J_{\mathrm{H}-\mathrm{P}}=8.3 \mathrm{~Hz}, 2 \mathrm{H}, \mathrm{OCH}_{\mathrm{b}} \mathrm{Ph}\right), 5.21-5.30(\mathrm{~m}, 2 \mathrm{H}$, $\left.\mathrm{PCH}_{2} \mathrm{CHCHCH} \mathrm{CH}_{3}\right), 5.62-5.72\left(\mathrm{~m}, 2 \mathrm{H}, \mathrm{PCH}_{2} \mathrm{CHCHCH}_{2} \mathrm{CH}_{3}\right)$, 7.31-7.44 (m, 5H, Ph).

$O$-Benzyl 2-Hexen(aminomethylmethyl)phosphinate (41). Pale yellow oil. $94 \%$ yield; ${ }^{1} \mathrm{H}$ NMR $\left(500.13 \mathrm{MHz}, \mathrm{CDCl}_{3}-\mathrm{CD}_{3} \mathrm{OD}\right.$, 4:1) $\delta 0.89\left(\mathrm{t}, J=7.4 \mathrm{~Hz}, 3 \mathrm{H}, \mathrm{CH}_{3}\right.$ ), 1.37 (dist. sext, $J=7.3 \mathrm{~Hz}$, $\left.2 \mathrm{H}, \mathrm{PCH}_{2} \mathrm{CHCHCH}_{2} \mathrm{CH}_{2} \mathrm{CH}_{3}\right), 1.95-2.05\left(\mathrm{~m}, 2 \mathrm{H}, \mathrm{PCH}_{2} \mathrm{CHCHCH}_{2}-\right.$ $\left.\mathrm{CH}_{2} \mathrm{CH}_{3}\right), 2.61-2.69\left(\mathrm{~m}, 2 \mathrm{H}, \mathrm{PCH}_{2} \mathrm{NH}\right), 2.92-3.06\left(\mathrm{~m}, 2 \mathrm{H}, \mathrm{PCH}_{2-}\right.$ $\left.\mathrm{CHCH}\left(\mathrm{CH}_{2}\right)_{2} \mathrm{CH}_{3}\right), 5.08\left(\mathrm{~d}, J=8.4 \mathrm{~Hz}, 2 \mathrm{H}, \mathrm{OCH}_{2} \mathrm{Ph}\right), 5.28-5.42$ $\left(\mathrm{m}, 2 \mathrm{H}, \mathrm{PCH}_{2} \mathrm{CHCH}\left(\mathrm{CH}_{2}\right)_{2} \mathrm{CH}_{3}\right), 5.57-5.71\left(\mathrm{~m}, 2 \mathrm{H}, \mathrm{PCH}_{2-}\right.$ $\left.\mathrm{CHCH}\left(\mathrm{CH}_{2}\right)_{2} \mathrm{CH}_{3}\right), 7.31-7.44(\mathrm{~m}, 5 \mathrm{H}, \mathrm{Ph})$.

$O$-Benzyl 2-Hepten(aminomethylmethyl)phosphinate (42). Pale yellow oil. $85 \%$ yield.

${ }^{1} \mathrm{H}$ NMR $\left(500.13 \mathrm{MHz}, \mathrm{CDCl}_{3}-\mathrm{CD}_{3} \mathrm{OD}, 4: 1\right) \delta 0.89$ (t, $J=7.2$ $\left.\mathrm{Hz}, 3 \mathrm{H}, \mathrm{CH}_{3}\right), 1.25-1.37$ (m, 4H, $\left.\mathrm{PCH}_{2} \mathrm{CHCHCH}_{2}\left(\mathrm{CH}_{2}\right)_{2} \mathrm{CH}_{3}\right)$, 1.98-2.07 (m, 2H, PCH2CHCHCH$\left.{ }_{2}\left(\mathrm{CH}_{2}\right)_{2} \mathrm{CH}_{3}\right), 2.61-2.68(\mathrm{~m}$, $\left.2 \mathrm{H}, \mathrm{PCH}_{2} \mathrm{NH}_{2}\right), 2.96-3.01\left(\mathrm{~m}, 2 \mathrm{H}, \mathrm{PCH} \mathrm{CHCH}_{2}\left(\mathrm{CH}_{2}\right)_{3} \mathrm{CH}_{3}\right), 5.07$ $\left(\mathrm{d}, J=8.7 \mathrm{~Hz}, 2 \mathrm{H}, \mathrm{OCH}_{2} \mathrm{Ph}\right), 5.28-5.39\left(\mathrm{~m}, 2 \mathrm{H}, \mathrm{PCH}_{2} \mathrm{CHCH}-\right.$ $\left.\left(\mathrm{CH}_{2}\right)_{3} \mathrm{CH}_{3}\right), 5.59-5.69\left(\mathrm{~m}, 2 \mathrm{H}, \mathrm{PCH}_{2} \mathrm{CHCH}\left(\mathrm{CH}_{2}\right)_{3} \mathrm{CH}_{3}\right), 7.32-$ 7.43 (m, 5H, Ph).

General Procedure for Amide Bond Formation. To solution of $N$-benzyloxycarbonyl-L-glutamyl( $\alpha$-benzylester)-L-leucine (300 $\mathrm{mg}, 0.619 \mathrm{mmol})$ and $N$-hydroxysuccinimide $(106.9 \mathrm{mg}, 0.923$ mmol) in anhydrous $N, N$-dimethylformamide $(5 \mathrm{~mL})$ cooled at 0 ${ }^{\circ} \mathrm{C}$ was added 1-(3-dimethylaminopropyl)-3-ethylcarbodiimide hydrochloride (EDC; $237.3 \mathrm{mg}, 2.24 \mathrm{mmol}$ ). The reaction mixture was stirred at room-temperature overnight. Then, a solution of corresponding amine $(0.495 \mathrm{mmol})$ and triethylamine $(15 \mu \mathrm{L}, 2.1$ mmol) in $N, N$-dimethylformamide $(3 \mathrm{~mL})$ was added, and the mixture was stirred at room temperature for $16 \mathrm{~h}$. Ethyl acetate $(20 \mathrm{~mL})$ was added, and the solution washed with brine $(2 \times 20$ $\mathrm{mL}), 5 \%$ aqueous hydrochloric acid $(2 \times 10 \mathrm{~mL})$, saturated sodium bicarbonate $(2 \times 10 \mathrm{~mL})$, and water $(2 \times 10 \mathrm{~mL})$. The organic layer was dried $\left(\mathrm{Na}_{2} \mathrm{SO}_{4}\right)$, and the solvent was evaporated. The product was purified by chromatography column (silica gel) using a mixture of hexanes-EtAcO (4:1) as eluant to yield the corresponding protected phosphinopeptide.

$O$-Benzyl Methyl-[[( $N$-benzyloxy-L- $\gamma$-glutamyl( $\alpha$-benzyl ester $)$ L-leucyl)amino]methyl]phosphinate (43). White solid, hygroscopic, $42 \%$ yield; ${ }^{1} \mathrm{H}$ NMR $\left(500.13 \mathrm{MHz}, \mathrm{CD}_{3} \mathrm{OD}\right) \delta 0.83-0.96$ $\left(\mathrm{m}, 6 \mathrm{H}, H_{3} \mathrm{C}(\delta) \mathrm{Leu}\right), 1.43-1.69\left(\mathrm{~m}, 6 \mathrm{H}, \mathrm{PCH}_{3}, H \mathrm{C}(\beta)\right.$ Leu, $H \mathrm{C}-$ $(\gamma)$ Leu $), 1.83-1.97\left(\mathrm{~m}, 1 \mathrm{H}, H_{\mathrm{a}} \mathrm{C}(\gamma) \mathrm{Glu}\right), 2.09-2.22\left(\mathrm{~m}, 1 \mathrm{H}, H_{\mathrm{b}} \mathrm{C}-\right.$ $(\gamma) \mathrm{Glu}), 3.58-3.76\left(\mathrm{~m}, 2 \mathrm{H}, \mathrm{NHCH}_{2} \mathrm{P}\right), 4.18-4.26(\mathrm{~m}, 1 \mathrm{H}, \mathrm{HC}-$ $(\alpha) \mathrm{Leu}), 4.30-4.38(\mathrm{~m}, 1 \mathrm{H}, H \mathrm{C}(\alpha) \mathrm{Glu}), 4.99-5.18(\mathrm{~m}, 6 \mathrm{H}$, $\left.\mathrm{OCH}_{2} \mathrm{Ph}\right), 7.22-7.41$ (m, 15H, Ph); ${ }^{13} \mathrm{C} \mathrm{NMR}\left(125.77 \mathrm{MHz}, \mathrm{CD}_{3}-\right.$ OD) $\delta 12.8$ (d, $J=93.3 \mathrm{~Hz}, \mathrm{PCH}_{3}$, major diast.), 12.9 (d, $J=$ $93.3 \mathrm{~Hz}, \mathrm{PCH}_{3}$, minor diast.), $21.9\left(\mathrm{H}_{3} C_{\mathrm{a}}(\delta)\right.$ Leu, minor diast.), 22.0 $\left(\mathrm{H}_{3} C_{\mathrm{a}}(\delta)\right.$ Leu, major diast. $), 23.3\left(\mathrm{H}_{3} C_{\mathrm{b}}(\delta)\right.$ Leu, minor diast. $), 23.3$ $\left(\mathrm{H}_{3} C_{\mathrm{b}}(\delta)\right.$ Leu, minor diast.), $25.9(\mathrm{C}(\gamma)$ Leu, minor diast $), 25.9(\mathrm{C}(\gamma)-$ Leu, major diast.), $28.3(\mathrm{C}(\gamma) \mathrm{Glu}), 32.7(\mathrm{C}(\beta) \mathrm{Glu}), 38.8(\mathrm{~d}, J=$ $102.6 \mathrm{~Hz}, \mathrm{NHCH}_{2} \mathrm{P}$, minor diast.), 38.9 (d, $J=102.6 \mathrm{~Hz}, \mathrm{NHCH}_{2} \mathrm{P}$, major diast.), $41.7(\mathrm{C}(\beta)$ Leu $), 53.3(\mathrm{C}(\alpha)$ Leu $), 55.1(\mathrm{C}(\alpha) \mathrm{Glu}), 67.6$ $\left(\mathrm{d}, J=5.9 \mathrm{~Hz}, \mathrm{POCH} \mathrm{H}_{2} \mathrm{Ph}\right), 67.7\left(\mathrm{OCH}_{2} \mathrm{Ph}\right), 68.0\left(\mathrm{OCH}_{2} \mathrm{Ph}\right), 128.8$ $(\mathrm{Ph}), 128.8(\mathrm{Ph}), 129.0(\mathrm{Ph}), 129.1(\mathrm{Ph}), 129.2(\mathrm{Ph}), 129.5(\mathrm{Ph})$, $129.5(\mathrm{Ph}), 129.6(\mathrm{Ph}), 129.7(\mathrm{Ph}), 129.7(\mathrm{Ph}), 172.6(C O), 175.2$ 
(CO), $175.6(C \mathrm{O}), 137.2(\mathrm{Ph}), 137.7$ (d, $J=6.8 \mathrm{~Hz}, \mathrm{Ph}), 138.1$ ( $\mathrm{Ph}), 158.6(\mathrm{CO}), 173.5$ (CO), 174.7 (CO), $175.0(\mathrm{CO}) ;{ }^{31} \mathrm{P}$ NMR (202.45 MHz, $\left.\mathrm{CDCl}_{3}\right) \delta 52.9$ (minor diast.), 53.1 (major diast.).

$O$-Benzyl Ethyl-[[( $N$-benzyloxy-L- $\gamma$-glutamyl( $\alpha$-benzyl ester)L-leucyl)amino]methyl]phosphinate (44). White solid, hygroscopic. $40 \%$ yield. White solid, hygroscopic. $40 \%$ yield.; ${ }^{1} \mathrm{H}$ NMR $\left(500.13 \mathrm{MHz}, \mathrm{CD}_{3} \mathrm{OD}\right) \delta 0.83-0.93\left(\mathrm{~m}, 9 \mathrm{H}, \mathrm{H}_{3} \mathrm{C}(\delta) \mathrm{Leu}, \mathrm{PCH}_{2} \mathrm{CH}_{3}\right)$, $1.01\left(\mathrm{dt}, J_{\mathrm{H}-\mathrm{P}}=18.8 \mathrm{~Hz}, J_{\mathrm{H}-\mathrm{H}}=7.6 \mathrm{~Hz}, 2 \mathrm{H}, \mathrm{PCH}_{2} \mathrm{CH}_{3}\right.$, minor diast.), 1.11 (dt, $J_{\mathrm{H}-\mathrm{P}}=18.8 \mathrm{~Hz}, J_{\mathrm{H}-\mathrm{H}}=7.6 \mathrm{~Hz}, 2 \mathrm{H}, \mathrm{PCH}_{2} \mathrm{CH}_{3}$, major diast.), $1.44-1.69$ (m, 3H, $H \mathrm{C}(\beta) \mathrm{Leu}, H \mathrm{C}(\gamma) \mathrm{Leu}), 1.73-$ $1.83\left(\mathrm{~m}, 2 \mathrm{H}, \mathrm{PCH}_{2} \mathrm{CH}_{3}\right), 1.83-1.95\left(\mathrm{~m}, 1 \mathrm{H}, H_{\mathrm{a}} \mathrm{C}(\gamma) \mathrm{Glu}\right), 2.07-$ $2.21\left(\mathrm{~m}, 1 \mathrm{H}, H_{\mathrm{b}} \mathrm{C}(\gamma) \mathrm{Glu}\right), 2.27-2.39(\mathrm{~m}, 2 \mathrm{H}, H \mathrm{C}(\beta) \mathrm{Glu}), 3.59-$ $3.77\left(\mathrm{~m}, 2 \mathrm{H}, \mathrm{NHCH}_{2} \mathrm{P}\right), 4.17-4.27(\mathrm{~m}, 1 \mathrm{H}, H \mathrm{C}(\alpha) \mathrm{Leu}), 4.27-$ $4.40(\mathrm{~m}, 1 \mathrm{H}, \mathrm{HC}(\alpha) \mathrm{Glu}), 4.98-5.19\left(\mathrm{~m}, 6 \mathrm{H}, \mathrm{OCH}_{2} \mathrm{Ph}\right), 7.23-7.42$ $(\mathrm{m}, 15 \mathrm{H}, \mathrm{Ph}) ;{ }^{13} \mathrm{C} \mathrm{NMR}\left(125.77 \mathrm{MHz}, \mathrm{CD}_{3} \mathrm{OD}\right) \delta 5.52(\mathrm{~d}, J=4.8$ $\mathrm{Hz}, \mathrm{PCH}_{2} \mathrm{CH}_{3}$, major diast.), 5.54 (d, $J=4.8 \mathrm{~Hz}, \mathrm{PCH}_{2} \mathrm{CH}_{3}$, minor diast.), 20.4 (d, $J=93.5 \mathrm{~Hz}, \mathrm{PCH}_{2} \mathrm{CH}_{3}$, major diast.), 20.6 (d, $J=$ $92.5 \mathrm{~Hz}, \mathrm{PCH}_{2} \mathrm{CH}_{3}$, minor diast.), $21.87\left(\mathrm{H}_{3} \mathrm{C}_{\mathrm{a}}(\delta) \mathrm{Leu}\right.$, minor diast.), $21.93\left(\mathrm{H}_{3} C_{\mathrm{a}}(\delta)\right.$ Leu, major diast. $), 23.35\left(\mathrm{H}_{3} C_{\mathrm{b}}(\delta)\right.$ Leu, minor diast. $)$, $23.40\left(\mathrm{H}_{3} C_{\mathrm{b}}(\delta)\right.$ Leu, minor diast.), $25.86(\mathrm{C}(\gamma)$ Leu, major diast), $25.89(\mathrm{C}(\gamma)$ Leu, minor diast.), $28.2(\mathrm{C}(\gamma) \mathrm{Glu}), 32.7(\mathrm{C}(\beta) \mathrm{Glu})$, 37.0 (d, $J=98.2 \mathrm{~Hz}, \mathrm{NHCH}_{2} \mathrm{P}$, major diast.), 37.1 (d, $J=98.2$ $\mathrm{Hz}, \mathrm{NHCH}_{2} \mathrm{P}$, minor diast.), $41.7(\mathrm{C}(\beta) \mathrm{Leu}), 53.2(\mathrm{C}(\alpha) \mathrm{Leu}), 55.1$ $(\mathrm{C}(\alpha) \mathrm{Glu}), 67.6\left(\mathrm{~d}, J=6.7 \mathrm{~Hz}, \mathrm{POCH}_{2} \mathrm{Ph}\right), 67.7\left(\mathrm{OCH}_{2} \mathrm{Ph}\right), 68.0$ $\left(\mathrm{OCH}_{2} \mathrm{Ph}\right), 128.8(\mathrm{Ph}), 129.0(\mathrm{Ph}), 129.2(\mathrm{Ph}), 129.3(\mathrm{Ph}), 129.5$ $(\mathrm{Ph}), 129.6(\mathrm{Ph}), 129.64(\mathrm{Ph}), 129.66(\mathrm{Ph}), 129.7(\mathrm{Ph}), 137.2(\mathrm{Ph})$, $137.9(\mathrm{~d}, J=5.7 \mathrm{~Hz}, \mathrm{Ph}), 138.1(\mathrm{Ph}), 158.7(C \mathrm{O}), 173.5(C \mathrm{O})$, 174.7 (CO), 175.0 (CO); ${ }^{31} \mathrm{P}$ NMR (202.45 MHz, $\left.\mathrm{CD}_{3} \mathrm{OD}\right) \delta 55.4$ (minor diast.), 55.6 (major diast.).

$O$-Benzyl 2-Propen-[[( $N$-benzyloxy-l- $\gamma$-glutamyl( $\alpha$-benzyl ester $)$ L-leucyl)amino]methyl]phosphinate (45). White solid, hygroscopic, $38 \%$ yield. ${ }^{1} \mathrm{H}$ NMR $\left(500.13 \mathrm{MHz}, \mathrm{CD}_{3} \mathrm{OD}\right) \delta 0.80-0.96$ $\left(\mathrm{m}, 6 \mathrm{H}, H_{3} \mathrm{C}(\delta)\right.$ Leu $), 1.45-1.57\left(\mathrm{~m}, 2 \mathrm{H}, H_{2} \mathrm{C}(\beta)\right.$ Leu $), 1.57-1.69$ $(\mathrm{m}, 1 \mathrm{H}, H \mathrm{C}(\gamma) \mathrm{Leu}), 1.83-1.94\left(\mathrm{~m}, 1 \mathrm{H}, H_{\mathrm{a}} \mathrm{C}(\beta) \mathrm{Glu}\right), 2.08-2.20$ (m, $\left.1 \mathrm{H}, H_{\mathrm{b}} \mathrm{C}(\beta) \mathrm{Glu}\right), 2.25-2.37(\mathrm{~m}, 2 \mathrm{H}, H \mathrm{C}(\gamma) \mathrm{Glu}), 2.61-2.78$ (m, 2H, $\left.\mathrm{PCH}_{2} \mathrm{CHCH}_{2}\right), 3.57-3.78\left(\mathrm{~m}, 2 \mathrm{H}, \mathrm{NHCH}_{2} \mathrm{P}\right), 4.13-4.27$ $(\mathrm{m}, 1 \mathrm{H}, H \mathrm{C}(\alpha) \mathrm{Leu}), 4.29-4.41(\mathrm{~m}, 1 \mathrm{H}, H \mathrm{C}(\alpha) \mathrm{Glu}), 4.95-5.31$ (m, 8H, OCH $\mathrm{OH}_{2} \mathrm{Ph} \mathrm{PCH}_{2} \mathrm{CHCH}_{2}$ ), 5.68-5.83 (m, 1H, $\mathrm{PCH}_{2} \mathrm{CHCH}_{2}$ ), 7.17-7.45 (m, 15H, Ph). ${ }^{13} \mathrm{C}$ NMR (125.77 MHz, $\left.\mathrm{CD}_{3} \mathrm{OD}\right) \delta 21.86$ $\left(\mathrm{H}_{3} C_{\mathrm{a}}(\delta)\right.$ Leu, major diast), $21.90\left(\mathrm{H}_{3} C_{\mathrm{a}}(\delta)\right.$ Leu, minor diast), 23.36 $\left(\mathrm{H}_{3} C_{\mathrm{b}}(\delta)\right.$ Leu $), 25.85(\mathrm{C}(\gamma)$ Leu, minor diast.), $25.89(\mathrm{C}(\gamma)$ Leu, major diast.), $26.3\left(\mathrm{PCH}_{2} \mathrm{CHCH}_{2}\right), 28.2(\mathrm{C}(\gamma) \mathrm{Glu}), 32.7(\mathrm{C}(\beta) \mathrm{Glu}), 33.6$ (d, $J=88.0 \mathrm{~Hz}, \mathrm{PCH}_{2} \mathrm{CHCH}_{2}$, minor diast.), 33.7 (d, $J=88.0$ $\mathrm{Hz}, \mathrm{PCH}_{2} \mathrm{CHCH}_{2}$, major diast.), 37.3 (d, $\left.J=100.5 \mathrm{~Hz}, \mathrm{NHCH}_{2} \mathrm{P}\right)$, $41.7(\mathrm{C}(\beta) \mathrm{Leu}), 53.2(\mathrm{C}(\alpha) \mathrm{Leu}), 55.1(\mathrm{C}(\alpha) \mathrm{Glu}), 67.7\left(\mathrm{OCH}_{2} \mathrm{Ph}\right)$, $67.9\left(\mathrm{~d}, J=7.2 \mathrm{~Hz}, \mathrm{OCH}_{2} \mathrm{Ph}\right), 67.9\left(\mathrm{OCH}_{2} \mathrm{Ph}\right), 121.6(\mathrm{~d}, J=12.6$ $\mathrm{Hz}, \mathrm{PCH}_{2} \mathrm{CHCH}_{2}$ ), 127.6 (d, $J=9.9 \mathrm{~Hz}, \mathrm{PCH}_{2} \mathrm{CHCH}_{2}$, minor diast.), 127.7 (d, $J=9.9 \mathrm{~Hz}, \mathrm{PCH}_{2} \mathrm{CHCH}_{2}$, major diast.), 128.3 $(\mathrm{Ph}), 129.0(\mathrm{Ph}), 129.2(\mathrm{Ph}), 129.3(\mathrm{Ph}), 129.5(\mathrm{Ph}), 129.56(\mathrm{Ph})$ $129.62(\mathrm{Ph}), 137.2(\mathrm{Ph}), 137.8(\mathrm{~d}, J=7.2 \mathrm{~Hz}, \mathrm{Ph}), 138.1(\mathrm{Ph})$, 158.7 (CO), 173.5 (CO), 174.7 (CO), 174.9 (d, $J=12.6 \mathrm{~Hz}, \mathrm{CO}$ ); ${ }^{31} \mathrm{P}$ NMR (202.45 MHz, $\left.\mathrm{CD}_{3} \mathrm{OD}\right) \delta 49.3$ (major diast.), 49.4 (minor diast.).

$O$-Benzyl 2-Butyl-[[( $N$-benzyloxy-L- $\gamma$-glutamyl( $\alpha$-benzyl ester)L-leucyl)amino]methyl]phosphinate (46). White solid, hygroscopic. 39\% yield; ${ }^{1} \mathrm{H}$ NMR $\left(500.13 \mathrm{MHz}, \mathrm{CD}_{3} \mathrm{OD}\right) \delta 0.81-0.94$ (m, 9H, $\mathrm{H}_{3} \mathrm{C}(\delta)$ Leu, $\left.\mathrm{P}\left(\mathrm{CH}_{2}\right)_{3} \mathrm{CH}_{3}\right), 1.30-1.42\left(\mathrm{~m}, 2 \mathrm{H}, \mathrm{P}\left(\mathrm{CH}_{2}\right)_{2} \mathrm{CH}_{2-}\right.$ $\left.\mathrm{CH}_{3}\right), 1.44-0.1 .68\left(\mathrm{~m}, 5 \mathrm{H}, \mathrm{PCH}_{2} \mathrm{CH}_{2} \mathrm{CH}_{2} \mathrm{CH}_{3}, \mathrm{H}_{2} \mathrm{C}(\beta) \mathrm{Leu}, \mathrm{HC}-\right.$ $(\gamma)$ Leu $), 1.71-1.84\left(\mathrm{~m}, 2 \mathrm{H}, \mathrm{PCH}_{2}\left(\mathrm{CH}_{2}\right)_{2} \mathrm{CH}_{3}\right), 1.84-1.95(\mathrm{~m}, 1 \mathrm{H}$, $\left.H_{\mathrm{a}} \mathrm{C}(\gamma) \mathrm{Glu}\right), 2.08-2.22\left(\mathrm{~m}, 1 \mathrm{H}, H_{\mathrm{b}} \mathrm{C}(\gamma) \mathrm{Glu}\right), 2.27-2.39(\mathrm{~m}, 2 \mathrm{H}$, $\left.\mathrm{H}_{2} \mathrm{C}(\gamma) \mathrm{Glu}\right), 3.58-3.76\left(\mathrm{~m}, 2 \mathrm{H}, \mathrm{NHCH}_{2} \mathrm{P}\right), 4.15-4.26(\mathrm{~m}, 1 \mathrm{H}, \mathrm{HC}-$ $(\alpha) \mathrm{Leu}), 4.30-4.7(\mathrm{~m}, 1 \mathrm{H}, \mathrm{HC}(\alpha) \mathrm{Glu}), 4.99-5.12\left(\mathrm{~m}, 4 \mathrm{H}, \mathrm{OCH}_{2-}\right.$ $\mathrm{Ph}), 5.14$ (s, 2H, OCH$\left.H_{2} \mathrm{Ph}\right), 7.23-7.42(\mathrm{~m}, 15 \mathrm{H}, \mathrm{Ph}) ;{ }^{13} \mathrm{C}$ NMR

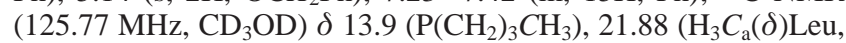
minor diast.), $21.94\left(\mathrm{H}_{3} C_{\mathrm{a}}(\delta)\right.$ Leu, major diast. $), 23.32\left(\mathrm{H}_{3} C_{\mathrm{b}}(\delta)\right.$ Leu, major diast.), 23.37 ( $\mathrm{H}_{3} C_{\mathrm{b}}(\delta)$ Leu, minor diast.), 24.3 (d, $J=$ $\left.4.2 \mathrm{~Hz}, \mathrm{P}\left(\mathrm{CH}_{2}\right)_{2} \mathrm{CH}_{2} \mathrm{CH}_{3}\right), 24.8\left(\mathrm{~d}, J=14.4 \mathrm{~Hz}, \mathrm{PCH}_{2} \mathrm{CH}_{2} \mathrm{CH}_{2}-\right.$ $\left.\mathrm{CH}_{3}\right), 25.84(\mathrm{C}(\gamma)$ Leu, major diast $), 25.89(\mathrm{C}(\gamma)$ Leu, minor diast. $)$, 27.0 (d, $J=91.6 \mathrm{~Hz}, \mathrm{PCH}_{2} \mathrm{NH}$, minor diast.), 27.2 (d, $J=91.6$ $\mathrm{Hz}, \mathrm{PCH}_{2} \mathrm{NH}$, major diast. $), 28.2(\mathrm{C}(\gamma) \mathrm{Glu}), 32.7(\mathrm{C}(\beta) \mathrm{Glu}), 37.5$ (d, $J=98.3 \mathrm{~Hz}, \mathrm{NHCH}_{2} \mathrm{P}$, minor diast.), $37.6(\mathrm{~d}, J=98.3 \mathrm{~Hz}$,
$\mathrm{NHCH}_{2} \mathrm{P}$, major diast.), $41.7(\mathrm{C}(\beta) \mathrm{Leu}), 53.2(\mathrm{C}(\alpha) \mathrm{Leu}), 55.1$ $(\mathrm{C}(\alpha) \mathrm{Glu}), 67.57$ (d, $J=5.9 \mathrm{~Hz}, \mathrm{POCH}_{2} \mathrm{Ph}$, major diast.), 67.59 (d, $J=5.9 \mathrm{~Hz}, \mathrm{POCH}_{2} \mathrm{Ph}$, minor diast.), $67.7\left(\mathrm{OCH}_{2} \mathrm{Ph}\right), 68.0$ $\left(\mathrm{OCH}_{2} \mathrm{Ph}\right), 128.8(\mathrm{Ph}), 129.0(\mathrm{Ph}), 129.1(\mathrm{Ph}), 129.2(\mathrm{Ph}), 129.3-$ $(\mathrm{Ph}), 129.48(\mathrm{Ph}), 129.51(\mathrm{Ph}), 129.57(\mathrm{Ph}), 129.64(\mathrm{Ph}), 129.66$ $(\mathrm{Ph}), 137.88$ (d, $J=5.9 \mathrm{~Hz}, \mathrm{Ph}$, minor diast.), 137.89 (d, $J=5.9$ $\mathrm{Hz}, \mathrm{Ph}$, major diast.), 138.1 ( $\mathrm{Ph}), 158.7$ (CO), 173.5 (CO), 174.7 (CO), 174.9 (d, $J=11.0 \mathrm{~Hz}, C \mathrm{O}) ;{ }^{31} \mathrm{P}$ NMR $\left(202.45 \mathrm{MHz}, \mathrm{CDCl}_{3}\right)$ $\delta 54.4$ (minor diast.), 54.5 (major diast.).

$O$-Benzyl 2-Penten-[[( $N$-benzyloxy-L- $\gamma$-glutamyl( $\alpha$-benzyl ester)L-leucyl)amino]methyl]phosphinate (47). White solid, hygroscopic. $36 \%$ yield. ${ }^{1} \mathrm{H}$ NMR $\left(500.13 \mathrm{MHz}, \mathrm{CD}_{3} \mathrm{OD}\right) \delta 0.82-1.00$ $\left(\mathrm{m}, 9 \mathrm{H}, \mathrm{H}_{3} \mathrm{C}(\delta)\right.$ Leu, $\left.\mathrm{PCH}_{2} \mathrm{CHCHCH}_{2} \mathrm{CH}_{3}\right), 1.47-1.57(\mathrm{~m}, 2 \mathrm{H}$, $\left.\left.\mathrm{H}_{2} \mathrm{C}(\beta) \mathrm{Leu}\right), 1.57-1.69 \mathrm{~m}, 1 \mathrm{H}, H \mathrm{C}(\gamma) \mathrm{Leu}\right), 1.83-1.95\left(\mathrm{~m}, 1 \mathrm{H}, H_{\mathrm{a}} \mathrm{C}-\right.$ $(\beta) \mathrm{Glu}), 1.97-2.07\left(2 \mathrm{H}, 2.09-2.21, \mathrm{PCH}_{2} \mathrm{CHCHCH}_{2} \mathrm{CH}_{3}\right), 2.10-$ $2.20\left(\mathrm{~m}, 1 \mathrm{H}, H_{\mathrm{b}} \mathrm{C}(\beta) \mathrm{Glu}\right), 2.28-2.39\left(\mathrm{~m}, 2 \mathrm{H}, H_{\mathrm{b}} \mathrm{C}(\gamma) \mathrm{Glu}\right), 2.58-$ $2.68\left(\mathrm{~m}, 2 \mathrm{H}, \mathrm{PCH}_{2} \mathrm{CHCHCH}_{2} \mathrm{CH}_{3}\right), 3.59-3.76\left(\mathrm{~m}, 2 \mathrm{H}, \mathrm{NHCH}_{2} \mathrm{P}\right)$, 4.19-4.28 (m, 1H, $H \mathrm{C}(\alpha) \mathrm{Leu}), 4.33-4.51(\mathrm{~m}, 1 \mathrm{H}, H \mathrm{C}(\alpha) \mathrm{Glu})$, $5.99-5.19\left(\mathrm{~m}, 6 \mathrm{H}, \mathrm{OCH}_{2} \mathrm{Ph}\right), 5.31-5.41\left(\mathrm{~m}, 1 \mathrm{H}, \mathrm{PCH}_{2} \mathrm{CHCHCH}_{2-}\right.$ $\left.\mathrm{CH}_{3}\right), 5.58-5.68\left(\mathrm{~m}, 1 \mathrm{H}, \mathrm{PCH}_{2} \mathrm{CHCHCH}_{2} \mathrm{CH}_{3}\right), 7.22-7.40(\mathrm{~m}$, $15 \mathrm{H}, \mathrm{Ph}) ;{ }^{13} \mathrm{C}$ NMR $\left(125.77 \mathrm{MHz}, \mathrm{CD}_{3} \mathrm{OD}\right) \delta 13.79\left(\mathrm{PCH}_{2^{-}}\right.$ $\mathrm{CHCHCH}_{2} \mathrm{CH}_{3}$, minor diast.), $13.82\left(\mathrm{PCH}_{2} \mathrm{CHCHCH}_{2} \mathrm{CH}_{3}\right.$, major diast.), $21.90\left(\mathrm{H}_{3} C_{\mathrm{a}}(\delta)\right.$ Leu, minor diast $), 21.93\left(\mathrm{H}_{3} C_{\mathrm{a}}(\delta)\right.$ Leu, major diast), $23.38\left(\mathrm{H}_{3} C_{\mathrm{b}}(\delta)\right.$ Leu, minor diast. $), 23.41\left(\mathrm{H}_{3} C_{\mathrm{b}}(\delta)\right.$ Leu, major diast.), $25.8(\mathrm{C}(\gamma)$ Leu, minor diast.), $25.9(\mathrm{C}(\gamma)$ Leu, major diast.), $26.7\left(\mathrm{PCH}_{2} \mathrm{CHCHCH}_{2} \mathrm{CH}_{3}\right.$, minor diast.), $26.8\left(\mathrm{PCH}_{2} \mathrm{CHCHCH}_{2}-\right.$ $\mathrm{CH}_{3}$, major diast.), $28.2(\mathrm{C}(\gamma) \mathrm{Glu}), 32.1\left(\mathrm{~d}, J=89.0 \mathrm{~Hz}, \mathrm{PCH}_{2^{-}}\right.$ $\mathrm{CHCHCH}_{2} \mathrm{CH}_{3}$, minor diast.), 32.3 (d, $J=88.3 \mathrm{~Hz}, \mathrm{PCH}_{2} \mathrm{CHCHCH}_{2}-$ $\mathrm{CH}_{3}$, major diast.), $32.7(\mathrm{C}(\beta) \mathrm{Glu}), 37.2(\mathrm{~d}, J=100.0 \mathrm{~Hz}$, $\mathrm{NHCH}_{2} \mathrm{P}$, minor diast.), $37.3\left(\mathrm{~d}, J=100.0 \mathrm{~Hz}, \mathrm{NHCH}_{2} \mathrm{P}\right.$, major diast.), $41.7(\mathrm{C}(\beta)$ Leu $), 53.2(\mathrm{C}(\alpha)$ Leu $), 55.1(\mathrm{C}(\alpha) \mathrm{Glu}), 67.7$ (O$\left.\mathrm{CH}_{2}-\mathrm{Ph}\right), 67.8\left(\mathrm{~d}, J=6.8 \mathrm{~Hz}, \mathrm{OCH}_{2} \mathrm{Ph}\right), 68.0\left(\mathrm{OCH}_{2} \mathrm{Ph}\right), 117.4$ (d, $J=10.2 \mathrm{~Hz}, \mathrm{PCH}_{2} \mathrm{CHCHCH}_{2} \mathrm{CH}_{3}$, major diast.), 117.5 (d, $J=$ $10.2 \mathrm{~Hz}, \mathrm{PCH}_{2} \mathrm{CHCHCH}_{2} \mathrm{CH}_{3}$, major diast.), 128.96 (CO), 129.0 (CO), 129.2 (CO), 129.3 (CO), 129.5 (CO), 129.6 (CO), 129.61 (CO), $129.62(\mathrm{CO}), 137.2(\mathrm{Ph}), 137.87(\mathrm{~d}, J=5.9 \mathrm{~Hz}, \mathrm{Ph}$, major diast.), 137.89 (d, $J=5.9 \mathrm{~Hz}, \mathrm{Ph}$, major diast.), $138.1(\mathrm{Ph}), 140.1$ (d, $\left.J=12.7 \mathrm{~Hz}, \mathrm{PCH}_{2} \mathrm{CHCHCH}_{2} \mathrm{CH}_{3}\right), 158.65$ (CO, minor diast.), 158.67 (CO, major diast.), 173,5 (CO), 174.40 (CO, major diast.), 174.73 (CO, minor diast.), 174.85 (CO, minor diast), 174.88 (CO, major diast.), 174.5 (CO); ${ }^{31} \mathrm{P}$ NMR (202.45 MHz, $\left.\mathrm{CD}_{3} \mathrm{OD}\right) \delta 50.1$ (major diast.), 50.2 (minor diast.).

$O$-Benzyl 2-Hexen-[[( $N$-benzyloxy-L- $\gamma$-glutamyl( $\alpha$-benzyl ester)L-leucyl)amino]methyl]phosphinate $\mathbf{( 4 8 )}$. White solid, hygroscopic, 63\% yield; ${ }^{1} \mathrm{H}$ NMR $\left(500.13 \mathrm{MHz}, \mathrm{CD}_{3} \mathrm{OD}\right) \delta 0.83-0.93$ $\left(\mathrm{m}, 9 \mathrm{H}, \mathrm{H}_{3} \mathrm{C}(\delta) \mathrm{Leu}, \mathrm{PCH}_{2} \mathrm{CHCH}\left(\mathrm{CH}_{2}\right)_{2} \mathrm{CH}_{3}\right), 1.30-1.41(\mathrm{~m}, 2 \mathrm{H}$, $\left.\mathrm{PCH}_{2} \mathrm{CHCHCH}_{2} \mathrm{CH}_{2} \mathrm{CH}_{3}\right), 1.48-1.57\left(\mathrm{~m}, 2 \mathrm{H}, \mathrm{H}_{2} \mathrm{C}(\beta) \mathrm{Leu}\right), 1.57-$ $1.68(\mathrm{~m}, 1 \mathrm{H}, H \mathrm{C}(\gamma) \mathrm{Leu}), 1.85-2.02\left(\mathrm{~m}, 3 \mathrm{H}, H_{\mathrm{a}} \mathrm{C}(\beta) \mathrm{Glu}, \mathrm{PCH}_{2^{-}}\right.$ $\left.\mathrm{CHCHCH}_{2} \mathrm{CH}_{2} \mathrm{CH}_{3}\right), 2.09-2.21\left(\mathrm{~m}, 1 \mathrm{H}, H_{\mathrm{b}} \mathrm{C}(\beta) \mathrm{Glu}\right), 2.29-2.37$ (m, $\left.2 \mathrm{H}, H_{\mathrm{b}} \mathrm{C}(\gamma) \mathrm{Glu}\right), 2.57-2.68\left(\mathrm{~m}, 2 \mathrm{H}, \mathrm{PCH} \mathrm{CHCH}_{2}\left(\mathrm{CH}_{2}\right)_{2} \mathrm{CH}_{3}\right)$, $3.58-3.74\left(\mathrm{~m}, 2 \mathrm{H}, \mathrm{NHCH}_{2} \mathrm{P}\right), 4.19-4.27(\mathrm{~m}, 1 \mathrm{H}, H \mathrm{C}(\alpha) \mathrm{Leu})$, 4.33-4.40 (m, $1 \mathrm{H}, H \mathrm{C}(\alpha) \mathrm{Glu}), 5.99-5.17\left(\mathrm{~m}, 6 \mathrm{H}, \mathrm{OCH}_{2} \mathrm{Ph}\right)$, 5.31-5.41 (m, 1H, $\left.\mathrm{PCH}_{2} \mathrm{CHCH}\left(\mathrm{CH}_{2}\right)_{2} \mathrm{CH}_{3}\right), 5.58-5.69(\mathrm{~m}, 1 \mathrm{H}$, $\left.\mathrm{PCH}_{2} \mathrm{CHCH}\left(\mathrm{CH}_{2}\right)_{2} \mathrm{CH}_{3}\right), 7.21-7.40(\mathrm{~m}, 15 \mathrm{H}, \mathrm{Ph}) ;{ }^{13} \mathrm{C} \mathrm{NMR}$ $\left(125.77 \mathrm{MHz}, \mathrm{CD}_{3} \mathrm{OD}\right) \delta 13.79\left(\mathrm{PCH}_{2} \mathrm{CHCH}\left(\mathrm{CH}_{2}\right)_{2} \mathrm{CH}_{3}\right), 21.93$ $\left(\mathrm{H}_{3} C_{\mathrm{a}}(\delta)\right.$ Leu, minor diast), $21.97\left(\mathrm{H}_{3} C_{\mathrm{a}}(\delta)\right.$ Leu, major diast $), 23.29$ $\left(\mathrm{H}_{3} C_{\mathrm{b}}(\delta)\right.$ Leu, minor diast.), $23.31\left(\mathrm{H}_{3} C_{\mathrm{b}}(\delta)\right.$ Leu, major diast. $), 23.4$ $\left(\mathrm{d}, J=2.5 \mathrm{~Hz}, \mathrm{PCH}_{2} \mathrm{CHCHCH}_{2} \mathrm{CH}_{2} \mathrm{CH}_{3}\right), 25.88(\mathrm{C}(\gamma)$ Leu, major diast.), 25.91 ( $\mathrm{C}(\gamma)$ Leu, minor diast.), 28.2 (C( $\gamma) \mathrm{Glu}), 31.9$ (d, $J$ $=89.0 \mathrm{~Hz}, \mathrm{PCH}_{2} \mathrm{CHCH}\left(\mathrm{CH}_{2}\right)_{2} \mathrm{CH}_{3}$, major diast.), $32.3(\mathrm{~d}, J=89.0$ $\mathrm{Hz}, \mathrm{PCH}_{2} \mathrm{CHCH}\left(\mathrm{CH}_{2}\right)_{2} \mathrm{CH}_{3}$, minor diast.), $32.7(\mathrm{C}(\beta) \mathrm{Glu}), 35.8$ $\left(\mathrm{d}, J=2.5 \mathrm{~Hz}, \mathrm{PCH}_{2} \mathrm{CHCHCH}_{2} \mathrm{CH}_{2} \mathrm{CH}_{3}\right), 37.31$ (d, $J=100.0$ $\mathrm{Hz}, \mathrm{NHCH}_{2} \mathrm{P}$, major diast.), 37.33 (d, $J=100.0 \mathrm{~Hz}, \mathrm{NHCH}_{2} \mathrm{P}$, major diast.), $41.7(\mathrm{C}(\beta)$ Leu $), 53.2(\mathrm{C}(\alpha)$ Leu $), 55.1(\mathrm{C}(\alpha) \mathrm{Glu}), 67.7$ $\left(\mathrm{OCH}_{2} \mathrm{Ph}\right), 67.8\left(\mathrm{~d}, J=6.8 \mathrm{~Hz}, \mathrm{OCH}_{2} \mathrm{Ph}\right), 68.0\left(\mathrm{OCH}_{2} \mathrm{Ph}\right), 118.5$ (d, $J=9.3 \mathrm{~Hz}, \mathrm{PCH}_{2} \mathrm{CHCH}\left(\mathrm{CH}_{2}\right)_{2} \mathrm{CH}_{3}$, major diast.), 118.6 (d, $J$ $=10.2 \mathrm{~Hz}, \mathrm{PCH}_{2} \mathrm{CHCH}\left(\mathrm{CH}_{2}\right)_{2} \mathrm{CH}_{3}$, major diast.), $128.8(\mathrm{Ph})$, $128.98(\mathrm{Ph}), 129.03(\mathrm{Ph}), 129.14(\mathrm{Ph}), 129.3(\mathrm{Ph}), 129.45(\mathrm{Ph})$, 129.48 (Ph), 129.57 (Ph), 129.61 (Ph), 129.63 (Ph) 137.2 (Ph), 137.9 $(\mathrm{d}, J=5.9 \mathrm{~Hz}, \mathrm{Ph}), 138.1(\mathrm{Ph}), 138.5\left(\mathrm{~d}, J=12.7 \mathrm{~Hz}, \mathrm{PCH}_{2^{-}}\right.$ $\left.\mathrm{CHCH}\left(\mathrm{CH}_{2}\right)_{2} \mathrm{CH}_{3}\right), 158.7$ (CO), 173,5 (CO), 173.5 (CO), 174.7 
(CO), 174.9 (d, $J=11.9 \mathrm{~Hz}, \mathrm{CO}) ;{ }^{31} \mathrm{P}$ NMR (202.45 MHz, $\mathrm{CD}_{3}$ OD) $\delta 49.9$ (major diast.), 50.1 (minor diast.).

$O$-Benzyl 2-Hepten-[[( $N$-benzyloxy-L- $\gamma$-glutamyl $(\alpha$-benzyl ester)-L-leucyl)amino]methyl]phosphinate (49). White solid, hygroscopic, $56 \%$ yield; ${ }^{1} \mathrm{H}$ NMR $\left(500.13 \mathrm{MHz}, \mathrm{CD}_{3} \mathrm{OD}\right) \delta 0.75-$ $0.96\left(\mathrm{~m}, 9 \mathrm{H}, \mathrm{H}_{3} \mathrm{C}(\delta) \mathrm{Leu}, \mathrm{PCH}_{2} \mathrm{CHCHCH}_{2}\left(\mathrm{CH}_{2}\right)_{2} \mathrm{CH}_{3}\right) 1.18-1.36$ $\left(\mathrm{m}, 4 \mathrm{H}, \mathrm{PCH}_{2} \mathrm{CHCHCH}_{2}\left(\mathrm{CH}_{2}\right)_{2} \mathrm{CH}_{3}\right), 1.43-1.56\left(\mathrm{~m}, 2 \mathrm{H}, \mathrm{H}_{2} \mathrm{C}(\beta)-\right.$ Leu), 1.56-1.68 (m, 1H, $H \mathrm{C}(\gamma)$ Leu $), 1.81-2.05\left(\mathrm{~m}, 3 \mathrm{H}, H_{\mathrm{a}} \mathrm{C}(\beta) \mathrm{Glu}\right.$, $\left.\mathrm{PCH}_{2} \mathrm{CHCHCH}_{2}\left(\mathrm{CH}_{2}\right)_{2} \mathrm{CH}_{3}\right), 2.07-2.21\left(\mathrm{~m}, 1 \mathrm{H}, H_{\mathrm{b}} \mathrm{C}(\beta) \mathrm{Glu}\right)$, 2.24-2.38 (m, 2H, $\left.H_{\mathrm{b}} \mathrm{C}(\gamma) \mathrm{Glu}\right), 2.53-2.69\left(\mathrm{~m}, 2 \mathrm{H}, \mathrm{PCH}_{2^{-}}\right.$ $\left.\mathrm{CHCHCH}_{2}\left(\mathrm{CH}_{2}\right)_{2} \mathrm{CH}_{3}\right), 3.55-3.75\left(\mathrm{~m}, 2 \mathrm{H}, \mathrm{NHCH}_{2} \mathrm{P}\right), 4.15-4.27$ $(\mathrm{m}, 1 \mathrm{H}, H \mathrm{C}(\alpha) \mathrm{Leu}), 4.29-4.40(\mathrm{~m}, 1 \mathrm{H}, H \mathrm{C}(\alpha) \mathrm{Glu}), 5.91-5.17$ $\left(\mathrm{m}, 6 \mathrm{H}, \mathrm{OCH}_{2} \mathrm{Ph}\right), 5.27-5.40\left(\mathrm{~m}, 1 \mathrm{H}, \mathrm{PCH}_{2} \mathrm{CHCH}\left(\mathrm{CH}_{2}\right)_{3} \mathrm{CH}_{3}\right)$, 5.56-5.69 (m, $\left.1 \mathrm{H}, \mathrm{PCH}_{2} \mathrm{CHCH}\left(\mathrm{CH}_{2}\right)_{3} \mathrm{CH}_{3}\right), 7.18-7.41(\mathrm{~m}, 15 \mathrm{H}$, $\mathrm{Ph}) ;{ }^{13} \mathrm{C}$ NMR (125.77 MHz, $\left.\mathrm{CD}_{3} \mathrm{OD}\right) \delta 14.2\left(\mathrm{PCH}_{2} \mathrm{CHCH}-\right.$ $\left.\left(\mathrm{CH}_{2}\right)_{3} \mathrm{CH}_{3}\right), 21.96\left(\mathrm{H}_{3} C_{\mathrm{a}}(\delta)\right.$ Leu, major diast), $22.0\left(\mathrm{H}_{3} C_{\mathrm{a}}(\delta)\right.$ Leu, minor diast), $23.2\left(\mathrm{PCH}_{2} \mathrm{CHCH}\left(\mathrm{CH}_{2}\right)_{2} \mathrm{CH}_{2} \mathrm{CH}_{3}\right), 23.38\left(\mathrm{H}_{3} C_{\mathrm{b}}(\delta)-\right.$ Leu, major diast), $23.41\left(\mathrm{H}_{3} C_{\mathrm{b}}(\delta) \mathrm{Leu}\right.$, minor diast $), 25.88(\mathrm{C}(\gamma)$ Leu, major diast.), 25.91 (C( $\gamma)$ Leu, minor diast.), 28.3 (C $(\gamma)$ Glu), 32.2 (d, $J=88.1 \mathrm{~Hz}, \mathrm{PCH}_{2} \mathrm{CHCH}\left(\mathrm{CH}_{2}\right)_{3} \mathrm{CH}_{3}$, major diast.), 32.4 (d, $J=88.1 \mathrm{~Hz}, \mathrm{PCH}_{2} \mathrm{CHCH}\left(\mathrm{CH}_{2}\right)_{3} \mathrm{CH}_{3}$, minor diast.), 32.4 (d, $J$ $\left.=3.4 \mathrm{~Hz}, \mathrm{PCH}_{2} \mathrm{CHCHCH}_{2} \mathrm{CH}_{2} \mathrm{CH}_{2} \mathrm{CH}_{3}\right), 32.7(\mathrm{C}(\beta) \mathrm{Glu}), 33.4$ $\left(\mathrm{d}, J=2.5 \mathrm{~Hz}, \mathrm{PCH}_{2} \mathrm{CHCHCH}_{2}\left(\mathrm{CH}_{2}\right)_{2} \mathrm{CH}_{3}\right), 37.3(\mathrm{~d}, J=99.2$ $\left.\mathrm{Hz}, \mathrm{NHCH}_{2} \mathrm{P}\right), 41.7(\mathrm{C}(\beta) \mathrm{Leu}), 53.2(\mathrm{C}(\alpha) \mathrm{Leu}), 55.1(\mathrm{C}(\alpha) \mathrm{Glu})$, $67.7\left(\mathrm{OCH}_{2} \mathrm{Ph}\right), 67.8\left(\mathrm{~d}, J=6.8 \mathrm{~Hz}, \mathrm{OCH}_{2} \mathrm{Ph}\right), 68.0\left(\mathrm{OCH}_{2} \mathrm{Ph}\right)$, 118.36 (d, $J=9.3 \mathrm{~Hz}, \mathrm{PCH}_{2} \mathrm{CHCH}\left(\mathrm{CH}_{2}\right)_{3} \mathrm{CH}_{3}$, major diast.), 118.41 (d, $J=10.2 \mathrm{~Hz}, \mathrm{PCH}_{2} \mathrm{CHCH}\left(\mathrm{CH}_{2}\right)_{3} \mathrm{CH}_{3}$, major diast.), $128.8(\mathrm{Ph})$, $128.96(\mathrm{Ph}), 129.03(\mathrm{Ph}), 129.14(\mathrm{Ph}), 129.3(\mathrm{Ph}), 129.45(\mathrm{Ph})$, $129.49(\mathrm{Ph}), 129.57(\mathrm{Ph}), 129.62(\mathrm{Ph}), 129.64(\mathrm{Ph}), 137.2(\mathrm{Ph})$, $137.9(\mathrm{~d}, J=5.9 \mathrm{~Hz}, \mathrm{Ph}), 138.1(\mathrm{Ph}), 138.7\left(\mathrm{~d}, J=12.7 \mathrm{~Hz}, \mathrm{PCH}_{2^{-}}\right.$ $\left.\mathrm{CHCH}\left(\mathrm{CH}_{2}\right)_{3} \mathrm{CH}_{3}\right), 158.6(\mathrm{CO}), 173.5(\mathrm{CO}), 173.5(\mathrm{CO}), 174.7$ (CO), 174.9 (d, $J=9.3 \mathrm{~Hz}, \mathrm{CO}) ;{ }^{31} \mathrm{P}$ NMR $\left(202.45 \mathrm{MHz}, \mathrm{CD}_{3^{-}}\right.$ OD) $\delta 49.9$ (minor diast.), 50.0 (major diast.).

General Procedure for Benzyl Ether Cleavage and Alkene Hydrogenation. A solution of the respective protected phosphinopeptide $(0.1 \mathrm{mmol})$ in methanol $(10 \mathrm{~mL})$ in the presence of palladium on charcoal (catalyst) was treated with hydrogen at 1 atm. The reaction mixture was stirred at room temperature for $1 \mathrm{~h}$. The mixture was filtered through a glass frit filter, and the solvent was evaporated. The residue was purified by high performance liquid chromatography employing a Beckman Ultrasphere ODS-2 $5 \mu \mathrm{M}$ column $(10 \mathrm{~mm} \times 250 \mathrm{~mm})$ at a rate of $3 \mathrm{~mL} / \mathrm{min}$. Eluent solvents and retention times are indicated in each case. Peak elution was monitored at $230 \mathrm{~nm}$.

Methyl-[[(L- $\gamma$-glutamyl-L-leucyl)amino]methyl]phosphinate (50). HPLC purification: eluent solvent $\mathrm{H}_{2} \mathrm{O}-\mathrm{MeCN}-\mathrm{TFA}$ (90:10:0.2), $t_{\mathrm{R}}=10.45 \mathrm{~min}$ : white solid; $100 \%$ yield; ${ }^{1} \mathrm{H}$ NMR $(500.13 \mathrm{MHz}$, $\left.\mathrm{D}_{2} \mathrm{O}\right) \delta 0.97\left(\mathrm{~d}, J=6.1 \mathrm{~Hz}, 3 \mathrm{H}, H_{3} \mathrm{C}_{\mathrm{a}}(\delta)\right.$ Leu $), 1.01(\mathrm{~d}, J=6.4$ $\left.\mathrm{Hz}, 3 \mathrm{H}, H_{3} \mathrm{C}_{\mathrm{b}}(\delta) \mathrm{Leu}\right), 1.49\left(\mathrm{~d}, J=13.7 \mathrm{~Hz}, 3 \mathrm{H}, \mathrm{P}-\mathrm{CH}_{3}\right), 1.62-$ $1.78\left(\mathrm{~m}, 3 \mathrm{H}, H_{2} \mathrm{C}(\beta)\right.$ Leu, $H \mathrm{C}(\gamma)$ Leu $), 2.20-2.36\left(\mathrm{~m}, 2 \mathrm{H}, H_{2} \mathrm{C}(\beta)-\right.$ Glu), $2.57-2.70\left(\mathrm{~m}, 2 \mathrm{H}, \mathrm{H}_{2} \mathrm{C}(\gamma) \mathrm{Glu}\right), 3.48\left(\mathrm{dd}, J_{\mathrm{H}-\mathrm{H}}=15.5, J_{\mathrm{P}-\mathrm{H}}\right.$ $=9.6 \mathrm{~Hz}, 2 \mathrm{H}, \mathrm{NH}-\mathrm{CH}_{a}-\mathrm{P}$, minor diasteromer $), 3.49\left(\mathrm{dd}, J_{\mathrm{H}-\mathrm{H}}=\right.$ $15.6, J_{\mathrm{P}-\mathrm{H}}=9.6 \mathrm{~Hz}, 2 \mathrm{H}, \mathrm{NH}-\mathrm{CH}_{a}-\mathrm{P}$, major diasteromer ), 3.55 $\left(\mathrm{dd}, J_{\mathrm{H}-\mathrm{H}}=15.6 \mathrm{~Hz}, J_{\mathrm{P}-\mathrm{H}}=10.0 \mathrm{~Hz}, \mathrm{NHCH}_{b} \mathrm{P}\right.$, major diasteromer ), $3.56\left(\mathrm{dd}, J_{\mathrm{H}-\mathrm{H}}=15.5 \mathrm{~Hz}, J_{\mathrm{P}-\mathrm{H}}=9.8 \mathrm{~Hz}, \mathrm{NHCH}_{b} \mathrm{P}\right.$, minor diasteromer), $3.99(\mathrm{t}, J=6.6 \mathrm{~Hz}, 1 \mathrm{H}, H \mathrm{C}(\alpha) \mathrm{Leu}$, minor diasteromer), 4.00 (t, $J=6.6 \mathrm{~Hz}, 1 \mathrm{H}, H \mathrm{C}(\alpha) \mathrm{Leu}$, major diasteromer), 4.27 (dd, $J=9.5,5.1 \mathrm{~Hz}, 1 \mathrm{H}, H \mathrm{C}(\alpha) \mathrm{Glu}) ;{ }^{13} \mathrm{C} \mathrm{NMR}(125.77 \mathrm{MHz}$, $\left.\mathrm{D}_{2} \mathrm{O}\right) \delta 13.8\left(\mathrm{~d}, J=92.4 \mathrm{~Hz}, \mathrm{PCH}_{3}\right), 21.5\left(\mathrm{H}_{3} C_{\mathrm{a}}(\delta)\right.$ Leu $), 22.9$ $\left(\mathrm{H}_{3} C_{\mathrm{b}}(\delta) \mathrm{Leu}\right), 25.2(\mathrm{C}(\gamma) \mathrm{Leu}), 26.3(\mathrm{C}(\gamma) \mathrm{Glu}), 31.6(\mathrm{C}(\beta) \mathrm{Glu})$, $\left.40.6(\mathrm{C}(\beta) \mathrm{Leu}), 40.1\left(\mathrm{~d}, J=105.1 \mathrm{~Hz}, \mathrm{NHCH}_{2} \mathrm{P}\right), 40.6 \mathrm{C}(\gamma) \mathrm{Leu}\right)$, $53.3(\mathrm{C}(\alpha)$ Leu $), 53.6(\mathrm{C}(\alpha) \mathrm{Glu}), 172.6(\mathrm{CO}), 175.2(\mathrm{CO}), 175.6$ (CO); ${ }^{31} \mathrm{P}$ NMR $\left(202.45 \mathrm{MHz}, \mathrm{D}_{2} \mathrm{O}\right) \delta 49.1$ (major diasteromer), 48.9 (minor diasteromer). HRMS (ESI), Calcd. for $\mathrm{C}_{13} \mathrm{H}_{27} \mathrm{~N}_{3} \mathrm{O}_{6} \mathrm{P}$ $\left(\mathrm{MH}^{+}\right)$: 352.1637, found: 352.1637 .

Ethyl-[[(L- $\gamma$-glutamyl-L-leucyl)amino]methyl]phosphinate (51). HPLC purification: eluent solvent $\mathrm{H}_{2} \mathrm{O}-\mathrm{MeCN}$-TFA (87:13:0.2), $t_{\mathrm{R}}=10.25 \mathrm{~min}$ : white solid; $99 \%$ yield; ${ }^{1} \mathrm{H} \mathrm{NMR}(500.13 \mathrm{MHz}$, $\left.\mathrm{D}_{2} \mathrm{O}\right) \delta 0.96\left(\mathrm{~d}, J=6.5 \mathrm{~Hz}, 3 \mathrm{H}, H_{3} \mathrm{C}_{\mathrm{a}}(\delta)\right.$ Leu $), 1.01(\mathrm{~d}, J=6.4$ $\left.\mathrm{Hz}, 3 \mathrm{H}, H_{3} \mathrm{C}_{\mathrm{b}}(\delta) \mathrm{Leu}\right), 1.03$ (dt, $J=13.7,7.4 \mathrm{~Hz}, 3 \mathrm{H}, \mathrm{PCH}_{2} \mathrm{CH}_{3}$ ), 1.47-1.71 (m, 5H, $H_{2} \mathrm{C}(\beta)$ Leu, $H \mathrm{C}(\gamma)$ Leu, $\left.\mathrm{PCH}_{2} \mathrm{CH}_{3}\right), 2.08-2.23$ (m, 2H, $\left.H_{2} \mathrm{C}(\beta) \mathrm{Glu}\right), 2.43-2.56\left(\mathrm{~m}, 2 \mathrm{H}, H_{2} \mathrm{C}(\gamma) \mathrm{Glu}\right), 3.49$ (dd, $J_{\mathrm{H}-\mathrm{H}}$ $=15.6 \mathrm{~Hz}, J_{\mathrm{P}-\mathrm{H}}=9.5 \mathrm{~Hz}, 2 \mathrm{H}, \mathrm{NHCH}_{a} \mathrm{P}$, minor diasteromer), 3.50 $\left(\mathrm{dd}, J_{\mathrm{H}-\mathrm{H}}=15.5 \mathrm{~Hz}, J_{\mathrm{P}-\mathrm{H}}=9.5 \mathrm{~Hz}, 2 \mathrm{H}, \mathrm{NHCH}_{a} \mathrm{P}\right.$, major diasteromer ), $3.57\left(\mathrm{dd}, J_{\mathrm{H}-\mathrm{H}}=15.5 \mathrm{~Hz}, J_{\mathrm{P}-\mathrm{H}}=9.5 \mathrm{~Hz}, 1 \mathrm{H}\right.$, $\mathrm{NHCH}_{b} \mathrm{P}$, major diasteromer $), 3.58\left(\mathrm{dd}, J_{\mathrm{H}-\mathrm{H}}=15.6 \mathrm{~Hz}, J_{\mathrm{P}-\mathrm{H}}=\right.$ $9.3 \mathrm{~Hz}, 1 \mathrm{H}, \mathrm{NH}-\mathrm{CH}_{b}-\mathrm{P}$, minor diasteromer ), $3.99(\mathrm{t}, J=6.5 \mathrm{~Hz}$, $1 \mathrm{H}, H \mathrm{C}(\alpha) \mathrm{Leu}$, minor diasteromer), $4.00(\mathrm{t}, J=6.1 \mathrm{~Hz}, 1 \mathrm{H}, H \mathrm{C}-$ $(\alpha)$ Leu $), 4.26(\mathrm{dd}, J=9.2,4.7 \mathrm{~Hz}, 1 \mathrm{H}, H \mathrm{C}(\alpha)$ Glu, minor diasteromer), 4.27 (dd, $J=9.4,5.1 \mathrm{~Hz}, 1 \mathrm{H}, H \mathrm{C}(\alpha) \mathrm{Glu}$, major diasteromer); ${ }^{13} \mathrm{C}$ NMR $\left(125.77 \mathrm{MHz}, \mathrm{D}_{2} \mathrm{O}\right) \delta 7.4(\mathrm{~d}, J=5.1 \mathrm{~Hz}$, $\left.\mathrm{PCH}_{2} \mathrm{CH}_{3}\right), 22.7\left(\mathrm{~d}, J=93.2 \mathrm{~Hz}, \mathrm{PCH}_{2} \mathrm{CH}_{3}\right), 23.2\left(\mathrm{H}_{3} C_{\mathrm{a}}(\delta) \mathrm{Leu}\right)$, $24.7\left(\mathrm{H}_{3} C_{\mathrm{b}}(\delta) \mathrm{Leu}\right), 26.9(\mathrm{C}(\gamma) \mathrm{Leu}), 28.1(\mathrm{C}(\gamma) \mathrm{Glu}), 33.3(\mathrm{C}(\beta)$ Glu), 40.1 (d, $\left.J=98.3 \mathrm{~Hz}, \mathrm{NH}-\mathrm{CH}_{2}-\mathrm{P}\right), 42.4(\mathrm{C}(\beta) \mathrm{Leu}), 55.0$ $(\mathrm{C}(\alpha)$ Leu $), 55.3(\mathrm{C}(\alpha) \mathrm{Glu}), 174.4(\mathrm{CO}), 176.9(\mathrm{CO}), 177.2(\mathrm{CO})$; ${ }^{31} \mathrm{P}$ NMR (202.45 MHz, $\left.\mathrm{D}_{2} \mathrm{O}\right) \delta 50.1$ (major diasteromer), 49.9 (minor diasteromer). HRMS (ESI), Calcd. for $\mathrm{C}_{14} \mathrm{H}_{29} \mathrm{~N}_{3} \mathrm{O}_{6} \mathrm{P}$ $\left(\mathrm{MH}^{+}\right)$: 366.1794, found: 366.1790 .

1-Propyl-[[(L- $\gamma$-glutamyl-L-leucyl)amino]methyl]phosphinate (52). HPLC purification: eluent solvent $\mathrm{H}_{2} \mathrm{O}-\mathrm{MeCN}-\mathrm{TFA}$ (85: 15:0.2), $t_{\mathrm{R}}=10.73$ min: white solid, $99 \%$ yield; ${ }^{1} \mathrm{H}$ NMR $(500.13$ $\left.\mathrm{MHz}, \mathrm{D}_{2} \mathrm{O}\right) \delta 0.97\left(\mathrm{~d}, J=6.1 \mathrm{~Hz}, 3 \mathrm{H}, H_{3} \mathrm{C}_{\mathrm{a}}(\delta)\right.$ Leu $), 1.01(\mathrm{~d}, J=$ $\left.6.2 \mathrm{~Hz}, 3 \mathrm{H}, \mathrm{H}_{3} \mathrm{C}_{\mathrm{b}}(\delta) \mathrm{Leu}\right), 1.06\left(\mathrm{t}, J=7.6 \mathrm{~Hz}, 2 \mathrm{H}, \mathrm{PCH}_{2} \mathrm{CH}_{2} \mathrm{CH}_{3}\right)$, 1.56-1.79 (m, 5H, $\mathrm{H}_{2} \mathrm{C}(\beta)$ Leu, $\mathrm{HC}(\gamma)$ Leu, $\left.\mathrm{P}\left(\mathrm{CH}_{2}\right)_{2} \mathrm{CH}_{3}\right), 2.23-$ $2.36\left(\mathrm{~m}, 2 \mathrm{H}, \mathrm{H}_{2} \mathrm{C}(\beta) \mathrm{Glu}\right), 2.56-2.69\left(\mathrm{~m}, 2 \mathrm{H}, \mathrm{H}_{2} \mathrm{C}(\gamma) \mathrm{Glu}\right), 3.58$ $\left(\mathrm{dd}, J_{\mathrm{H}-\mathrm{H}}=15.6, J_{\mathrm{P}-\mathrm{H}}=9.2 \mathrm{~Hz}, 2 \mathrm{H}, \mathrm{NHCH}_{a} \mathrm{P}\right.$, minor diasteromer), $3.59\left(\mathrm{dd}, J_{\mathrm{H}-\mathrm{H}}=15.7, J_{\mathrm{P}-\mathrm{H}}=9.5 \mathrm{~Hz}, 2 \mathrm{H}, \mathrm{NHCH}_{a} \mathrm{P}\right.$, major diasteromer $), 3.66\left(\mathrm{dd}, J_{\mathrm{H}-\mathrm{H}}=15.7, J_{\mathrm{P}-\mathrm{H}}=9.6 \mathrm{~Hz}, \mathrm{NHCH}_{b} \mathrm{P}\right.$, major diasteromer), $3.67\left(\mathrm{dd}, J_{\mathrm{H}-\mathrm{H}}=15.6, J_{\mathrm{P}-\mathrm{H}}=9.6 \mathrm{~Hz}\right.$, $\mathrm{NHCH}_{b} \mathrm{P}$, minor diasteromer), $4.10(\mathrm{t}, J=6.5 \mathrm{~Hz}, 1 \mathrm{H}, H \mathrm{C}(\alpha)-$ Leu $), 4.26$ (dd, $J=9.6,5.2 \mathrm{~Hz}, 1 \mathrm{H}, H \mathrm{C}(\alpha) \mathrm{Glu}) ;{ }^{13} \mathrm{C}$ NMR $(125.77$ $\left.\mathrm{MHz}, \mathrm{D}_{2} \mathrm{O}\right) \delta 15.8\left(\mathrm{~d}, J=4.2 \mathrm{~Hz}, \mathrm{P}\left(\mathrm{CH}_{2}\right)_{2} \mathrm{CH}_{3}\right), 15.8(\mathrm{~d}, J=16.1$ $\left.\mathrm{Hz}, \mathrm{PCH}_{2} \mathrm{CH}_{2} \mathrm{CH}_{3}\right), 21.5\left(\mathrm{H}_{3} C_{\mathrm{a}}(\delta) \mathrm{Leu}\right), 22.9\left(\mathrm{H}_{3} C_{\mathrm{b}}(\delta) \mathrm{Leu}\right), 25.2$ $(\mathrm{C}(\gamma) \mathrm{Leu}), 26.4(\mathrm{C}(\gamma) \mathrm{Glu}), 30.1\left(\mathrm{~d}, J=93.2 \mathrm{~Hz}, \mathrm{PCH}_{2} \mathrm{CH}_{2} \mathrm{CH}_{3}\right)$, $31.6(\mathrm{C}(\beta) \mathrm{Glu}), 38.9$ (d, $\left.J=97.4 \mathrm{~Hz}, \mathrm{NH}-\mathrm{CH}_{2}-\mathrm{P}\right), 40.6(\mathrm{C}(\beta)-$ Leu), $53.5(\mathrm{C}(\alpha) \mathrm{Leu}), 53.6(\mathrm{C}(\alpha) \mathrm{Glu}), 172.9(\mathrm{CO}), 175.2(\mathrm{CO})$, $175.4(\mathrm{CO}) ;{ }^{31} \mathrm{P}$ NMR $\left(202.45 \mathrm{MHz}, \mathrm{D}_{2} \mathrm{O}\right) \delta 49.7$ (major diasteromer), 49.5 (minor diasteromer). HRMS (ESI), Calcd. for $\mathrm{C}_{15} \mathrm{H}_{31} \mathrm{~N}_{3} \mathrm{O}_{6} \mathrm{P}\left(\mathrm{MH}^{+}\right)$: 380.1950 , found: 380.1945 .

1-Butyl-[[(L- $\gamma$-glutamyl-L-leucyl)amino]methyl]phosphinate (53). HPLC purification: eluent solvent $\mathrm{H}_{2} \mathrm{O}-\mathrm{MeCN}$-TFA (80:20: $0.2), t_{\mathrm{R}}=9.14 \mathrm{~min}:$ white solid; ${ }^{1} \mathrm{H}$ NMR $\left(500.13 \mathrm{MHz}, \mathrm{CD}_{3} \mathrm{OD}\right)$ $\delta 0.91\left(\mathrm{~d}, J=6.5 \mathrm{~Hz}, 3 \mathrm{H}, H_{3} \mathrm{C}_{\mathrm{a}}(\delta) \mathrm{Leu}\right), 0.93(\mathrm{t}, J=6.6 \mathrm{~Hz}, 3 \mathrm{H}$, $\left.\mathrm{P}\left(\mathrm{CH}_{2}\right)_{3} \mathrm{CH}_{3}\right), 0.96\left(\mathrm{~d}, J=6.5 \mathrm{~Hz}, 3 \mathrm{H}, \mathrm{H}_{3} \mathrm{C}_{\mathrm{b}}(\delta) \mathrm{Leu}\right), 1.36-1.44$ $\left(\mathrm{m}, 4 \mathrm{H}, \mathrm{P}\left(\mathrm{CH}_{2}\right)_{2} \mathrm{CH}_{2} \mathrm{CH}_{3}\right), 1.50-1.72\left(\mathrm{~m}, 7 \mathrm{H}, \mathrm{P}\left(\mathrm{CH}_{2}\right)_{2} \mathrm{CH}_{2} \mathrm{CH}_{3}\right.$, $\left.\mathrm{H}_{2} \mathrm{C}(\beta) \mathrm{Leu}, H \mathrm{C}(\gamma) \mathrm{Leu}\right), 2.14-2.21\left(\mathrm{~m}, 2 \mathrm{H}, H_{2} \mathrm{C}(\beta) \mathrm{Glu}\right), 2.48-$ $2.59\left(\mathrm{~m}, 2 \mathrm{H}, \mathrm{H}_{2} \mathrm{C}(\gamma) \mathrm{Glu}\right), 3.36\left(\mathrm{dd}, J_{\mathrm{H}-\mathrm{H}}=14.8 \mathrm{~Hz}, J_{\mathrm{P}-\mathrm{H}}=10.1\right.$ $\left.\mathrm{Hz}, 2 \mathrm{H}, \mathrm{NH}-\mathrm{CH}_{a}-\mathrm{P}\right), 3.46\left(\mathrm{dd}, J_{\mathrm{H}-\mathrm{H}}=14.8 \mathrm{~Hz}, J_{\mathrm{P}-\mathrm{H}}=7.9 \mathrm{~Hz}\right.$, $\left.\mathrm{NHCH}_{b} \mathrm{P}\right), 3.89(\mathrm{t}, J=6.0 \mathrm{~Hz}, 1 \mathrm{H}, H \mathrm{C}(\alpha)$ Leu $), 4.27$ (t, $J=7.3$ $\mathrm{Hz}, 1 \mathrm{H}, H \mathrm{C}(\alpha) \mathrm{Glu}) ;{ }^{13} \mathrm{C}$ NMR $\left(125.77 \mathrm{MHz}, \mathrm{CD}_{3} \mathrm{OD}\right) \delta 14.0$ $\left(\mathrm{P}\left(\mathrm{CH}_{2}\right)_{3} \mathrm{CH}_{3}\right), 21.8 \quad\left(\mathrm{H}_{3} C_{\mathrm{a}}(\delta) \mathrm{Leu}\right), 23.4 \quad\left(\mathrm{H}_{3} C_{\mathrm{b}}(\delta) \mathrm{Leu}\right), 24.9$ $\left(\mathrm{P}\left(\mathrm{CH}_{2}\right)_{2} \mathrm{CH}_{2} \mathrm{CH}_{3}\right), 25.1\left(\mathrm{~d}, J=16.7 \mathrm{~Hz}, \mathrm{P}\left(\mathrm{CH}_{2} \mathrm{CH}_{2} \mathrm{CH}_{2} \mathrm{CH}_{3}\right), 25.9\right.$ $(\mathrm{C}(\gamma) \mathrm{Leu}), 27.0(\mathrm{C}(\gamma) \mathrm{Glu}), 32.3(\mathrm{C}(\beta) \mathrm{Glu}), 41.6(\mathrm{C}(\beta) \mathrm{Leu}), 53.6$ $(\mathrm{C}(\alpha)$ Leu $), 174.5$ (CO), 174.7 (CO); ${ }^{31} \mathrm{P}$ NMR $\left(202.45 \mathrm{MHz}, \mathrm{CD}_{3^{-}}\right.$ OD) $\delta$ 45.0.; HRMS (ESI), Calcd. for $\mathrm{C}_{16} \mathrm{H}_{33} \mathrm{~N}_{3} \mathrm{O}_{6} \mathrm{P}\left(\mathrm{MH}^{+}\right)$: 394.2107, found: 394.2124 .

1-Pentyl-[[(L- $\gamma$-glutamyl-L-leucyl $)$ amino]methyl]phosphinate (54). HPLC purification: eluent solvent $\mathrm{H}_{2} \mathrm{O}-\mathrm{MeCN}-\mathrm{TFA}$ (75: 25:0.2), $t_{\mathrm{R}}=10.39 \mathrm{~min}:$ white solid; $100 \%$ yield; ${ }^{1} \mathrm{H}$ NMR $(500.13$ $\left.\mathrm{MHz}, \mathrm{CD}_{3} \mathrm{OD}\right) \delta 0.89\left(\mathrm{t}, J=6.6 \mathrm{~Hz}, 3 \mathrm{H}, \mathrm{P}\left(\mathrm{CH}_{2}\right)_{4} \mathrm{CH}_{3}\right), 0.90(\mathrm{~d}$, $\left.J=6.6 \mathrm{~Hz}, 3 \mathrm{H}, H_{3} \mathrm{C}_{\mathrm{a}}(\delta) \mathrm{Leu}\right), 0.94\left(\mathrm{~d}, J=6.6 \mathrm{~Hz}, 3 \mathrm{H}, H_{3} \mathrm{C}_{\mathrm{b}}(\delta)-\right.$ Leu), $1.30-1.41\left(\mathrm{~m}, 4 \mathrm{H}, \mathrm{P}\left(\mathrm{CH}_{2}\right)_{2}\left(\mathrm{CH}_{2}\right)_{2} \mathrm{CH}_{3}\right) 1.54-1.63(\mathrm{~m}, 4 \mathrm{H}$, $\left.\mathrm{P}\left(\mathrm{CH}_{2}\right)_{2}\left(\mathrm{CH}_{2}\right)_{2} \mathrm{CH}_{3}\right)$ 1.63-1.73 (m, 3H, $\mathrm{H}_{2} \mathrm{C}(\beta)$ Leu, $H \mathrm{C}(\gamma)$ Leu $)$, 2.07-2.28 (m, 2H, $\left.H_{2} \mathrm{C}(\beta) \mathrm{Glu}\right), 2.48-2.59\left(\mathrm{~m}, 2 \mathrm{H}, H_{2} \mathrm{C}(\gamma) \mathrm{Glu}\right)$, $3.39\left(\mathrm{dd}, J_{\mathrm{H}-\mathrm{H}}=15.5 \mathrm{~Hz}, J_{\mathrm{P}-\mathrm{H}}=9.8 \mathrm{~Hz}, 2 \mathrm{H}, \mathrm{NH}-\mathrm{CH}_{a}-\mathrm{P}\right), 3.53$ $\left(\mathrm{dd}, J_{\mathrm{H}-\mathrm{H}}=15.5 \mathrm{~Hz}, J_{\mathrm{P}-\mathrm{H}}=8.9 \mathrm{~Hz}, \mathrm{NHCH}_{b} \mathrm{P}\right), 4.00(\mathrm{t}, J=6.4$ $\mathrm{Hz}, 1 \mathrm{H}, H \mathrm{C}(\alpha) \mathrm{Leu}), 4.30(\mathrm{t}, J=7.5 \mathrm{~Hz}, 1 \mathrm{H}, H \mathrm{C}(\alpha) \mathrm{Glu}) ;{ }^{13} \mathrm{C} \mathrm{NMR}$

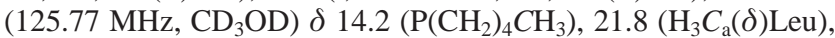
$22.2\left(\mathrm{~d}, J=4.2 \mathrm{~Hz}, \mathrm{PCH}_{2} \mathrm{CH}_{2}\left(\mathrm{CH}_{2}\right)_{2} \mathrm{CH}_{3}\right), 23.2\left(\mathrm{P}\left(\mathrm{CH}_{2}\right)_{3} \mathrm{CH}_{2} \mathrm{CH}_{3}\right)$, $23.4\left(\mathrm{H}_{3} C_{\mathrm{b}}(\delta) \mathrm{Leu}\right), 25.9(\mathrm{C}(\gamma) \mathrm{Leu}), 27.0(\mathrm{C}(\gamma) \mathrm{Glu}), 28.5(\mathrm{~d}, J=$ $\left.91.6 \mathrm{~Hz}, \mathrm{PCH}_{2}\left(\mathrm{CH}_{2}\right)_{3} \mathrm{CH}_{3}\right), 32.2(\mathrm{C}(\beta) \mathrm{Glu}), 34.2(\mathrm{~d}, J=16.1 \mathrm{~Hz}$, $\left.\mathrm{P}\left(\mathrm{CH}_{2}\right)_{2} \mathrm{CH}_{2} \mathrm{CH}_{2} \mathrm{CH}_{3}\right), 40.0\left(\mathrm{~d}, J=100.9 \mathrm{~Hz}, \mathrm{NHCH}_{2} \mathrm{P}\right), 41.7$ 
$(\mathrm{C}(\beta)$ Leu $), 53.5(\mathrm{C}(\alpha)$ Leu $), 55.6(\mathrm{C}(\alpha) \mathrm{Glu}), 171.5(\mathrm{CO}), 174.5$ (CO), 174.8 (CO); ${ }^{31} \mathrm{P}$ NMR (202.45 MHz, $\left.\mathrm{CD}_{3} \mathrm{OD}\right) \delta 47.3$; HRMS (ESI), Calcd. for $\mathrm{C}_{17} \mathrm{H}_{35} \mathrm{~N}_{3} \mathrm{O}_{6} \mathrm{P}\left(\mathrm{MH}^{+}\right)$: 402.2263 , found: 402.2278 .

1-Hexyl-[[( $L$ - $\gamma$-glutamyl-L-leucyl $)$ amino $]$ methyl $]$ phosphinate (55). HPLC purification: eluent solvent $\mathrm{H}_{2} \mathrm{O}-\mathrm{MeCN}-\mathrm{TFA}$ (75: 25:0.2), $t_{\mathrm{R}}=17.38 \mathrm{~min}:$ white solid; $98 \%$ yield; ${ }^{1} \mathrm{H}$ NMR $(500.13$ $\left.\mathrm{MHz}, \mathrm{CD}_{3} \mathrm{OD}\right) \delta 0.90\left(\mathrm{t}, J=7.0 \mathrm{~Hz}, 3 \mathrm{H}, \mathrm{P}\left(\mathrm{CH}_{2}\right)_{5} \mathrm{CH}_{3}\right), 0.92$ (d, $\left.J=6.4 \mathrm{~Hz}, 3 \mathrm{H}, H_{3} \mathrm{C}_{\mathrm{a}}(\delta) \mathrm{Leu}\right), 0.96\left(\mathrm{~d}, J=6.4 \mathrm{~Hz}, 3 \mathrm{H}, H_{3} \mathrm{C}_{\mathrm{b}}(\delta)-\right.$ Leu ), 1.26-1.41 (m, 6H, $\left.\mathrm{P}\left(\mathrm{CH}_{2}\right)_{2}\left(\mathrm{CH}_{2}\right)_{3} \mathrm{CH}_{3}\right), 1.54-1.62(\mathrm{~m}, 4 \mathrm{H}$, $\left.\mathrm{P}\left(\mathrm{CH}_{2}\right)_{2}\left(\mathrm{CH}_{2}\right)_{2} \mathrm{CH}_{3}\right), 1.63-1.72\left(\mathrm{~m}, 3 \mathrm{H}, H_{2} \mathrm{C}(\beta)\right.$ Leu, $H \mathrm{C}(\gamma)$ Leu $)$, $2.08-2.29\left(\mathrm{~m}, 2 \mathrm{H}, H_{2} \mathrm{C}(\beta) \mathrm{Glu}\right), 2.48 .-2.59\left(\mathrm{~m}, 2 \mathrm{H}, H_{2} \mathrm{C}(\gamma) \mathrm{Glu}\right)$, $3.46\left(\mathrm{dd}, J_{\mathrm{H}-\mathrm{H}}=15.4 \mathrm{~Hz}, J_{\mathrm{P}-\mathrm{H}}=8.8 \mathrm{~Hz}, 2 \mathrm{H}, \mathrm{NH}-\mathrm{CH}_{a}-\mathrm{P}\right), 3.61$ $\left(\mathrm{dd}, J_{\mathrm{H}-\mathrm{H}}=15.4 \mathrm{~Hz}, J_{\mathrm{P}-\mathrm{H}}=8.7 \mathrm{~Hz}, \mathrm{NHCH}_{b} \mathrm{P}\right), 4.00(\mathrm{t}, J=6.3$ $\mathrm{Hz}, 1 \mathrm{H}, H \mathrm{C}(\alpha) \mathrm{Leu}), 4.36(\mathrm{t}, J=7.5 \mathrm{~Hz}, 1 \mathrm{H}, H \mathrm{C}(\alpha) \mathrm{Glu}) ; \delta 14.4$ $\left(\mathrm{P}\left(\mathrm{CH}_{2}\right)_{5} \mathrm{CH}_{3}\right), 21.8\left(\mathrm{H}_{3} \mathrm{C}_{\mathrm{a}}(\delta) \mathrm{Leu}\right), 22.7\left(\mathrm{PCH}_{2} \mathrm{CH}_{2}\left(\mathrm{CH}_{2}\right)_{3} \mathrm{CH}_{3}\right), 23.4$ $\left(\mathrm{H}_{3} C_{\mathrm{b}}(\delta) \mathrm{Leu}\right), 23.5\left(\mathrm{P}\left(\mathrm{CH}_{2}\right)_{4} \mathrm{CH}_{2} \mathrm{CH}_{3}\right), 26.0(\mathrm{C}(\gamma) \mathrm{Leu}), 27.0(\mathrm{C}(\gamma)-$ Glu), 31.3 (d, $\left.J=89.9 \mathrm{~Hz}, \mathrm{PCH}_{2}\left(\mathrm{CH}_{2}\right)_{4} \mathrm{CH}_{3}\right), 34.3$ (d, $J=14.4$ $\left.\mathrm{Hz}, \mathrm{P}\left(\mathrm{CH}_{2}\right)_{2} \mathrm{CH}_{2}\left(\mathrm{CH}_{2}\right)_{2} \mathrm{CH}_{3}\right), 32.2(\mathrm{C}(\beta) \mathrm{Glu}), 32.5\left(\mathrm{P}\left(\mathrm{CH}_{2}\right)_{3} \mathrm{CH}_{2}-\right.$ $\left.\mathrm{CH}_{2} \mathrm{CH}_{3}\right), 41.6\left(\mathrm{~d}, J=100.9 \mathrm{~Hz}, \mathrm{NHCH}_{2} \mathrm{P}\right), 41.7(\mathrm{C}(\beta) \mathrm{Leu}), 53.5$ $(\mathrm{C}(\alpha) \mathrm{Leu}), 55.6(\mathrm{C}(\alpha) \mathrm{Glu}), 171.6(\mathrm{CO}), 174.5(\mathrm{CO}), 174.8(\mathrm{CO})$; ${ }^{31} \mathrm{P}$ NMR (202.45 MHz, $\left.\mathrm{CD}_{3} \mathrm{OD}\right) \delta 46.4$; HRMS (ESI), Calcd. for $\mathrm{C}_{18} \mathrm{H}_{37} \mathrm{~N}_{3} \mathrm{O}_{6} \mathrm{P}\left(\mathrm{MH}^{+}\right)$: 422.2420, found: 422.2421 .

1-Heptyl-[[(L- $\gamma$-glutamyl-L-leucyl)amino]methyl]phosphinate (56). HPLC purification: eluent solvent $\mathrm{H}_{2} \mathrm{O}-\mathrm{MeCN}-\mathrm{TFA}$ (70: 30:0.2), $t_{\mathrm{R}}=13.96 \mathrm{~min}:$ white solid; $99 \%$ yield; ${ }^{1} \mathrm{H}$ NMR $(500$ $\left.\mathrm{MHz}, \mathrm{CD}_{3} \mathrm{OD}\right) \delta 0.89\left(\mathrm{t}, J=7.0 \mathrm{~Hz}, 3 \mathrm{H}, \mathrm{P}\left(\mathrm{CH}_{2}\right)_{6} \mathrm{CH}_{3}\right), 0.91(\mathrm{~d}$, $\left.J=6.2 \mathrm{~Hz}, 3 \mathrm{H}, H_{3} \mathrm{C}_{\mathrm{a}}(\delta) \mathrm{Leu}\right), 0.95\left(\mathrm{~d}, J=6.2 \mathrm{~Hz}, 3 \mathrm{H}, H_{3} \mathrm{C}_{\mathrm{b}}(\delta)-\right.$ Leu ), $1.23-1.42\left(\mathrm{~m}, 4 \mathrm{H}, \mathrm{P}\left(\mathrm{CH}_{2}\right)_{2}\left(\mathrm{CH}_{2}\right)_{4} \mathrm{CH}_{3}\right), 1.45-1.75(\mathrm{~m}, 7 \mathrm{H}$, $\mathrm{H}_{2} \mathrm{C}(\beta)$ Leu, $\mathrm{HC}(\gamma)$ Leu, $\left.\mathrm{P}\left(\mathrm{CH}_{2}\right)_{2}\left(\mathrm{CH}_{2}\right)_{4} \mathrm{CH}_{3}\right), 2.07-2.28(\mathrm{~m}, 2 \mathrm{H}$, $\left.\mathrm{H}_{2} \mathrm{C}(\beta) \mathrm{Glu}\right), 2.44 .-2.63\left(\mathrm{~m}, 2 \mathrm{H}, \mathrm{H}_{2} \mathrm{C}(\gamma) \mathrm{Glu}\right), 3.26\left(\mathrm{dd}, J_{\mathrm{H}-\mathrm{H}}=14.7\right.$ $\left.\mathrm{Hz}, J_{\mathrm{P}-\mathrm{H}}=9.8 \mathrm{~Hz}, 2 \mathrm{H}, \mathrm{NH}-\mathrm{CH}_{a}-\mathrm{P}\right), 3.38\left(\mathrm{dd}, J_{\mathrm{H}-\mathrm{H}}=114.7 \mathrm{~Hz}\right.$, $\left.J_{\mathrm{P}-\mathrm{H}}=8.8 \mathrm{~Hz}, \mathrm{NHCH}_{b} \mathrm{P}\right), 3.89(\mathrm{t}, J=6.0 \mathrm{~Hz}, 1 \mathrm{H}, H \mathrm{C}(\alpha) \mathrm{Leu})$, $4.36(\mathrm{dd}, J=10.1,4.4 \mathrm{~Hz}, 1 \mathrm{H}, H \mathrm{C}(\alpha) \mathrm{Glu}) ;{ }^{13} \mathrm{C}$ NMR $(125.77$ $\left.\mathrm{MHz}, \mathrm{CD}_{3} \mathrm{OD}\right) \delta 14.4\left(\mathrm{P}\left(\mathrm{CH}_{2}\right)_{6} C_{3}\right), 21.8\left(\mathrm{H}_{3} C_{\mathrm{a}}(\delta) \mathrm{Leu}\right), 22.8$

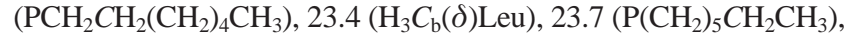
$26.0(\mathrm{C}(\gamma) \mathrm{Leu}), 27.0(\mathrm{C}(\gamma) \mathrm{Glu}), 28.9\left(\mathrm{~d}, J=87.3 \mathrm{~Hz}, \mathrm{PCH}_{2}\left(\mathrm{CH}_{2}\right)_{5^{-}}\right.$ $\left.\mathrm{CH}_{3}\right), 30.0\left(\mathrm{P}\left(\mathrm{CH}_{2}\right)_{3} \mathrm{CH}_{2}(\mathrm{CH} 2)_{2} \mathrm{CH}_{3}\right), 32.1(\mathrm{~d}, J=15.3 \mathrm{~Hz}$, $\left.\mathrm{P}\left(\mathrm{CH}_{2}\right)_{2} \mathrm{CH}_{2}\left(\mathrm{CH}_{2}\right)_{3} \mathrm{CH}_{3}\right), 32.3(\mathrm{C}(\beta) \mathrm{Glu}), 32.8\left(\mathrm{P}\left(\mathrm{CH}_{2}\right)_{4} \mathrm{CH}_{2} \mathrm{CH}_{2}-\right.$ $\left.\mathrm{CH}_{3}\right), 39.2\left(\mathrm{~d}, J=101.7 \mathrm{~Hz}, \mathrm{NHCH}_{2} \mathrm{P}\right), 41.7(\mathrm{C}(\beta) \mathrm{Leu}), 53.7$ $(\mathrm{C}(\alpha) \mathrm{Leu}), 54.4(\mathrm{C}(\alpha) \mathrm{Glu}), 172.5(\mathrm{CO}), 174.4(\mathrm{CO}), 174.8(\mathrm{CO})$; ${ }^{31} \mathrm{P}$ NMR $\left(202.45 \mathrm{MHz}, \mathrm{CD}_{3} \mathrm{OD}\right) \delta 45.0$; HRMS (ESI), Calcd. for $\mathrm{C}_{19} \mathrm{H}_{39} \mathrm{~N}_{3} \mathrm{O}_{6} \mathrm{P}\left(\mathrm{MH}^{+}\right)$: 436.2576, found: 436.2584 .

Uracil Incorporation in Amastigotes. 1. Inhibition of Replication. Gamma-irradiated $\mathrm{L}_{6} \mathrm{E}_{9}$ myoblasts $\left(1 \times 10^{7}\right.$ cells/plate $)$ in DMEM medium containing $20 \%$ fetal calf serum were plated in 12-well tissue culture plates and incubated at $37{ }^{\circ} \mathrm{C}$ in a $27 \% \mathrm{CO}_{2}$ atmosphere for $24 \mathrm{~h}$. After $24 \mathrm{~h}$, wells were washed once, and fresh media was added containing $4.17 \times 10^{6}$ trypomastigotes/well. One well was left without parasites for control. After $2 \mathrm{~h}$ incubation at $37{ }^{\circ} \mathrm{C}$ in a $7 \% \mathrm{CO}_{2}$ atmosphere, cultures were washed twice with Hank's solution, and culture medium containing $1 \mu \mathrm{Ci}$ of $\left[5,6-{ }^{3} \mathrm{H}\right]-$ uracil $/ \mathrm{mL}$ (specific activity, 40-50 Ci/mmol; NEN Research Products, Boston) was added, $1 \mathrm{~mL} / \mathrm{well}$. At this time, drugs solutions in absolute ethanol $(10 \mu \mathrm{l})$ were added. Two wells were left for infection control. $10 \mu \mathrm{L}$ of absolute ethanol was added to control and infection control wells. Incorporation of the $\left[{ }^{3} \mathrm{H}\right]$ into trichloroacetic acid (TCA)-precipitable material was measured at day 3 . The supernatants of the monolayers were transferred to glass tubes. The cells were dissolved with $1.3 \mathrm{~mL}$ of $1 \%$ sodium dodecyl sulfate containing $100 \mu \mathrm{L}$ of cold uracil per $\mathrm{mL}$, and the suspension was transferred to the glass tubes. The wells were rinsed with 3 $\mathrm{mL}$ of $0.3 \mathrm{~N}$ TCA (5\%) (ice-cold) which was combined with the previous suspension. The samples were maintained in ice for 15 min and collected on glass fiber filters (Whatman GF/D) by using a sampling manifold (Millipore, Bedford, MA), the tubes were rinsed three times with $4 \mathrm{~mL}$ of $0.3 \mathrm{~N}$ TCA, as were the filters, twice with TCA, and once with $95 \%$ ethanol. After drying the filters, they were placed in $5 \mathrm{~mL}$ of scintillation cocktail Econolume. Vials with the filters and cocktails were vortexed vigorously and counted.

2. Inhibition of Invasion. The same protocol was carried out, but drug solutions were added at the invasion time.
Drug Testing against Epimastigotes. Trypanosoma cruzi epimastigotes (Y strain) were grown in LIT $+5 \% \mathrm{NCS}+1 \% \mathrm{P} / \mathrm{S}$ medium. Five day old culture was centrifuged and resuspended in fresh medium to get a $2-3 \times 10^{6} \mathrm{cell} / \mathrm{mL}$ suspension (as determined by counting in a Neubauer chamber). Parasites were then placed in sterile screw-cap tubes $(2 \mathrm{~mL} /$ tube). Two tubes were filled with medium for blank. Each drug was tested at four different concentrations $(1,5,15$, and $25 \mu \mathrm{g} / \mathrm{mL})$, each one in triplicate. Drugs were dissolved in absolute ethanol. A control without drug was done for each group tested. An equivalent amount of ethanol $(20 \mu \mathrm{L})$ was added to blank and control tubes. The concentration of cells was determined by measuring the absorbance of the culture medium containing parasites at $600 \mathrm{~nm}$ against a blank with culture medium alone. To calculate percent inhibition, the following formula was used: Percent inhibition $=100-\left(\Delta A_{\mathrm{d}} \times 100\right) / \Delta A_{\mathrm{c}}$, where $\Delta A_{\mathrm{c}}$ and $\Delta A_{\mathrm{d}}$ are the differences in the absorbance of control cultures and drug-treated cultures, respectively, at the beginning and at the end of the experiment. The maximum amount of solvent used (1\% ethanol) did not have any significant effect on the epimastigotes growth. $\mathbf{5 7}$ was used as a positive control.

Acknowledgment. This work was supported by grants from the European Commission INCO-DC, Fundación Antorchas, the National Research Council of Argentina (PIP 635/98), and the Universidad de Buenos Aires (X-252) to J.B.R., and the U.S. National Institutes of Health (GM-65307) to R.D.

Supporting Information Available: ${ }^{1} \mathrm{H},{ }^{13} \mathrm{C}$, and ${ }^{31} \mathrm{P}$ NMR spectra, copies of the HRMS data, and HPLC analyses for the title compounds. This material is available free of charge via the Internet at http://pubs.acs.org.

\section{References}

(1) Fairlamb, A. H.; Blackburn, P.; Ulrich, P.; Chait, B. T.; Cerami, A Trypanothione: a novel bis(glutathionyl)spermidine cofactor for glutathione reductase in trypanosomatids Science 1985, 227, 14851487

(2) Flohé, L.; Hecht, J.; Steinert, P. Glutathione and trypanothione in parasitic hydroperoxide metabolism. Free Radic. Biol. Med. 1999, 27, 966-984.

(3) Augustyns, K.; Amssoms, A.; Yamani, A.; Rajan, P. K.; Haemers, A. Trypanothione as a target in the design of antitrypanosomal and antileishmanial agents. Curr. Pharm. Des. 2001, 7, 1117-1141.

(4) Steinert, P.; Dittmar, K.; Kalisz, H. M.; Montemartini, M.; Nogoceke, E.; Rodhe, M.; Singh, M.; Flohé, L. Cytoplasmic localization of the trypanothione peroxidase system in Crithidia fasciculata. Free Radic. Biol. Med. 1999, 26, 844-849.

(5) Henderson, G. B.; Glushka, J.; Cowburn, D.; Cerami, A. Synthesis and NMR characterization of the trypanosomatid metabolite, $N^{1}, N^{8}$ bis(glutathionyl)spermidine disulphide (trypanothione disulphide). $J$. Chem. Soc., Perkin Trans. 1 1990, 911-914.

(6) Fairlamb, A. H.; Cerami, A. Identification of a novel, thiol-containing cofactor essential for glutathione reductase enzyme activity in trypanosomatids. Mol. Biochem. Parasitol. 1985, 14, 187-198.

(7) Shames, S. L.; Fairlamb, A. H.; Cerami, A.; Walsh, C. T. Purification and characterization of trypanothione reductase from Crithidia fasciculata, a newly discovered member of the family of disulfidecontaining flavoprotein reductases. Biochemistry 1986, 25, 35193526.

(8) Fairlamb, A. H. Metabolism and functions of trypanothione in the Kinetoplastida. Annu. Rev. Microbiol. 1992, 46, 695-729.

(9) Montemartini, M.; Kalisz, H. M.; Hecht, H.-J.; Steinert, P., Flohé, L. Activation of active-site cysteine residues in the peroxiredoxintype tryparedoxin peroxidase of Crithidia fasciculata. Eur. J. Biochem. 1999, 264, 516-524.

(10) Wilkinson, S. R.; Temperton, N. J.; Mondragon, A.; Kelly, J. M. Distinct mitochondrial and cytosolic enzymes mediate trypanothionedependent peroxide metabolism in Trypanosoma cruzi. J. Biol. Chem. 2000, 175, 8220-8225.

(11) Vickers T. J.; Wyllie, S.; Fairlamb, A. H. Leishmania major elongation factor 1B complex has trypanothione $S$-transferase and peroxidase activity. J. Biol. Chem. 2004, 279, 49003-49009.

(12) Vickers T. J.; Fairlamb A. H. Trypanothione $S$-transferase activity in a trypanosomatid ribosomal elongation factor 1B. J. Biol. Chem. 2004, 279, 27246-27256. 
(13) Lueder, D. V.; Phillips, M. A. Characterization of Trypanosoma brucei $\gamma$-glutamylcysteine synthetase, an essential enzyme in the biosynthesis of trypanothione (diglutathionylspermidine). J. Biol. Chem. 1996, 271, 17485-17490.

(14) Drew, R.; Miners, J. O. The effects of buthionine sulphoximine (BSO) on glutathione depletion and xenobiotic biotransformation Biochem. Pharmacol. 1984, 33, 2989-2994.

(15) González, N. S.; Huber, A.; Algranati, I. D. Spermidine is essential for normal proliferation of trypanosomatid protozoa. FEBS Lett. 2001, 508, 323-326

(16) Kierszembaum, F.; Wirth, J. J.; McCann, P. P.; Sjoerdsma. Arginine decarboxylase inhibitors reduce the capacity of Trypanosoma cruzi to infect and multiply in mammalian host cells A. Proc. Natl. Acad. Sci. U.S.A. 1987, 84, 4278-4282.

(17) Ariyanayagam, M. R.; Fairlamb, A. H. Diamine auxotrophy may be a universal feature of Trypanosoma cruzi epimastigotes. Mol. Biochem. Parasitol. 1997, 84, 111-121.

(18) Hernández, S.; Schwarcz De Tarlovsky, M. Arginine decarboxylase in Trypanosoma cruzi, characteristics and kinetic properties Cell. Mol. Biol. 1999, 45, 383-391.

(19) Pegg, A. E.; McCann, P. P. Polyamine metabolism and function in mammalian-cells and protozoans; ISI Atlas of Science 1988, 11-18.

(20) Basselin, M.; Coombs, G. H.; Barret, M. P. Putrescine and spermidine transport in Leishmania. Mol. Biochem. Parasitol. 2000, 109, 3746.

(21) Henderson, G. B.; Yamaguchi, M.; Novoa, L.; Fairlamb, A. H. Cerami, A. Biosynthesis of the trypanosomatid metabolite trypanothione: purification and characterization of trypanothione synthetase from Crithidia fasciculata. Biochemistry 1990, 29, 3924-3929.

(22) Smith, K.; Nadeau, K.; Bradley M.; Walsh, C.; Fairlamb, A. H Purification of glutathionylspermidine and trypanothione synthetases from Crithidia fasciculata. Prot. Sci. 1992, 1, 874-883.

(23) Koening, K.; Menge, U.; Kiess, M.; Wray, V.; Flohé, L. Convenient isolation and kinetic mechanism of glutathionylspermidine synthetase from Crithidia fasciculata. J. Biol. Chem. 1997, 18, 11908-11915.

(24) Bollinger, J. M.; Kwon, D. S.; Huisman, G. W.; Kolters, R.; Walsh, C. T. Glutathionylspermidine metabolism in Escherichia coli. Purification, cloning, overproduction, and characterization of a bifunctional glutathionylspermidine synthetase/amidase. J. Biol. Chem. 1995, 23, 14031-14041.

(25) Tetaud, E.; Fairlamb, A. H. Cloning, expression and reconstitution of the trypanothione-dependent peroxidase system of Crithidia fasciculata. Mol Biochem Parasitol. 1998, 96, 111-123.

(26) Oza, S. L.; Ariyanayagam, M. R.; Fairlamb, A. H. Characterization of recombinant glutathionylspermidine synthetase/amidase from Crithidia fasciculata. Biochem. J. 2002, 364, 679-686.

(27) Oza, S. L.; Tetaud, E.; Ariyanayagam, M. R.; Warnon, S. S.; Fairlamb, A. H. A single enzyme catalyses formation of trypanothione from glutathione and spermidine in Trypanosoma cruzi. J. Biol. Chem. 2002, 39, 35853-35861.

(28) Oza, S. L.; Ariyanayagam, M. R.; Aitcheson, N.; Fairlamb, A. H Properties of trypanothione synthethase from Trypanosoma brucei. Mol. Biochem. Parasitol. 2003, 131, 25-33.

(29) Comini, M.; Menge, U.; Wissing, J.; Flohé, L. Trypanothione synthesis in Crithidia Revisited. J. Biol. Chem. 2005, 280, 68506860 .

(30) Oza, S. L.; Shaw, M. P.; Wyllie, S.; Fairlamb, A. H. Trypanothione biosynthesis in Leishmania major. Mol. Biochem. Parasitol. 2005 139, 107-116.

(31) Ariyanayagam, M. R.; Oza, S. L.; Mehlert, A.; Fairlamb, A. H. Bis(glutathionylspermidine) and other novel trypanothione analogues in Trypanosoma cruzi. J. Biol. Chem. 2003, 278, 27612-2769.

(32) Jacoby, E. M.; Schlichting, I.; Lantwin, C. B.; Kabsch, W.; KrauthSiegel, R. L. Proteins 1996, 24, 73-80.

(33) Zhang, Y.; Bond, C. S.; Bailey, S.; Cunningham, M. L.; Fairlamb A. H.; Hunter, W. N. Protein Sci. 1996, 5, 52-61.

(34) Bailey, S.; Smith, K.; Fairlamb, A. H.; Hunter, W. N. Substrate interactions between trypanothione reductase and $N^{1}$-glutathionylspermidine disulphide at $0.28-\mathrm{nm}$ resolution. Eur. J. Biochem. 1993, 213, 67-75.

(35) De Craecker, S.; Verbruggen, C.; Rajan, P. K.; Smith, K.; Haemers, A.; Fairlamb, A. H. Characterization of the peptide substrate specificity of glutathionylspermidine synthetase from Crithidia fasciculata. Mol. Biochem. Parasitol. 1997, 84, 25-32.

(36) Fan, C.; Moews, P. C.; Walsh, C. T.; Knox, J. R. Vancomycin resistance: structure of D-alanine: D-alanine ligase at $2.3 \AA$ resolution. Science 1994, 266, 439-443.

(37) Hiratake, J.; Kato, H.; Oda, J. Mechanism-Based Inactivation of Glutathione Synthetase by Phosphinic Acid Transition-State Analogue. J. Am. Chem. Soc. 1994, 116, 12059-12060.
(38) Verbruggen, C.; De Craecker, S.; Rajan, P. K.; Jiago, X.-Y.; Borloo, M.; Smith, K.; Fairlamb, A. H.; Haemers, A. Phosphonic acid and phosphinic acid tripeptides as iniiibitors of glutatltlonylspermidine synthetase. Bioorg. Med. Chem. Lett. 1996, 6, 253-258.

(39) Chen, S.; Coward, J. K. Investigations on New Strategies for the Facile Synthesis of Polyfunctionalized Phosphinates: Phosphinopeptide Analogues of Glutathionylspermidine. J. Org. Chem. 1998, 63, 502-509.

(40) Chen, S.; Lin, C.-H.; Walsh, C. T.; Coward, J. K. Novel inhibitors of trypanothione biosynthesis: synthesis and evaluation of a phosphinate analogue of glutathionyl spermidine (GSP), a potent, slowbinding inhibitor of GSP synthetase. Bioorg. Med. Chem. Lett. 1997, 7, 505-510.

(41) Amssoms, K.; Oza, S. L.; Ravaschino, E.; Yamani, A.; Lambeir, A.; Rajan, P.; Bal, G.; Rodriguez, J. B.; Fairlamb, A. H.; Augustyns, K.; Haemers, A. Glutathione-like Tripeptides as Inhibitors of Glutathionylspermidine Synthetase. Part 1: Substitution of the Glycine Carboxylic Acid Group. Bioorg. Med. Chem. Lett. 2002, 12, 2553-2556.

(42) Amssoms, K.; Oza, S. L.; Augustyns, K.; Yamani, A.; Lambeir, A. M.; Bal, G.; Van der Veken, P.; Fairlamb, A. H.; Haemers, A. Glutathione-like Tripeptides as Inhibitors of Glutathionylspermidine Synthetase. Part 2: Substitution of the Glycine Part. Bioorg. Med. Chem. Lett. 2002, 12, 2703-2705.

(43) D’Silva, C.; Daunes, S.; Rock, P.; Yardley, V.; Croft, S. L. StructureActivity Study on the In Vitro Antiprotozoal Activity of Glutathione Derivatives. J. Med. Chem. 2000, 43, 2072-2078.

(44) Daunes, S.; D’Silva, C.; Kendrick, H.; Yardley, V.; Croft, S. L. QSAR Study on the Contribution of $\log P$ and $E$ s to the in Vitro Antiprotozoal. Activity of Glutathione Derivatives. J. Med. Chem. 2001, 44, 2976-2983.

(45) Ravaschino, E. L.; Docampo, R.; Rodriguez, J. B. Synthesis and Biological Evaluation of Glutathione-like Tripeptides against Trypanosoma cruzi. Arkivoc 2003, Part (x), 298-313.

(46) Chen, S.; Lin, C.-H.; Kwon, D. S.; Walsh, C. T.; Coward, J. K. Design, Synthesis, and Biochemical Evaluation of Phosphonate and Phosphonamidate Analogues of Glutathionylspermidine as Inhibitors of Glutathionylspermidine Synthetase/Amidase from Escherichia coli. J. Med. Chem. 1997, 40, 3842-3850.

(47) Kwon, D. S.; Lin, C.-H.; Chen, S.; Coward, J. K.; Walsh, C. T.; Bollinger, J. M., Jr. Dissection of Glutathionylspermidine Synthetase/ Amidase from Escherichia coli into Autonomously Folding and Functional Synthetase and Amidase Domains. J. Biol. Chem. 1997, 272, 2429-2436

(48) Griffith, O. W.; Meister, A. Potent and Specific Inhibition of Glutathione Synthesis by Buthionine Sulfoximine ( $S$ - $n$-Butyl Homocysteine Sulfoximine). J. Biol. Chem. 1979, 254, 7558-7560.

(49) Boyd, E. A.; Regan, A, C.; James, K. Tetrahedron Lett. 1992, 33, 813-816. (c) Ösapay, G.; Szilagyi, I.; Seres, J. Tetrahedron 1987, 43, 2977-2983. (d) Hoffmann, M. Synthesis 1986, 557-558.

(50) Cinque, G. M.; Szajnman, S. H.; Zhong, L.; Docampo, R.; Rodriguez, J. B.; Gros, E. G. Structure-Activity Relationship of New Growth Inhibitors of Trypanosoma cruzi. J. Med. Chem. 1998, 41, 15401554.

(51) Szajnman, S. H.; Yan, W.; Bailey, B. N.; Docampo, R.; Elhalem, E.; Rodriguez, J. B.; Design and Synthesis of Aryloxyethyl Thiocyanate Derivatives as Potent Inhibitors of Trypanosoma cruzi Proliferation. J. Med. Chem. 2000, 43, 1826-1840.

(52) Elhalem, E.; Bailey, B. N.; Docampo, R.; Ujváry, I.; Szajnman, S. H.; Rodriguez, J. B. Design, Synthesis and Biological Evaluation of Aryloxyethyl Thiocyanate Derivatives against Trypanosoma cruzi. J. Med. Chem. 2002, 45, 3984-3999.

(53) Urbina, J. A.; Concepcion, J. L.; Montalvetti, A.; Rodriguez, J. B.; Docampo, R. Mechanism of action of 4-phenoxyphenoxy derivatives against Trypanosoma cruzi, the causative agent of Chagas disease. Antimicrob. Agents Chemother. 2003, 47, 2047-2050.

(54) Szajnman, S. H.; Bailey, B. N.; Docampo, R.; Rodriguez, J. B. Bisphosphonates Derived from Fatty Acids are Potent Growth Inhibitors of Trypanosoma cruzi. Bioorg. Med. Chem. Lett. 2001, 11, 789-792.

(55) Szajnman, S. H.; Montalvetti, A.; Wang, Y.; Docampo, R.; Rodriguez, J. B. Bisphosphonates Derived from Fatty Acids are Potent Inhibitors of Trypanosoma cruzi Farnesyl Pyrophosphate Synthase. Bioorg. Med. Chem. Lett. 2003, 13, 3231-3235.

JM050922I 